\title{
On the stability of massive stars
}

\author{
Dissertation \\ zur Erlangung des mathematisch-naturwissenschaftlichen Doktorgrades \\ "Doctor rerum naturalium" \\ der Georg-August-Universität Göttingen \\ im Promotionsprogramm PROPHYS \\ der Georg-August University School of Science (GAUSS)
}

vorgelegt von

Abhay Pratap Yadav

aus Ghazipur, Indien

Göttingen, 2016 
Betreuungsausschuss

Prof. Dr. Wolfgang Glatzel

Institut für Astrophysik, Georg-August-Universität, Göttingen, Deutschland

Prof. Dr. Wolfram Kollatschny

Institut für Astrophysik, Georg-August-Universität, Göttingen, Deutschland

Mitglieder der Prüfungskommision

Referent: Prof. Dr. Wolfgang Glatzel

Institut für Astrophysik, Georg-August-Universität, Göttingen, Deutschland

Korreferent: Prof. Dr. Wolfram Kollatschny

Institut für Astrophysik, Georg-August-Universität, Göttingen, Deutschland

Weitere Mitglieder der Prüfungskommission:

Prof. Dr. Stefan Dreizler

Institut für Astrophysik, Georg-August-Universität, Göttingen, Deutschland

Prof. Dr. Jens Niemeyer

Institut für Astrophysik, Georg-August-Universität, Göttingen, Deutschland

Prof. Dr. Martin Rein

Institut für Aerodynamik und Strömungstechnik, DLR, Göttingen, Deutschland

Prof. Dr. Karl-Henning Rehren

Institut für Theoretische Physik, Georg-August-Universität, Göttingen, Deutschland

Tag der mündlichen Prüfung: 11 Juli 2016 


\section{Abstract}

On the basis of the equations for stellar structure together with an equation of state stellar models may be constructed. Using these stellar models, the effects of different parameters on the evolution and internal structure of stars can be studied. The stability of stellar models is investigated by applying perturbations to the set of dependent variables. For infinitesimally small perturbations higher order terms can be neglected and a linear stability problem is obtained (linear approximation). The mathematical problem then poses a boundary eigenvalue problem with the complex eigenfrequencies of the stellar models considered as eigenvalues. The real parts correspond to the inverse of the pulsation periods whereas the imaginary parts indicate the growth rate of an unstable mode or damping of a damped mode respectively. Thus this approach provides an estimate of possible pulsation periods which may be compared with observed periods of a star with parameters close to that of the model considered. A linear approach can never predict the amplitude of an oscillation. Therefore nonlinear simulations are required to determine, e.g., the final velocity amplitude or the final variation of the brightness of the object. Moreover, the final period in the nonlinear regime might be different from the linear period due to nonlinear effects. In many models studied here it has been found that the linearly determined periods substantially differ from the periods obtained by nonlinear simulations. Therefore nonlinear simulations are inevitable if theoretically determined periods are to be compared with observed periods in stars.

In this thesis, linear stability analyses together with nonlinear simulations have been performed for a variety of models for massive stars. The linear pulsation equations are solved using the Riccati method which has the advantage that the frequencies and eigenfunctions of even high order modes can be calculated with prescribed high accuracy. In order to determine the final fate of unstable models, nonlinear simulations are performed. If these simulations are to be meaningful, they have to satisfy an extremely high accuracy, since the energies of interest (e.g., the kinetic energy) are by several orders of magnitude smaller than the dominant energies (gravitational potential and internal thermal energy). The requirements are met by the fully conservative numerical scheme adopted. Full conservativity is achieved by implicit time integration.

For the stability analysis envelope models for zero age main sequence stars with solar chemical composition in the mass range between $50 \mathrm{M}_{\odot}$ and $150 \mathrm{M}_{\odot}$ have been constructed. The linear stability analysis of these models reveals instabilities above $58 \mathrm{M}_{\odot}$. The pulsation periods of unstable modes lie in the range between 3 hours and 1 day. Nonlinear simulations of unstable models indicate that their final state is associated with pulsationally driven mass loss and mass loss rates of the order of $10^{-7}$ $\mathrm{M}_{\odot} / \mathrm{yr}$.

Recent observations of the B-type supergiant 55 Cygni, reveal that this star pulsates with periods in the range between 2.7 hours and 22.5 days. The authors identify the pulsations with pressure, gravity and strange modes. Motivated by the observations we have performed a linear stability analysis of corresponding stellar models together with nonlinear simulations of unstable models. As a result we find that the mass of 55 Cygni lies below $28 \mathrm{M}_{\odot}$. The pulsation periods derived from nonlinear simulations 
lie well within the range of observed periods and the mass loss estimated from the simulations is consistent with observed mass loss.

In the linear stability analysis of zero age main sequence models a set of non oscillatory (monotonically) unstable modes has been identified. Such modes are present both for radial and nonradial perturbations. Their growth rates vary with the harmonic degree and their kinetic energies show a secondary maximum very close to the surface of the models which may indicate the possibility of an observational identification. In the thesis, we present an attempt to understand the behaviour and origin of these modes. 


\section{Acknowledgements}

I would like to extend my sincere gratitude and indebtedness to my advisor Prof. Dr. Wolfgang Glatzel for his consistent support, and the knowledge he transferred right from the Master's in Astronomy and Astrophysics till today, the doctoral studies in Goettingen. I deeply appreciate your continuous availability and readiness to solve my queries. Your encouragement and motivation always directed me toward the right track. I am grateful to my co-advisor Prof. Dr. Wolfram Kollatschny for his valuable advice and guidance during my studies. I really admire your humble nature and willingness to help in every meeting.

I am grateful to my thesis committee members Prof. Dr. Stefan Dreizler, Prof. Dr. Martin Rein, Prof. Dr. Jens Niemeyer and Prof. Dr. Karl-Henning Rehren for providing their valuable time on a short notice. I thank to Dr. Frederic V. Hessman for his advice on the University guidelines for doctoral programs.

I gratefully acknowledge the financial support for pursuing doctoral studies, attending conferences and schools from European Union through EXPERTS III - Erasmus Mundus scholarship. I wholeheartedly express many thanks to all the EXPERTS III consortium members for providing me this wonderful opportunity to pursue the $\mathrm{PhD}$ project and follow my dreams. I highly appreciate the continuous support from the International Office of the University of Goettingen, specially EXPERTS team including Philippa, Netra, Agnieszka and Esther. I also thank to Dr. Vitali Altholz and Janja Mohorko for their valuable suggestions. My sincere thanks to the supporting staff at the Institute for Astrophysics for helping me at many academic and nonacademic fronts. I also acknowledge the assistance from Dr. Klaus Reinsch and Wilfried Steinhof to obtain computational resources.

My stay in the beautiful city of Goettingen would not have been so delightful without my friends and colleagues specially Flo, Luis, Sudeshna, Julian, Daniel, Elmira, Johannes, Chris, Niclas, Vera, Andreas, Laura, Martin and Avinash. Special thank goes to Chaitanya, Aishwarya, Gaurav and Gitanjali for making my weekends more enjoyable. I am thankful to Dr. Matthias Zetzl for helping me on computer related issues as well as enhancing my knowledge on German food during lunch hours. Interactions with you guys kept my spirits high and alive.

I also admire the support and encouragement from my friends outside Goettingen, particularly Kalyan, Kranthi, Cosmos and Jayanath. I thank my parents, siblings and family for their unconditional love and support. Last but not the least, I am grateful to my wife Rashmi and daughter Saanvi for their understanding and continuous support specially during thesis writing period.

I am what I am due to the support, sweat and shadow of you all. My sincere and heartfelt thanks to everyone of you. 



\section{Overview}

The thesis is divided into six chapters (for a visualization see Fig. 1). Chapter 1 presents an introduction to the topic and a motivation for the study. The basic equations and methods used are discussed in Chapter 2. The results obtained are discussed in the following three chapters. Chapter 3 focuses on the studies of massive main sequence stars. Motivated by recent observations theoretical calculations and simulations for models of the B-type supergiant 55 Cygni (HD 198478) are described in Chapter 4. Nonradial monotonically unstable modes in zero age main sequence models are the

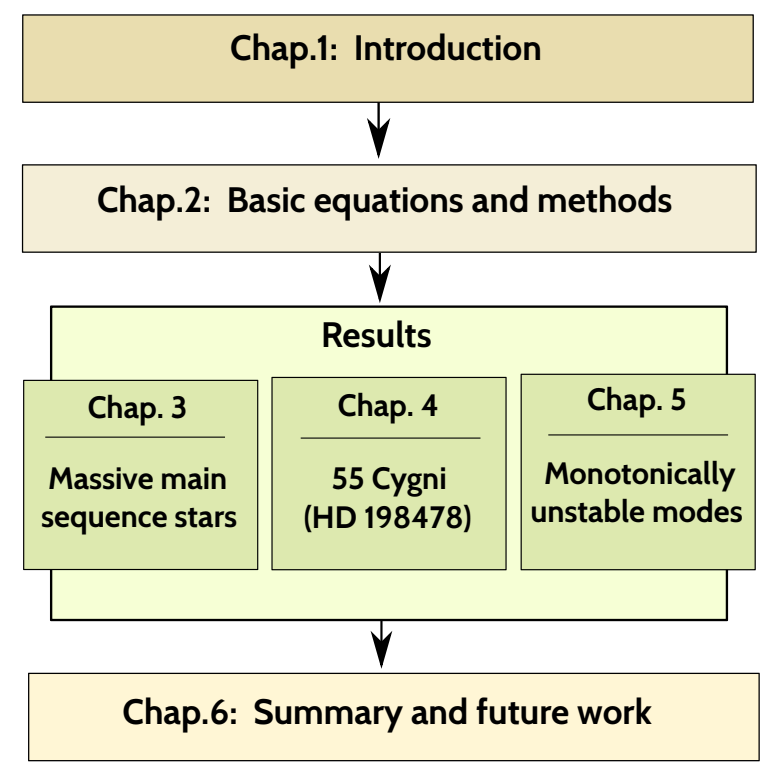

FIGURE 1: Outline of the thesis

subject of Chapter 5. Their properties as well as their dependence on the harmonic degree are discussed there. Finally, a summary and plans for future work are presented in Chapter 6. 



\section{Contents}

$\begin{array}{ll}\text { Abstract } & \text { iii }\end{array}$

Acknowledgements $\quad$ v

Overview vii

1 Introduction 1

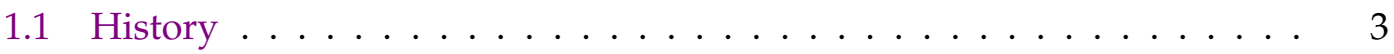

1.2 Objectives and motivation $\ldots \ldots \ldots \ldots \ldots$

2 Basic equations and methods $\quad 7$

2.1 Envelope models . . . . . . . . . . . . . . . . . . . 8

2.2 Linear stability analysis . . . . . . . . . . . . . . . . . . . . . . . . . . 10

2.3 Solution of the linear pulsation equations . . . . . . . . . . . . . . 12

2.3.1 The adiabatic approximation . . . . . . . . . . . . . 12

2.3.2 The Riccati method . . . . . . . . . . . . . . . . . . . . . . . 14

2.3 .3 Strange modes . . . . . . . . . . . . . . . . . 18

2.4 Nonlinear Simulation . . . . . . . . . . . . . . . . . . . . . 21

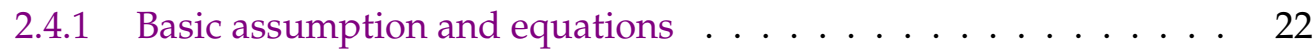

2.4 .2 Boundary conditions . . . . . . . . . . . . . . . . 24

2.4 .3 Numerical scheme . . . . . . . . . . . . . . . . . . . 25

2.4 .4 Validation of the scheme . . . . . . . . . . . . . 26

2.5 Nonradial perturbations . . . . . . . . . . . . . . . . . . . . . . 29

3 Massive main sequence stars 31

3.1 Stellar models . . . . . . . . . . . . . . . . . . . 32

3.2 Linear stability analysis . . . . . . . . . . . . . . . . 32

3.3 Results of the linear stability analysis . . . . . . . . . . . . . . . 33

3.4 Nonlinear simulations . . . . . . . . . . . . . . . . . . . . 34

3.5 Results of nonlinear simulations for selected stellar models . . . . . . . . 35

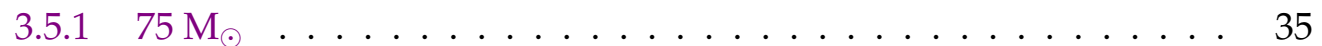

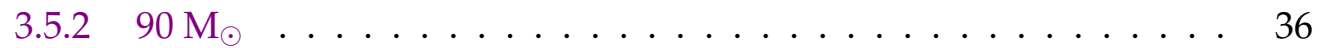

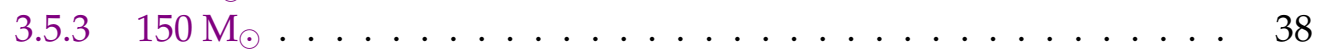

3.6 Discussion and conclusions . . . . . . . . . . . . . . 39

455 Cygni (HD 198478) 41

4.1 Introduction . . . . . . . . . . . . . . . . . 41

4.2 Models . . . . . . . . . . . . . . . . . . . . . . 42

4.3 Linear stability analysis . . . . . . . . . . . . . . . . . . 42

4.4 Results of the linear stability analysis . . . . . . . . . . . . . . . . . 43

4.5 Nonlinear simulations . . . . . . . . . . . . . . . . . . . . 45

4.5.1 Models with solar composition . . . . . . . . . . . . . . . 46

$23 \mathrm{M}_{\odot} \ldots \ldots \ldots \ldots \ldots \ldots$ 
$25 \mathrm{M}_{\odot} \ldots \ldots \ldots \ldots \ldots \ldots \ldots \ldots$

4.5.2 Models with enhanced He abundance . . . . . . . . . . . . . . 48

4.6 Discussion and conclusions . . . . . . . . . . . . . . 52

5 Monotonically unstable modes in main sequence stars $\quad 55$

5.1 Introduction . . . . . . . . . . . . . . . . . . . 55

5.2 Stellar Models . . . . . . . . . . . . . . . . . . . . . . 55

5.3 Stability Analysis . . . . . . . . . . . . . . . . . 57

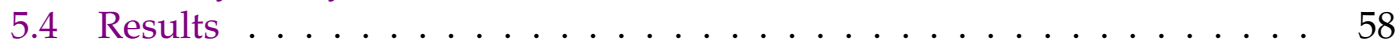

5.4.1 Radial and non-radial monotonically unstable modes . . . . . . . 58

5.4.2 Dependence on harmonic degree $(l)$ of monotonically unstable modes ............................ 58

5.4.3 Distribution of kinetic energies of monotonically unstable modes 58

5.5 Discussion and conclusions . . . . . . . . . . . . . . . . . . 60

6 Summary and future work $\quad 63$

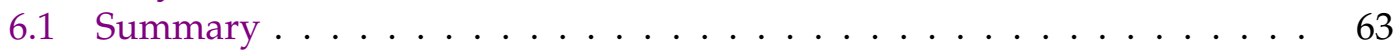

6.1 .1 Main sequence stars . . . . . . . . . . . . . . . 63

6.1 .255 Cygni (HD 198478) . . . . . . . . . . . . . . . . . . 63

6.1 .3 Monotonically unstable modes . . . . . . . . . . . . . . . . . . 64

6.2 Future work . . . . . . . . . . . . . . . . . . . 64

$\begin{array}{ll}\text { Curriculum vitae } & 73\end{array}$ 


\section{List of Figures}

1 Outline of the thesis . . . . . . . . . . . . . vii

1.1 Instability induced finite amplitude variation of stellar radius (a) and surface temperature $(b)$ of a $90 \mathrm{M}_{\odot}$ ZAMS model having solar chemical composition. . . . . . . . . . . . . . . . .

2.1 Core-envelope structure of a massive star (a) and the boundaries of envelope models $(b) . \ldots \ldots$. . . . . . . . . . . . . . 9

2.2 Integration strategy for the construction of envelope models. . . . . . . . 10

2.3 The ratio of local thermal and dynamical timescales as a function of the relative radius $(x)$ for two different stellar models corresponding to a Cepheid and a HdC star, adopted from Gautschy \& Glatzel (1990b). . . .

2.4 Sketch of the integration strategy for the integration of the Riccati equations. . . . . . . . . . . . . . . . . . 16

2.5 The Riccati determinant as a function of the real part $\left(\sigma_{\mathrm{r}}\right)$ of the eigenfrequency. . . . . . . . . . . . . . . . . . 16

2.6 A cartoon representation of a modal diagram. . . . . . . . . . . . . 17

2.7 Real $\left(\xi_{\mathrm{r}}\right)$ and imaginary $\left(\xi_{\mathrm{i}}\right)$ parts of the Lagrangian displacement for a damped high order p-mode. . . . . . . . . . . . . . . . 18

2.8 Modal diagram for models of Wolf-Rayet stars adopted from Glatzel et al. (1993). . . . . . . . . . . . . . . . . . . . 19

2.9 Same as Fig. 2.8 but within the NAR approximation. . . . . . . . . . . . 20

2.10 Ratio of convective and total luminosity $\left(\mathrm{L}_{\mathrm{con}} / \mathrm{L}_{\text {total }}\right)$ as a function of relative radius. . . . . . . . . . . . . . . . . . 23

2.11 Propagation of shock waves near the outer boundary in a model for 55 Cygni. . . . . . . . . . . . . . . . . . 24

2.12 Definition of variables on the staggered grid. . . . . . . . . . . . . . 25

2.13 Snapshots of the density stratification as a function of normalized radius. 26

2.14 Simulation of the evolution of an instability for a $90 \mathrm{M}_{\odot}$ ZAMS model. . 27

2.15 Photospheric velocity as a function of time for two stable ZAMS models. 28

3.1 Real (a) and imaginary parts (b) of eigenfrequencies normalized by the global free fall time as a function of mass along a ZAMS with solar chemical composition. Unstable modes are represented by thick lines in (a) and by negative values of the imaginary part in (b). . . . . . . . . .

3.2 Same as Fig. 3.1 but for photospheric boundary conditions consistent with the previous study. . . . . . . . . . . . . . 34

3.3 Evolution of instabilities and finite amplitude pulsations of a ZAMS model

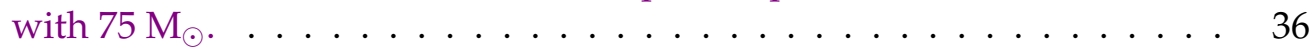

3.4 Same as Fig. 3.3 but for a ZAMS model with $90 \mathrm{M}_{\odot} \ldots \ldots$. . . . . . . . . . . 37

3.5 Same as Fig. 3.3 but for a ZAMS model with $150 \mathrm{M}_{\odot}$. . . . . . . . . . . 38 
4.1 Real (a) and imaginary (b) parts of the eigenfrequencies normalized to free fall time as a function of mass for models of 55 Cygni with luminosity $\log L / L_{\odot}=5.57$, effective temperature $18800 \mathrm{~K}$ and solar chemical composition ( $\mathrm{X}=0.70, \mathrm{Y}=0.28, \mathrm{Z}=0.02$ ). Thick lines in (a) and negative imaginary parts in (b) correspond to unstable modes. . . . . . . . . . . .

4.2 Same as Fig. 4.1 but for enhanced He abundance $(Y=0.78, X=0.20)$. . .

4.3 Same as Fig. 4.1 but for different outer boundary conditions . . . . . . .

4.4 Same as Fig. 4.3 but for enhanced He abundance $(Y=0.78, X=0.20)$. . .

4.5 The evolution of instabilities in a $23 \mathrm{M}_{\odot}$ model with solar chemical com-

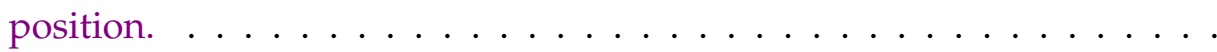

4.6 Same as Fig. 4.5 but for a model with $25 \mathrm{M}_{\odot} \ldots \ldots \ldots$. . . . . . . . . .

4.7 The evolution of a $34 \mathrm{M}_{\odot}$ model with enhanced He abundance $(X=0.20$, $Y=0.78)$. The following quantities are given as a function of time: Radius (a), velocity (b) and temperature (c), density (e) and pressure (f) of the outermost grid point, the variation of the bolometric magnitude in

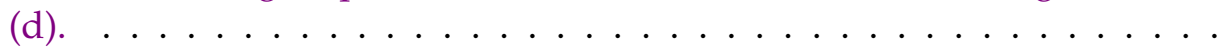

4.8 Same as Fig. 4.7 but for $30 \mathrm{M}_{\odot} \quad \ldots \ldots \ldots \ldots \ldots$. . . . . . . . .

4.9 The evolution of instabilities in a $17 \mathrm{M}_{\odot}$ model with enhanced He abundance. . . . . . . . . . . . . . . . . . . .

4.10 Density as a function of relative radius for the $25 \mathrm{M}_{\odot}$ model (dashed line) and the $17 \mathrm{M}_{\odot}$ model (full line) of 55 Cygni and for a model of the LBV AG Car (dotted line). . . . . . . . . . . . . . . . . . . .

5.1 Zero age main sequences in the HRD for three different metallicities. . .

5.2 Convection zones $\left(\nabla>\nabla_{a d}\right)$ of three ZAMS models with metallicity $\mathrm{Z}=$

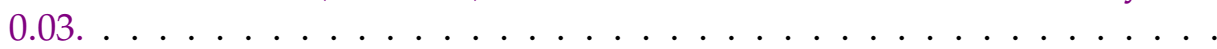

5.3 Growth rates (normalized by the global free fall time) of unstable nonradial modes with $l=2$ as a function of mass for ZAMS models with metallicity $\mathrm{Z}=0.03$ (left panel) and $\mathrm{Z}=0.02$ (right panel) . . . . . . . . .

5.4 Real and imaginary parts of the eigenfrequencies (normalized by the global free fall time) of unstable modes associated with the monotonically unstable modes $\mathrm{mu}_{1}$ and $\mathrm{mu}_{2}$ as a function of harmonic degree $(l)$ for three ZAMS models with metallicity $\mathrm{Z}=0.03 \ldots \ldots \ldots \ldots$. . . . .

5.5 Normalized kinetic energy as a function of relative radius of the two monotonically unstable modes $\mathrm{mu}_{1}$ and $\mathrm{mu}_{2}$ with $l=2$ for two ZAMS models having the metallicity $\mathrm{Z}=0.03 . \ldots \ldots \ldots$

5.6 Same as Fig. 5.5 but for a ZAMS model with $50 \mathrm{M}_{\odot} \ldots$. . . . . . . . . . .

5.7 Ratio of the radiative and the Eddington luminosity as a function of relative radius for three ZAMS models with metallicity $\mathrm{Z}=0.03 . \ldots .$.

6.1 Nonlinear evolution of the instability of a chemically peculiar (enhanced helium) model for HD 50064 having $\mathrm{T}_{\text {eff }}=13500 \mathrm{~K}, \log \left(\mathrm{L} / \mathrm{L}_{\odot}\right)=6.1$ and $\mathrm{M}=55 \mathrm{M}_{\odot} \ldots \ldots \ldots \ldots \ldots \ldots \ldots \ldots \ldots$

6.2 Modal diagram for models of $\zeta$ Pup with enhanced He abundance ( $\mathrm{Y}=$ 0.58 and $\mathrm{Z}=0.02) \ldots \ldots \ldots \ldots \ldots \ldots$

6.3 Nonlinear evolution of instabilities of a model for $\zeta$ Pup with $\mathrm{M}=44 \mathrm{M}_{\odot}$. 


\section{List of Tables}

2.1 List of variables and their physical meaning. . . . . . . . . . . . . . 13

3.1 Mass, effective temperature and luminosity on the zero age main sequence for solar chemical composition. . . . . . . . . . . . . 33

3.2 Pulsation periods and mass loss rates for the ZAMS models selected. . . 39 



\section{Chapter 1}

\section{Introduction}

Stars are basic constituents of the universe and by definition undergo nuclear burning at least once in their life. Theoretical calculations supported by corresponding observations exhibit a lower mass limit of $0.08 \mathrm{M}_{\odot}$ for a star to be able to ignite nuclear burning (see e.g., Dantona \& Mazzitelli, 1985). This lower limit is sensitive to the chemical composition and decreases with increasing metallicity. The evolution of such low mass stars proceeds on very long time scales (comparable to the Hubble time scale). Therefore an observational verification of the theoretically determined evolution of low mass stars is not possible. On the other hand, the evolution of massive stars proceeds on comparatively short time scales (depending on mass as short as $10^{6} \mathrm{yrs}$ ). Whether an upper limit for the mass of stars exits is still a matter of debate and depends on the physical process considered which might infer an upper mass limit. One of these processes involves the stability of a star. Early studies on the stability of main sequence stars have revealed upper (stability) mass limits of $100 \mathrm{M}_{\odot}$ (Ledoux, 1941) and $60 \mathrm{M}_{\odot}$ (Schwarzschild \& Härm, 1959).

Massive stars seem to play a crucial role in the chemical enrichment of galaxies (Nomoto et al., 2013) and the most massive primordial stars are likely to be main sources of radiation in the early universe (re-ionization). Tanvir et al. (2009) reported a Gamma Ray Burst (GRB) at a redshift of 8.2 and interpreted its occurrence as an indication that 'massive stars were being produced and dying' in the early universe approximately $630 \mathrm{Myr}$ after the Big Bang. The occurrence of a GRB at even higher redshift (Cucchiara et al., 2011) strengthens this idea.

In the previous decades many authors (see e.g., Figer, 2005; Weidner \& Kroupa, 2004) claimed the existence of an upper mass limit for stars of around $150 \mathrm{M}_{\odot}$. However, Crowther et al. (2010) reported the existence of stars in the star cluster R136 with masses above $150 \mathrm{M}_{\odot}$. This star cluster is situated in 30 Doradus, an H II region of the Large Magellanic Cloud. These studies show that the star cluster R136 is a region likely to harbor the most massive stars. Hence it is a field of interest for existing as well as next generation telescopes. Particularly interesting is the star R136a1 whose mass is suggested to lie above $300 \mathrm{M}_{\odot}$ (see, Crowther et al., 2016).

According to our present understanding of stellar evolution, stars having masses above than approximately $8 \mathrm{M}_{\odot}$ end their life with a supernova explosion. The final product is then either a neutron star or a stellar black hole depending on the initial mass of the star. However, the post main sequence evolution of massive stars severely depends on stellar mass loss whose origin, mechanism and magnitude are largely unknown. Thus our lack of knowledge concerning mass loss introduces an ambiguity to predict the final fate of massive stars. Therefore a reliable theory of mass loss is desperately needed to understand the evolution of massive stars. Despite several attempts in this direction (see e.g., Vink et al., 2001) a proper understanding of mass loss in massive stars is still missing. Stellar winds, pulsations, eruptions of surface layers from 
supergiants and binary mass transfer are the main processes discussed for the mass loss in massive stars (Smith, 2014). Photometric and spectroscopic variability in many massive stars revealed the presence of pulsations in these objects. Meanwhile there is growing evidence for a connection between mass loss and pulsation in massive stars. For example, Kraus et al. (2015) suggested that the pulsation observed in the B-type supergiant 55 Cygni can trigger enhanced phases of mass loss.

In an early observational study of very massive stars Humphreys \& Davidson (1979) have identified an essentially empty region in the Hertzsprung - Russell diagram (HRD), where no stationary stellar object is found. This domain is confined by the HumphreysDavidson limit (Humphreys \& Davidson, 1979). The same authors suggested that the existence of this limit might be due to an instability which induces violent mass loss in stars. Rayleigh-Taylor instability as a result of density inversions occurring in corresponding stellar models and an associated turbulent pressure (de Jager, 1980, 1984) or a modified Eddington limit (Davidson, 1987; Humphreys \& Davidson, 1984; Lamers, 1986) were proposed as an explanation for the existence of the Humphreys-Davidson limit. In spite of all these efforts the origin of this limit is not yet fully understood. Glatzel \& Kiriakidis (1993b) reported on violent mode coupling instabilities (strange mode instabilities) in models for Luminous Blue Variables (LBVs). The stability boundary for these instabilities in the HRD coincides with the Humphreys-Davidson limit. Strange mode instabilities have a non thermal origin which can be proven using the Non Adiabatic Reversible (NAR) approximation (Gautschy \& Glatzel, 1990b). The existence of strange mode instabilities even in the NAR approximation proves them to be of acoustic origin. Meanwhile the presence of strange mode instabilities has been reported in a variety of stellar models (see, e.g. Gautschy \& Glatzel, 1990b; Jeffery \& Saio, 2016; Saio \& Jeffery, 1988; Wood, 1976) including the zero age main sequence (ZAMS). The growth rates associated with these instabilities are much higher compared to those of $\kappa$ and $\epsilon$ - mechanism as mentioned by Glatzel \& Kiriakidis (1993a). Moreover, these authors have also emphasized that the instability associated with fundamental mode in massive ZAMS models is extremely weak and its growth time scale competes with the nuclear and evolution time scale. A study dedicated to the fundamental mode in models of massive main sequence stars was presented by Goodman \& White (2016). These authors also concluded that the radial fundamental mode exhibits a small growth rate and the instability associated with this mode would not limit the main sequence life time.
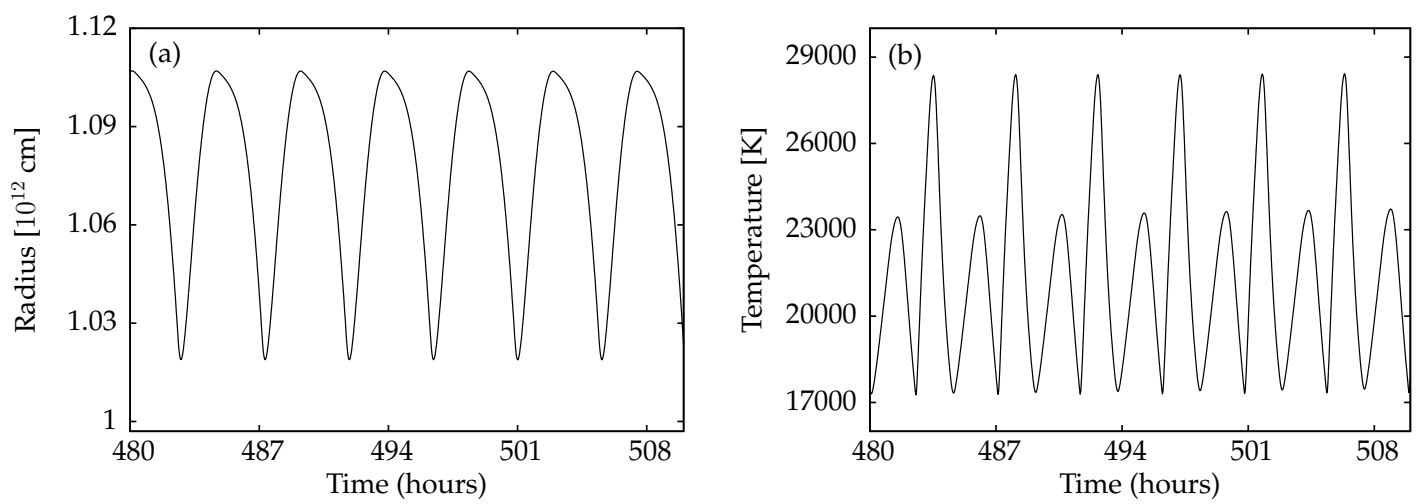

FIGURE 1.1: Instability induced finite amplitude variation of stellar radius (a) and surface temperature (b) of a $90 \mathrm{M}_{\odot}$ ZAMS model having solar chemical composition. 
The solution of the linear pulsation equations together with appropriate boundary conditions forms a boundary eigenvalue problem is generally referred to as 'linear stability analysis'. The linear stability analysis provides the information, whether a given stellar model is unstable or stable with respect to infinitesimally small perturbations. For a given model several modes may be unstable simultaneously due to various mechanisms.

The final fate of a linearly unstable stellar model, however, can only be determined by following the instability into the nonlinear regime. In most cases the nonlinear simulations show, that instabilities lead to periodic pulsations, where the period can easily be deduced from the variations of the stellar parameters (e.g., radius or effective temperature) as a function of time in the model considered. For example, Fig. 1.1 illustrates the final finite amplitude pulsation in terms of a strict periodic variation of radius and surface temperature of a linearly unstable zero age main sequence model with solar chemical composition and a mass of $90 \mathrm{M}_{\odot}$. Apart from strictly periodic pulsations instabilities may also lead to irregular pulsations.

One of the most interesting consequences of violent instabilities is the possibility that they may induce direct mass loss. Should the velocity amplitude in the nonlinear regime of the evolution of an instability exceed the escape velocity from the object, this is taken as an indication of direct mass loss. In fact, this phenomenon has been found in simulations of models for massive stars (Glatzel et al., 1999) and we will report on it in this thesis for a model of 55 Cygni (see Chap.4).

\subsection{History}

Pulsations in stars have been observed as early as 1786 in the case of the variable star $\delta$-Cephei (Goodricke \& Bayer, 1786). In the beginning, periodic stellar variability was thought to be caused by a binary system.

Technical improvements of the telescopes helped to discover a large number of variable stars. Shapley (1914) suggested the idea of radial pulsations as a cause of variability in cepheids. With the help of a huge number of observations Leavitt (1908) discovered the existence of a period- luminosity relation, which was improved by Hertzsprung (1914) later on and has been used to determine the distance to the Small Magellanic Cloud (see also Smolec, 2009). The period-luminosity relation provides a unique way to measure distances within the Milky Way and even distances to other galaxies with the help of pulsating stars. According to Christensen-Dalsgaard (2014), the understanding of pulsation mechanisms and the cause of pulsations are among the main reasons to study stellar pulsations. Pulsation modes in a star may provide unique information about the internal structure of a star. Actually deriving the internal properties of a star with the help of observed pulsation frequencies is the primary goal of asteroseismology (see also Aerts et al., 2010a). As an example for this technique, back in 1879, Ritter (1879) reported the existence of a period-density relation. This relation immediately provides an estimate of the density of the star once an observed period is identified with the pulsation period of a mode.

The mechanisms exciting pulsations remained unclear until Eddington (1926) considered pulsating stars as heat engines where suitable conditions can lead to self excited pulsations. Apart from a modulation of the energy generation by nuclear sources 
in the stellar core, he also proposed a valve mechanism (today addressed as the $\kappa$ mechanism) to excite pulsations. The latter relies on the dependence of the heat transport on temperature and density (by means of the opacity) and is independent of nuclear energy generation in the core (see Gautschy, 1997, for an extensive review, in particular for the historical developments).

Epstein (1950) showed that in the stellar core the perturbations associated with a pulsation become very small compared to the amplitude of the perturbations at the stellar surface (with a ratio of approximately $10^{-6}$ ). This clearly indicates that the stellar core is not severely affected by pulsations. As a result, for many cases the stellar core (and, in particular, nuclear reactions) can safely be ignored in pulsation studies. However, in order to investigate the excitation of pulsations by nuclear reactions $(\varepsilon-$ mechanism) the stellar core must be taken into account. The fact that the stellar core can be ignored when considering stellar pulsations is also reflected in the behaviour of eigenfunctions obtained in a linear stability analysis. Eigenfunctions decrease exponentially from the surface to the center of stellar models (see Fig. 2.7). Disregarding helium and hydrogen ionization in models of red giant stars, Cox (1955) found pulsations to be damped on a timescale of 10 days. On the other hand, Zhevakin in 1953 found pulsations to be excited when fully including the effect of He II zones in his models (Zhevakin, 1963). Detailed numerical calculations for models of $\delta$-Cephei were performed by Baker \& Kippenhahn (1962). These authors confirmed the important role of ionization zones for the driving of stellar pulsations. In this thesis, the linear pulsation equations will be considered in a form (with some modifications) similar to that given by Baker \& Kippenhahn (1962). The excitation of pulsations observed in $\beta$-Cepheid stars was a mystery for a long time and found its solution when a significant contribution of heavy elements to the opacity was discovered (Iglesias \& Rogers, 1996; Rogers \& Iglesias, 1992; Rogers et al., 1996). The peak in the opacity due to heavy elements (in particular Fe-group elements) around $\mathrm{T}=200,000 \mathrm{~K}$ is generally referred to as the Fe-opacity bump. Numerical studies based on these improved opacities for models of $\beta$-Cepheids (Dziembowski \& Pamiatnykh, 1993; Kiriakidis et al., 1992) revealed that the $\kappa$-mechanism associated with the Fe-opacity bump is responsible for the excitation of pulsations in these stars.

\subsection{Objectives and motivation}

For a wide range of parameters massive stars, even massive ZAMS stars have been found to be violently unstable due to strange mode instabilities (Glatzel \& Kiriakidis, 1993a). These studies are based on linear stability analyses. The aim of the present thesis is to perform a linear stability analysis for models of massive stars thus confirming previous studies and, as an extension, to follow the instabilities of unstable modes into the nonlinear regime. In general the final fate of unstable models can only be determined by following the instabilities into the nonlinear regime. Extensive nonlinear simulations have not been done so far and are further motivated by two issues: As mentioned by some authors (see, e.g., Glatzel, 2009) pulsation periods obtained by linear stability analyses do not match the observed period in several cases. In this thesis it will be of particular interest whether the linear pulsation periods are affected in the nonlinear regime of the evolution of a strong (strange mode) instability and nonlinear periods should be compared to the observed values rather than their linearly determined counterparts. The second question to be addressed is whether the strong strange mode instabilities might be responsible for stellar mass loss in massive stars. 
In general, the coupling between stellar pulsation and mass loss is poorly understood. Meanwhile there is growing evidence for a relation between mass loss and pulsation (see, e.g., Kraus et al., 2015). Mass loss severely affects the evolution and the final fate of massive stars (Smith, 2014). This is a further motivation for the present study of mass loss due to strange mode instabilities and pulsations. Estimates of mass loss rates will therefore will be of particular interest.

Following instabilities into the nonlinear regime faces several problems, one of which concerns the energy balance of the system. The fact that different forms of the energy differ by several orders of magnitude requires a sophisticated conservative numerical scheme which satisfies the energy balance intrinsically. Moreover, in the nonlinear regime shock waves are expected to be generated. They require special treatment.

A recent observational study by Kraus et al. (2015) suggests the presence of various pulsation modes including strange modes in the B-type supergiant 55 Cygni. In this thesis, a stability analysis and nonlinear simulations for stellar models with parameters close to that of 55 Cygni will be performed.

In models of massive stars, a new kind of non oscillatory modes with vanishing frequency has been found by Hilker (2009), Deller (2009) and Saio (2011). These modes exhibit strong growth rates in the dynamical range. Their appearance and consequences are not yet understood. In the present study an attempt will be made to study them systematically in main sequence models. 



\section{Chapter 2}

\section{Basic equations and methods}

To understand the physics and the evolution of stars a theoretical approach is inevitable. Due to the timescale involved in stellar evolution observations provide only snapshots of the evolution which need to be ordered and connected on the basis of a theoretical framework. Moreover, the interior of stars is not directly accessible by observations. Thus a theoretical description of the interior of stars is particularly important.

Stars are extremely complex systems. To enable a theoretical treatment, approximations and simplifications have to be introduced. The basic assumptions of the common first order approach to stellar structure and evolution may be summarized as follows (see, e.g., Kippenhahn et al., 2012; Salaris \& Cassisi, 2006):

- Stars consist of matter and radiation.

- To first order, rotation, magnetic fields, rotational mixing and atomic diffusion are neglected.

- Neglecting rotation and magnetic fields, stars can be described as one-dimensional spherically symmetric systems.

Within this simplified approach the equations governing stellar structure and evolution may be written in terms of the Lagrangian mass coordinate $m$ (the mass contained within a sphere of radius $r$ ) and the time $t$ as independent variables:

$$
\begin{gathered}
\frac{\partial r}{\partial m}=\frac{1}{4 \pi r^{2} \rho} \\
\frac{\partial P}{\partial m}=-\frac{G m}{4 \pi r^{4}}-\frac{1}{4 \pi r^{2}} \frac{\partial^{2} r}{\partial t^{2}} \\
\frac{\partial L}{\partial m}=\epsilon-C_{p} \frac{\partial T}{\partial t}+\frac{\delta}{\rho} \frac{\partial P}{\partial t} \\
\frac{\partial T}{\partial m}=-\frac{G m}{4 \pi r^{4}} \nabla \frac{T}{P}
\end{gathered}
$$

$\nabla$ characterizes the heat transport and is given by its radiative value $\nabla=\nabla_{\text {rad }}=$ $\frac{3 \kappa P}{16 \pi a c G} \frac{L_{\text {rad }}}{m T^{4}}$ if energy transport is entirely due to radiative diffusion. $\kappa$ denotes the opacity, $L_{\text {rad }}$ stands for the radiative luminosity, $a$ is the radiation constant, $c$ the speed of light, $T$ denotes the temperature and $P$ is the pressure. Within the Lagrangian description the position of a mass element in terms of its radius $r$ is a dependent variable. In the set of equations given above $L, G, \rho, \varepsilon, C_{p}$ and $\delta=\left.\left(\frac{d \log \rho}{d \log T}\right)\right|_{p}$ denote the total luminosity, the gravitational constant, the density, the nuclear energy generation rate, the specific heat at constant pressure and the thermal expansion coefficient, respectively. 
Equation 2.1 corresponds to mass conservation and equation 2.2 describes momentum conservation. Except for very short dynamical phases a star remains in hydrostatic equilibrium during stellar evolution. In hydrostatic equilibrium the acceleration term $\left(\frac{\partial^{2} r}{\partial t^{2}}\right)$ vanishes in equation 2.2. Energy conservation is expressed in terms of equation 2.3 and energy transport within a diffusion type approximation is described by equation 2.4. The equation governing the change of chemical composition due to nuclear reactions was not explicitly given here since chemical stellar evolution and nuclear reactions are disregarded in this thesis. (The timescales of these processes are much longer than the timescales considered here.)

To close the system of equations, an equation of state (EOS) has to be supplemented. The EOS in general provide a relation between pressure $(P)$, temperature $(T)$ and density $(\rho)$. For a mixture of an ideal gas and radiation, the pressure can be expressed as:

$$
P=\frac{a}{3} T^{4}+\frac{\Re}{\mu} \rho T
$$

where $a$ is the radiation constant, $\Re$ denotes the gas constant, and $\mu$ stands for the mean molecular weight. The first term in equation 2.5 represents the radiation pressure and the second term stands for the gas pressure.

The opacity describes the absorption of photons by the stellar matter. It plays an important role in any phase of stellar evolution. In the optically thick stellar interior the frequency dependence of the radiation field may be ignored. In this case the Rosseland mean $\kappa_{\text {rad }}$ of the opacity can be used to describe the transport of radiation within the diffusion approximation:

$$
\kappa_{\text {rad }}=\frac{\int_{0}^{\infty} \kappa_{\nu} F_{\nu} d \nu}{\int_{0}^{\infty} F_{\nu} d \nu}
$$

$\kappa_{\nu}$ is the monochromatic opacity and $F_{\nu}$ is the monochromatic flux at the frequency $\nu$. For local thermodynamic equilibrium, $F_{\nu}$ can be expressed in terms of the Planck function $B_{\nu}(T)$. The Rosseland mean of the opacity is then given by:

$$
\kappa_{\text {rad }}=\frac{\int_{0}^{\infty} \frac{d B_{\nu}(T)}{d T} d \nu}{\int_{0}^{\infty} \frac{1}{\kappa_{\nu}} \frac{d B_{\nu}(T)}{d T} d \nu} .
$$

If energy is transported both by radiation diffusion and conduction, the total opacity $\kappa$ is given by the harmonic mean of the radiative opacity $\left(\kappa_{r a d}\right)$ and the conductive opacity $\left(\kappa_{e}\right)$ :

$$
\frac{1}{\kappa}=\frac{1}{\kappa_{\text {rad }}}+\frac{1}{\kappa_{e}}
$$

The calculation of opacities for stellar matter is a challenging task. For convenience, opacities for astrophysical applications are usually provided in the form of tables covering a large range of densities, temperatures and chemical compositions (see, e.g., Cassisi et al., 2007; Mendoza et al., 2007). The OPAL opacity tables (Iglesias \& Rogers, 1996; Rogers \& Iglesias, 1992; Rogers et al., 1996) have been used for the present study.

\subsection{Envelope models}

Models for massive stars often exhibit a core-envelope structure, where the core with negligible radius contains almost the entire mass of the star, and the envelope with 


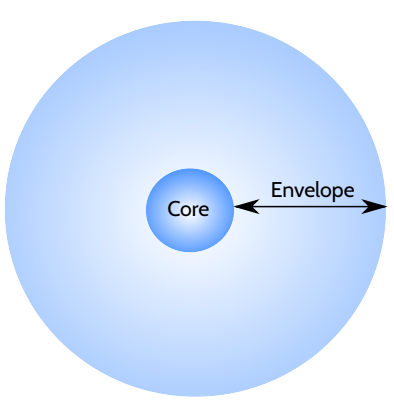

(a)

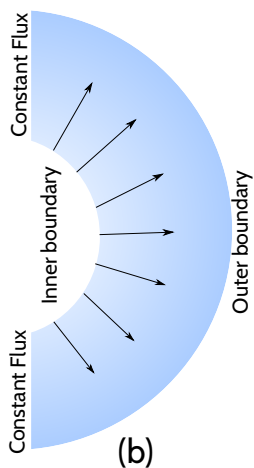

(b)

FIGURE 2.1: Core-envelope structure of a massive star (a) and the boundaries of envelope models (b).

negligible mass covers almost the entire stellar volume. The nuclear energy production takes place in the core, whereas the stellar envelope does not contain any sources or sinks of energy. Hence the luminosity is constant throughout the envelope. The latter considerably simplifies the construction of envelope models. Epstein (1950) pointed out that pulsation amplitudes exponentially decrease from the stellar surface to the center. Therefore the envelope of a stellar model plays the dominant role when considering stellar pulsations, whereas the stellar core may be disregarded. Thus investigations of stellar pulsations can be restricted to considering the stellar envelope only. Accordingly, the present study is based on envelope models for massive stars. Envelope models in hydrostatic equilibrium can be constructed by initial value integration once the effective temperature $\left(T_{e f f}\right)$, the luminosity $(L)$, the mass $M$ and the chemical composition are prescribed (see also, Grott, 2003). Note that the equations of stellar structure in general form a much more difficult boundary value problem. For an envelope with constant luminosity in hydrostatic and thermal equilibrium the stellar structure equations reduce to (see also, Grott, 2003):

$$
\begin{gathered}
\frac{\partial r}{\partial m}=\frac{1}{4 \pi r^{2} \rho} \\
\frac{\partial P}{\partial m}=-\frac{G m}{4 \pi r^{4}} \\
\frac{\partial T}{\partial m}=-\frac{G m}{4 \pi r^{4}} \nabla \frac{T}{P}
\end{gathered}
$$

$\nabla$ is evaluated on the basis of the mixing length theory (Böhm-Vitense, 1958). Equations $2.9-2.11$ are integrated as an initial value problem from the photosphere with radius $R$ to the inner boundary of the envelope by imposing the following three initial conditions at the photosphere $(m=M)$ :

1. $r=R$ is determined using Stefan-Boltzmann's law: $\mathrm{L}=4 \pi R^{2} \sigma_{B} T_{\text {eff }}^{4}$

2. Photospheric pressure $P=p_{\text {eff }}=\frac{1}{\kappa_{\text {eff }}} \frac{2 G M}{3 R^{2}}$

3. $\mathrm{T}=\mathrm{T}_{\mathrm{eff}}$

Once the mass $M$, the effective temperature $\mathrm{T}_{\text {eff }}$ and the luminosity $L$ together with a uniform chemical composition are specified, the initial conditions are determined without ambiguity. In the boundary conditions, $\sigma_{B}$ denotes Stefan-Boltzmann's constant. 
$p_{\text {eff }}$ and $\kappa_{\text {eff }}$ are the pressure and the opacity at the photosphere, respectively. Together with the boundary conditions given the set of differential equations (Eqs. 2.9 - 2.11) form an initial value problem to be integrated from the photosphere up to some maximum temperature (for a schematic representation of the integration strategy see Fig. 2.2). For the numerical integration any standard scheme may be used. In this thesis we have used a forth order implicit predictor corrector method.

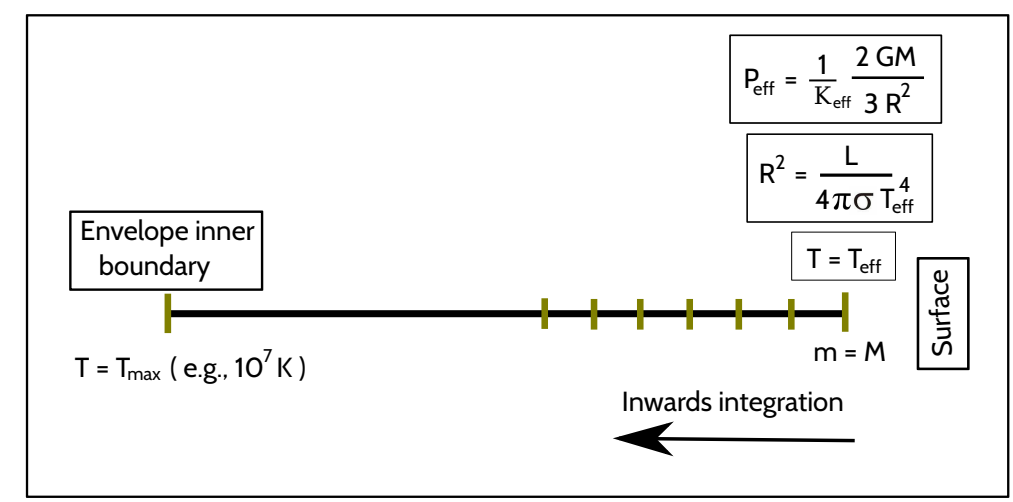

FIGURE 2.2: Integration strategy for the construction of envelope models.

\subsection{Linear stability analysis}

An approach to investigate the stability of a system consists of subjecting it to small perturbations. If a perturbation grows with time, the system is (linearly) unstable, if it decays, the system is (linearly) stable. As a first step, this approach is also applied here to stellar models. It has been adopted by many authors so far and is described, e.g., in the textbook on stellar pulsation by Cox (1980). In this thesis, we shall adopt the representation of Baker \& Kippenhahn (1962). These authors considered the stability of stellar models with respect to radial perturbations on the basis of the stellar structure equations (Eqs. 2.1 - 2.4): The dependent variables are decomposed into a stationary part satisfying hydrostatic and thermal equilibrium (which is assumed to be predetermined by envelope construction and referred to as background model) and a time dependent perturbation. Inserting this approach into Eqs. 2.1 - 2.4, assuming hydrostatic and thermal equilibrium to hold for the stationary parts and neglecting quadratic and higher order terms in the perturbations leads to a system of linear partial differential equations for the perturbations, where $m$ and $t$ are the independent variables. The time dependence can be separated by assuming an exponential time dependence of the perturbations of the form $\exp (i \omega t)$ where $\omega$ plays the role of a complex eigenfrequency. The partial differential equations for the perturbations then reduce to a set of ordinary differential equations (with $m$ as the independent variable) with the coefficients depending on the background model and the eigenfrequency. The latter will be referred to as the perturbation equations. Thus the stability problem is reduced to a fourth order system of ordinary differential equations which together with four suitable boundary conditions, to be discussed in the following, forms a boundary eigenvalue problem. For the study of complete stellar models the singularity of the perturbation equations at the stellar center requires special attention. Therefore Gautschy \& Glatzel (1990b) have slightly modified the perturbation equations as given by Baker \& Kippenhahn 
(1962). We shall use them here in the form given by Gautschy \& Glatzel (1990b) (see also, Grott, 2003):

$$
\begin{gathered}
x^{2} \xi^{\prime}=A_{4}^{*}\left\{3 \xi+A_{5} p-A_{6} t\right\} \\
x^{2} l^{\prime}=A_{1}^{*}\left\{A_{10}^{*} \frac{d L_{0}}{d M} l-\left\{i \sigma+A_{10} A_{12}\right\} p+\left\{i \sigma A_{2}-A_{10} A_{11}\right\} t\right\} \\
p^{\prime}=-p-\xi\left\{4+A_{3} \sigma^{2}\right\} \\
t^{\prime}=\left\{A_{8} p-A_{9} t+A_{13} l-4 \xi\right\} A_{7}
\end{gathered}
$$

For better resolution, $\log P_{0}$ is used in Eqs. $2.12-2.15$ as the independent variable rather than the Lagrangian mass coordinate. The transformation from $m$ to $\log P_{0}$ is given by the equation for hydrostatic equilibrium of the background model. Accordingly, derivatives with respect to the independent variable $\left(\log P_{0}\right)$ are denoted by dashes (e.g., $\left.\xi^{\prime}, l^{\prime}\right)$. The dependent variables $\xi, l, p$ and $t$ correspond to the relative Lagrangian displacement and the relative perturbations of luminosity, pressure and temperature, respectively. $\sigma$ is the complex eigenfrequency normalized by the inverse of the global free fall time $\tau_{\mathrm{ff}}$ ( with $\tau_{\mathrm{ff}}=\sqrt{R^{3} / 3 G M}$ ) of the stellar model considered. For convenience the variables used are listed together with their physical meaning in Table 2.1.

The coefficients $A_{1 \ldots 13}$ appearing in Eqs. 2.12 - 2.15 depend on the background models and their stratification in the following way:

$$
\begin{gathered}
A_{1}=\frac{4 \pi r^{4} \delta P^{2}}{m \rho L}\left(\frac{4 \pi \bar{\rho}}{G}\right)^{\frac{1}{2}}, A_{2}=\frac{\rho T c_{p}}{P \delta}, A_{3}=\frac{4 \pi r^{3} \bar{\rho}}{m}, \\
A_{4}=\frac{r P}{G m \rho}, A_{5}=\alpha, A_{6}=\delta, \quad A_{7}=\nabla_{r a d}, A_{8}=\left(\frac{\partial \log \kappa}{\partial \log P}\right)_{T}, \\
A_{9}=4-\left(\frac{\partial \log \kappa}{\partial \log T}\right)_{P}, A_{10}=\frac{4 \pi r^{4} \epsilon P}{A_{1} G m L}, A_{10}^{*}=\frac{4 \pi r^{4} P}{A_{1} G m L}, \\
A_{11}=\left(\frac{\partial \log \epsilon}{\partial \log P}\right)_{T}, A_{12}=\left(\frac{\partial \log \epsilon}{\partial \log T}\right)_{P}, A_{13}=\frac{L}{L_{r a d}} .
\end{gathered}
$$

The linear perturbation equations (Eqs. 2.12 to 2.15) require four boundary conditions for their solutions. Two boundary conditions follow from the requirement that the solutions have to be regular in the integration interval between the center and the surface of the stellar model. In fact, the coefficients $\mathrm{A}_{1}$ and $\mathrm{A}_{4}$ become singular at the center and diverge as $\propto 1 / r^{2}$. To avoid singularities in the coefficients modified regular coefficients $A_{1}^{*}=x^{2} A_{1}$ and $A_{4}^{*}=x^{2} A_{4}$ are introduced and have been used in Eqs. 2.12 to 2.15. Here $x$ denotes the relative radius $(x=r / R)$. That the stellar center is a regular singular point of the differential system 2.12 to 2.15 is then deduced from the fact that the coefficient $x^{2}$ of the derivatives in Eqs. 2.12 and 2.13 vanishes for $x \rightarrow 0$. If the variables $\xi$ and $l$ are required to remain regular together with the left hand sides also 
the right hand sides of Eqs. 2.12 and 2.13 have to vanish at $x=0$ which is equivalent to the two algebraic relations:

$$
\begin{gathered}
3 \xi+A_{5} p-A_{6} t=0, \\
A_{10}^{*} \frac{d L_{0}}{d M} l-\left\{i \sigma+A_{10} A_{12}\right\} p+\left\{i \sigma A_{2}-A_{10} A_{11}\right\} t=0 .
\end{gathered}
$$

These relations obtained from the requirement of regularity are used as two boundary conditions to be satisfied by the solutions of the differential system at $x=0$ (see also, Gautschy \& Glatzel, 1990b; Grott, 2003).

The two remaining required boundary conditions are defined at the photosphere of the stellar model. As the photosphere is only the outer boundary of the stellar model but not the physical outer boundary of the star, these outer boundary conditions are ambiguous. The photosphere is characterized by Stefan-Boltzmann's law to hold there. Applying the process of linearisation to Stefan-Boltzmann's law we are left with the following algebraic relation

$$
4 t+2 \xi-l=0
$$

which can be used as a boundary condition for the perturbation equations at $x=1$. The second boundary condition at $x=1$ may be derived by requiring the Lagrangian density perturbation to vanish :

$$
\alpha p-\delta t=0
$$

Alternatively, the gradient of the relative pressure perturbation might be required to vanish (see Baker \& Kippenhahn, 1965):

$$
p+\xi\left\{4+A_{3} \sigma^{2}\right\}=0,
$$

As another alternative the outer boundary might be considered to be a force free boundary. Then the Lagrangian pressure perturbation has to vanish:

$$
p=0 .
$$

Due to the ambiguity of the outer boundary conditions, it is necessary to test the sensitivity of the results of the linear stability analysis to the outer boundary conditions. In fact, previous studies (see, e.g., Gautschy \& Glatzel, 1990b; Grott, 2003) have shown that the choice of the outer boundary conditions does not severely affect the final results of stability analyses. We shall discuss the dependence on boundary conditions of the investigations performed in this thesis later on.

\subsection{Solution of the linear pulsation equations}

\subsubsection{The adiabatic approximation}

Standard numerical schemes to solve the linear non adiabatic pulsation equations (2.12 to 2.15) have been described by Baker \& Kippenhahn $(1962,1965)$ and Castor $(1971)$. These schemes were sufficient to investigate and describe stability and pulsations of the classical pulsators as $\delta$ Cepheids and RR Lyrae stars which is mainly due to the fact 


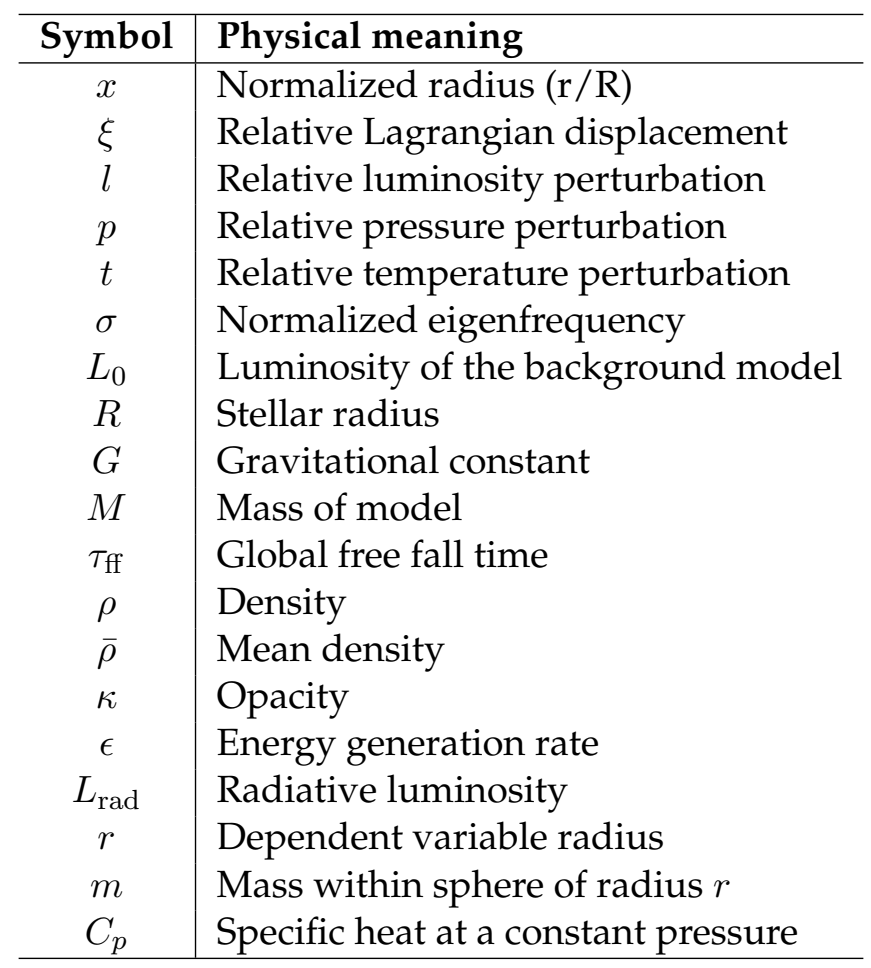

TABLE 2.1: List of variables and their physical meaning.

that for these stars the deviations from adiabatic behaviour are small. The standard techniques require an estimate for both the eigenfrequencies and the eigenfunctions which are usually taken from an adiabatic analysis. If the difference between nonadiabatic and adiabatic eigenfrequencies and eigenfunctions is small the standard approach will converge, for significant differences it fails (see also, Gautschy \& Glatzel, 1990a).

Physically, a mass element within a star is said to behave adiabatically, if it does not exchange heat within its surroundings during its motion. The motion is controlled by the dynamical timescale. Considering a mass shell of thickness $\Delta r$ within a star its local dynamical timescale $\left(\tau_{d y n}\right)$ is given by the sound travel time across the shell:

$$
\tau_{\mathrm{dyn}} \approx \frac{\Delta r}{c_{s}}
$$

where $c_{s}$ denotes the sound speed. On the other hand, the local thermal timescale $\left(\tau_{t h}\right)$ of the mass shell, i.e., the timescale on which the mass shell exchanges heat with its surroundings, is given by the ratio of its heat content and the local luminosity:

$$
\tau_{\mathrm{th}} \approx \frac{C_{p} T \Delta m}{L}
$$

where $\Delta m=4 \pi r^{2} \rho \Delta r$ is the mass of the shell. Both the local dynamical and the local thermal timescales are proportional to the thickness of the shell and therefore illdefined, whereas their ratio is a well defined quantity. For $\tau_{t h} / \tau_{d y n} \gg 1$ the mass shell will not significantly exchange heat with its surroundings during its motion and therefore behave adiabatically. Vice versa, for $\tau_{t h} / \tau_{d y n} \ll 1$ the heat exchange is faster than its dynamics implying large deviations from the adiabatic approximation.

Fig. 2.3 shows the ratio of the local thermal and dynamical timescales as a function 
of the relative radius for two stellar models representing a Cepheid and a HdC (Hydrogen deficient Carbon) star (see Gautschy \& Glatzel, 1990b). For any star close to its center this ratio attains very high values implying adiabatic behaviour there. Close to the surface, it is of order unity or even falls below unity for some stellar models (e.g., HdC stars). Thus significant deviations from adiabaticity are found for a Cepheid only in small range close to its surface. As a consequence, the adiabatic approximation provides good estimates for a nonadiabatic stability analysis in such cases. In contrast to Cepheids, for HdC stars the deviation from adiabaticity is significant and adiabatic guesses are not sufficient to guarantee the convergence of nonadiabatic stability analysis. In this case the standard techniques for nonadiabatic stability analyses fail and methods have to be applied which do not rely on adiabatic guesses. We shall introduce in the next subsection a method for nonadiabatic studies which does not need any guess for the eigenfrequency or the eigenfunction.

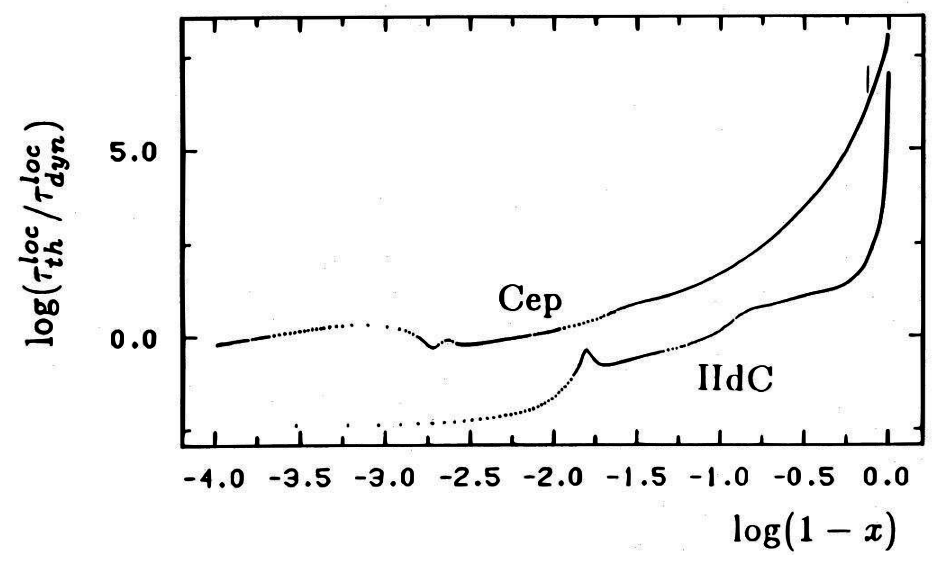

FIGURE 2.3: The ratio of local thermal and dynamical timescales as a function of the relative radius $(x)$ for two different stellar models corresponding to a Cepheid and a HdC star, adopted from Gautschy \& Glatzel (1990b).

\subsubsection{The Riccati method}

In this thesis, the linear perturbation equations (2.12 to 2.15) are solved using the Riccati method adapted to stellar stability problems by Gautschy \& Glatzel (1990a) and previously introduced by Scott (1973). In this approach the perturbation equations are treated as an initial value problem. However, such initial value problems for differential systems higher than second order are numerically unstable. To avoid this instability, the linear differential system is transformed into a stable nonlinear differential system with unique initial conditions. For the iteration of eigenfrequencies and eigenfunctions no external guesses are needed. The nonlinear differential system is obtained by defining vectors $\mathbf{u}$ and $\mathbf{v}$ according to:

$$
\mathbf{u}=\left[\begin{array}{l}
\xi \\
l
\end{array}\right] ; \quad \mathbf{v}=\left[\begin{array}{l}
p \\
t
\end{array}\right]
$$

The derivatives of these two vectors are then given by:

$$
\mathbf{u}^{\prime}=\left[\begin{array}{l}
\xi^{\prime} \\
l^{\prime}
\end{array}\right] ; \quad \mathbf{v}^{\prime}=\left[\begin{array}{l}
p^{\prime} \\
t^{\prime}
\end{array}\right]
$$


With these definitions the linear perturbation equations (2.12 to 2.15) can be expressed as:

$$
\begin{gathered}
\Lambda \mathbf{u}^{\prime}=\mathrm{C} \mathbf{u}+\mathrm{D} \mathbf{v} \\
\mathbf{v}^{\prime}=\mathrm{E} \mathbf{u}+\mathrm{F} \mathbf{v}
\end{gathered}
$$

where $\Lambda=\left[\begin{array}{cc}x^{2} & 0 \\ 0 & x^{2}\end{array}\right], C, D, E$ and $F$ are $2 \times 2$ matrices. The elements of the matrices $C$, $\mathrm{D}, \mathrm{E}$ and $\mathrm{F}$ can be read off from the perturbation equations (2.12 to 2.15). They depend on the eigenfrequency and the stratification of the background model. A $2 \times 2$ Riccati matrix $\mathcal{R}$ and its inverse $\mathcal{S}$ are introduced by:

$$
\begin{aligned}
& \mathbf{u}=\mathcal{R} \mathbf{v} \\
& \mathbf{v}=\mathcal{S} \mathbf{u}
\end{aligned}
$$

With these definitions, we obtain using equation 2.24 differential equations for the Riccati matrix and its inverse:

$$
\begin{gathered}
\Lambda \mathcal{R}^{\prime}=\mathrm{C} \mathcal{R}+\mathrm{D}-\Lambda \mathcal{R}(\mathrm{E} \mathcal{R}+\mathrm{F}) \\
\Lambda \mathcal{S}^{\prime}=\Lambda(\mathrm{E}+\mathrm{F} \mathcal{S})-\mathcal{S}(\mathrm{D} \mathcal{S}+\mathrm{C})
\end{gathered}
$$

Also the boundary conditions may be written in terms of matrices and vectors in the following way:

$$
\mathrm{J} \mathbf{u}=\mathrm{K} \mathbf{v}
$$

where $\mathrm{J}$ and $\mathrm{K}$ denote $2 \times 2$ matrices whose elements can be read off from the boundary conditions. Using equation 2.25 the matrix $\mathcal{R}$ and its inverse $\mathcal{S}$ can be expressed as:

$$
\begin{aligned}
\mathcal{R} & =\mathrm{J}^{-1} \mathrm{~K} \\
\mathcal{S} & =\mathrm{K}^{-1} \mathrm{~J}
\end{aligned}
$$

As $\mathrm{J}$ and $\mathrm{K}$ are completely determined by the boundary conditions, the Riccati matrices are also entirely determined at the boundaries. Thus unambiguous initial conditions for the integration of equations 2.26 or 2.27 as an initial value problem have been derived. Hence the boundary value problem has been transformed into a numerically stable initial value problem. The only free parameter in this approach is the complex eigenfrequency $\sigma$. Either equation 2.26 or 2.27 is integrated from both boundaries to some point $\mathrm{x}_{\text {fit }}$ within the integration interval thus providing two Riccati matrices $\left(\mathcal{R}^{\text {in }}\right.$ and $\mathcal{R}^{\text {out }}$ ) at $\mathrm{x}_{\text {fit }}$. The integration strategy is illustrated in Fig. 2.4. For an optimum resolution, the relative radius is used as independent variable for the inner integration, 
whereas $\ln P$ is used for the outer integration.

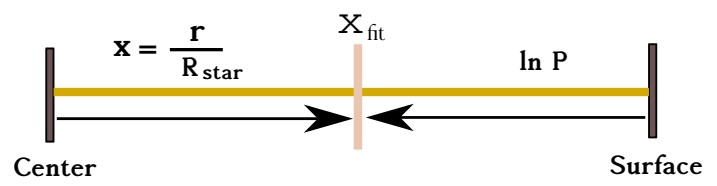

FIGURE 2.4: Sketch of the integration strategy for the integration of the Riccati equations (see also Fig. 1 in Gautschy \& Glatzel, 1990a).

At $\mathrm{x}_{\mathrm{fit}}$, the eigenfunction $\mathbf{u}$ and $\mathbf{v}$ have to be continuous which implies the following condition:

$$
\left[\mathcal{R}^{\text {in }}\left(\mathrm{x}_{\text {fit }}\right)-\mathcal{R}^{\text {out }}\left(\mathrm{x}_{\mathrm{fit}}\right)\right] \mathbf{v}=0
$$

In order to allow for a non-trivial solution, Eq. 2.31 has to satisfy the following condition:

$$
\operatorname{det}\left[\mathcal{R}^{\text {in }}\left(\mathrm{x}_{\mathrm{fit}}\right)-\mathcal{R}^{\text {out }}\left(\mathrm{x}_{\mathrm{fit}}\right)\right]=0
$$

Alternatively, a similar condition is derived for the matrix $\mathcal{S}$ :

$$
\operatorname{det}\left[\mathcal{S}^{\text {in }}\left(\mathrm{x}_{\mathrm{fit}}\right)-\mathcal{S}^{\text {out }}\left(\mathrm{x}_{\mathrm{fit}}\right)\right]=0
$$

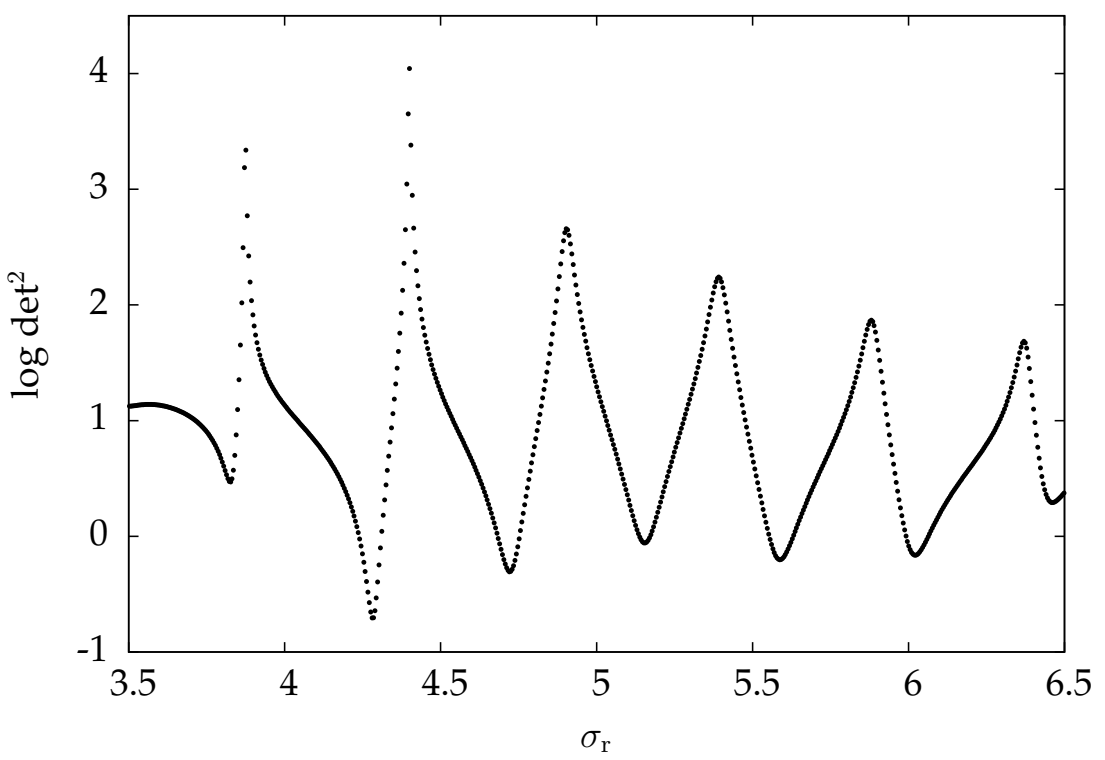

FIGURE 2.5: The Riccati determinant as a function of the real part $\left(\sigma_{\mathrm{r}}\right)$ of the eigenfrequency with fixed imaginary part $\sigma_{\mathrm{i}}=-0.5$ for a stellar model with parameter close to that of 55 Cygni. Local minima of the determinant function indicate the positions of the discrete eigenvalues.

They are used for initial guesses of the subsequent iteration.

The only free parameter contained in Eq. 2.32 or 2.33 is the complex eigenfrequency $\sigma$. Thus Eq. 2.32 or 2.33 provides a scalar complex equation, whose complex roots $\sigma$ are to be determined, and therefore may be regarded as the desired dispersion relation. 
Following Grott (2003), with this approach the determination of eigenfrequencies has been reduced to finding the roots of a complex equation. One of the major advantages of the Riccati method is that initial guesses for the eigenfrequencies can be obtained by examining the run of the determinant function Eq. 2.32 or 2.33 on the complex plane. Local minima of the determinant function can be used as initial guesses for subsequent iteration. We emphasize that initial guesses obtained in this way do not rely on any approximation of the perturbation problem (in particular not on the adiabatic approximation). Rather for these guesses already the entire set of equations is taken into account. For illustration, Fig. 2.5 shows the behaviour of the determinant function on a cut through the complex plane for a fixed imaginary part of the eigenvalue ( $\sigma_{\mathrm{i}}=$ -0.5 ) and a stellar model with parameters close to that of 55 Cygni (HD 198478). Local minima of the determinant function provide initial guesses for the subsequent iteration, where a complex secant method is used to iterate the eigenvalues (see also, Castor, 1971).

By considering a sequence of stellar models, the real parts $\sigma_{\mathrm{r}}$ of the eigenvalues determined (which correspond to the inverse of the pulsation period) and their imaginary parts $\sigma_{\mathrm{i}}$ (providing information about damping and excitation) may be presented as a function of stellar parameters, such as mass, effective temperature, luminosity and radius. Representations of this kind are usually referred to as "Modal Diagrams" (see, e.g., Saio et al., 1998). Fig. 2.6 shows a cartoon representation of a modal diagram containing five stellar models and a single mode. Modal diagrams contain information on the behaviour of the various modes as a function of stellar parameters. For example, mode interaction phenomena via avoided crossings and instability bands can be identified in modal diagrams. (In our normalization, unstable modes in a modal diagram can be identified by the negative imaginary part of their eigenfrequencies.) Further details will be discussed in connection with the results.
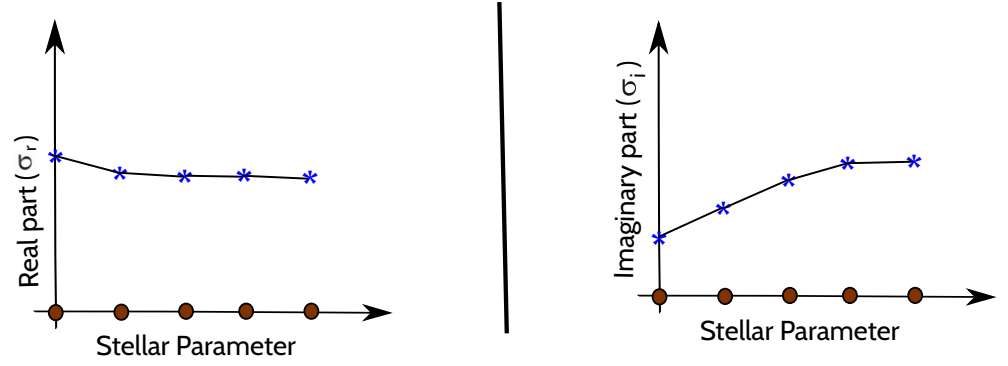

FIGURE 2.6: Cartoon representation of a modal diagram.

After having determined the eigenvalues and the Riccati matrix $\mathcal{R}$ as a function of the independent variable, Eq. 2.24 provides a differential equation for the calculation of the eigenfunction $\mathbf{v}$ :

$$
\mathbf{v}^{\prime}=\mathrm{E} \mathcal{R} \mathbf{v}+\mathrm{F} \mathbf{v}
$$

This equation for $\mathbf{v}$ is integrated from $\mathrm{x}_{\mathrm{fit}}$ to both the inner and the outer boundary, where the initial condition for $\mathbf{v}$ at $\mathrm{x}_{\mathrm{fit}}$ is given by Eq. 2.31. The remaining eigenfunction component $\mathbf{u}$ can then be derived using the definition of the Riccati matrix $\mathbf{u}=\mathcal{R} \mathbf{v}$. Eigenfunctions may be used to illustrate the relative variation of perturbations associated with the mode considered as a function of position within the stellar model given. 
As an example, real and imaginary parts of the relative Lagrangian displacement $(\xi)$ for a high order p-mode of a main sequence stellar model are shown in Fig. 2.7.

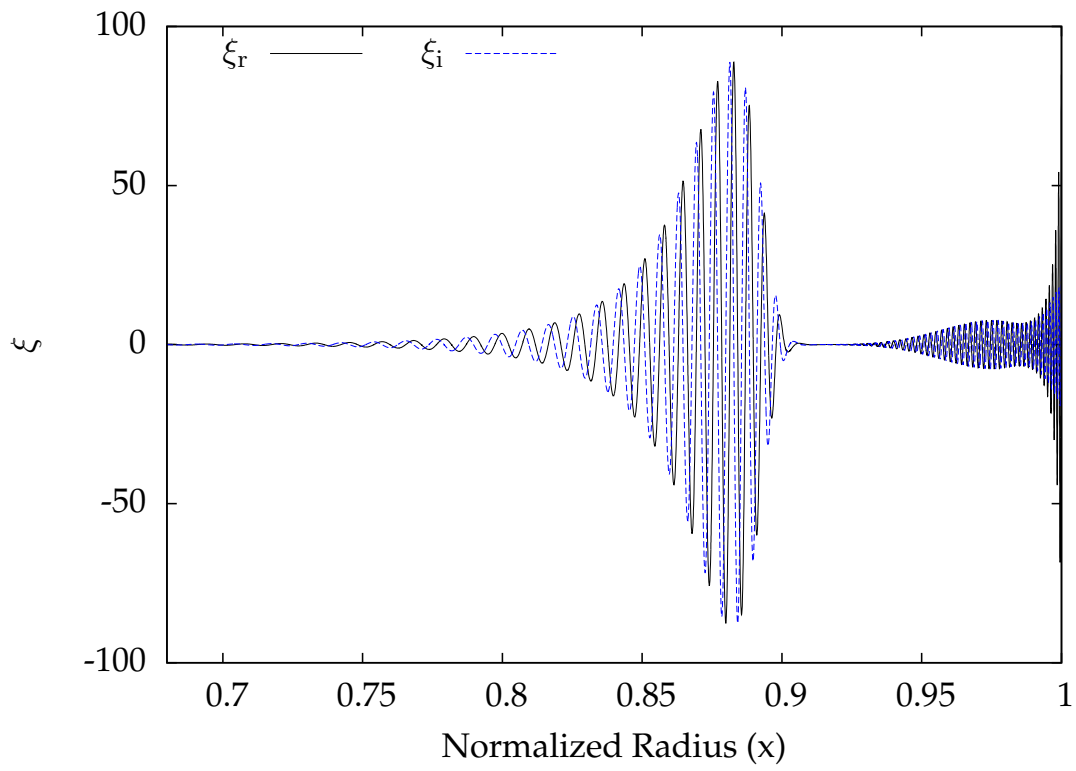

FIGURE 2.7: Real $\left(\xi_{\mathrm{r}}\right)$ and imaginary $\left(\xi_{\mathrm{i}}\right)$ parts of the Lagrangian displacement for a damped high order p-mode $\left(\sigma_{\mathrm{r}}=90.91\right.$ and $\left.\sigma_{\mathrm{i}}=2.74\right)$ of a massive main sequence stellar model as a function of relative radius.

Since the Riccati technique is a shooting method, it benefits from all the advantages of a shooting approach. In particular, the accuracy can be controlled locally to match any prescribed requirement without the necessity to increase the storage. Thus frequencies, growth and damping rates as well as eigenfunctions even of high order modes (see Fig. 2.7) can be reliably calculated with any desired precision.

\subsubsection{Strange modes}

Stellar instabilities are due to different physical processes. The classical $\kappa$ - and $\epsilon-$ mechanisms are based on a Carnot type heat engine (see, e.g., Aerts et al., 2010a; Cox, 1980). Hence thermodynamics is essential for modes excited by these mechanisms. For another group of unstable modes addressed as "strange modes" excitation by $\kappa$ - and $\epsilon$ - mechanism is entirely irrelevent. Typically, strange modes and associated instabilities have been found by stability analyses of stellar models having high luminosity to mass ratios (exceeding $10^{3}$ in solar unit). In modal diagrams strange modes exhibit a behaviour different from that expected for ordinary modes. Glatzel (1998) pointed out that the term strange mode is not precisely defined. According to the same author, "They are additional modes neither fitting in nor following the dependence on stellar parameters of the ordinary spectrum'. Modes of this kind were first described by Wood (1976) in a study of models for luminous helium stars. Due to their strange behaviour and unknown origin, Cox et al. (1980) addressed these modes as "strange" modes. Meanwhile, strange modes have been identified in various stellar models for, e.g., ZAMS objects (Glatzel \& Kiriakidis, 1993a; Kiriakidis et al., 1993), as well as RCrB, HdC (Saio \& Jeffery, 1988; Saio et al., 1984), AGB (Gautschy, 1993; Wood \& Olivier, 2014) and Wolf-Rayet stars (Glatzel \& Kaltschmidt, 2002; Glatzel et al., 1993; Kiriakidis et al., 1996). Apart from stellar models, strange mode instabilities are also present in 

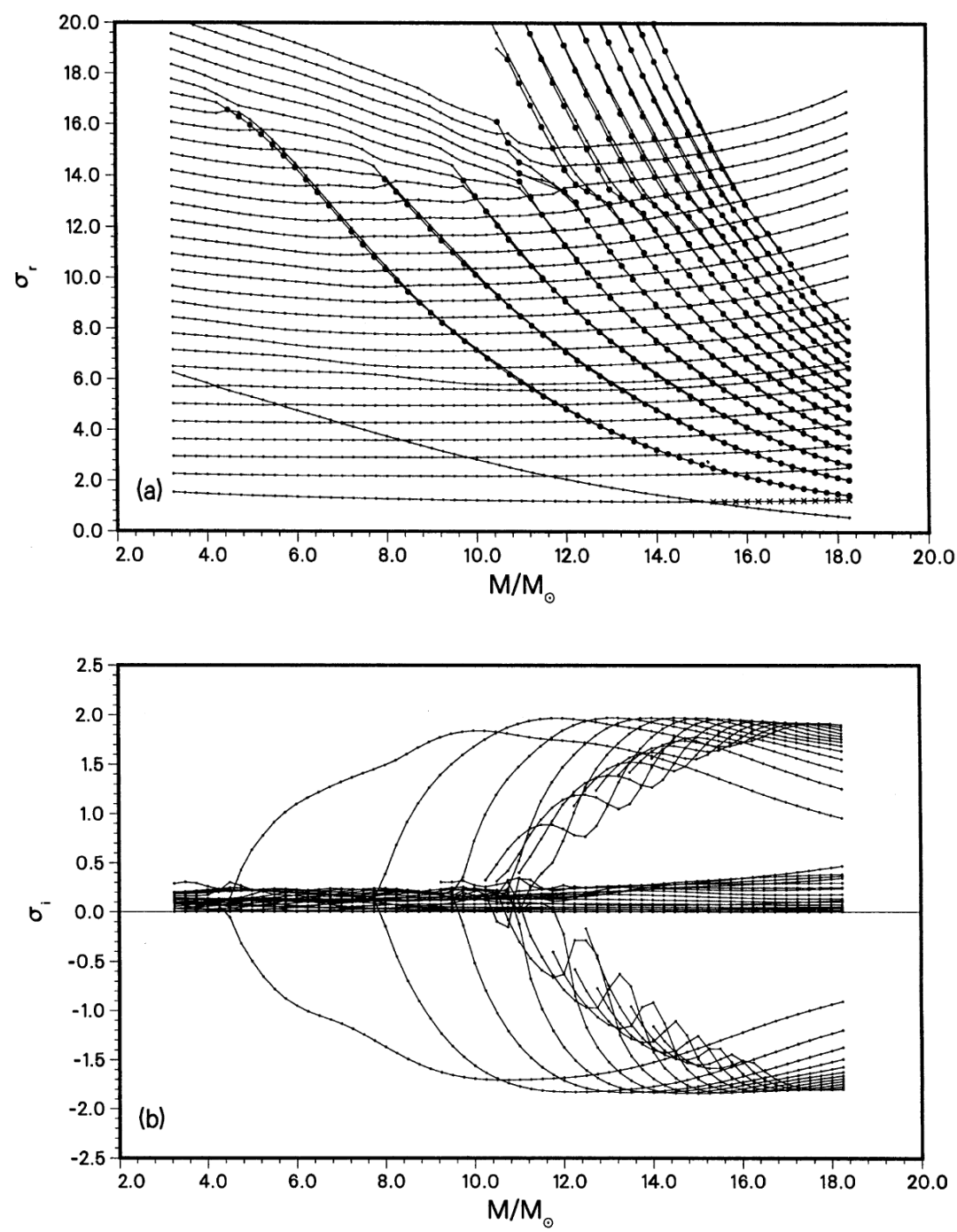

FIGURE 2.8: Modal diagram for models of Wolf-Rayet stars adopted from Glatzel et al. (1993). Real and imaginary parts of the eigenfrequencies normalized by the global free fall time are given as a function of mass. Thick dots in (a) and negative imaginary parts in (b) denote unstable modes. Note the appearance of strange modes and dynamical instabilities associated with them. 
models of accretion disks around stars and within galaxies (Glatzel \& Mehren, 1996). In spite of several attempts, the origin and properties of strange modes and associated instabilities are not yet fully understood.
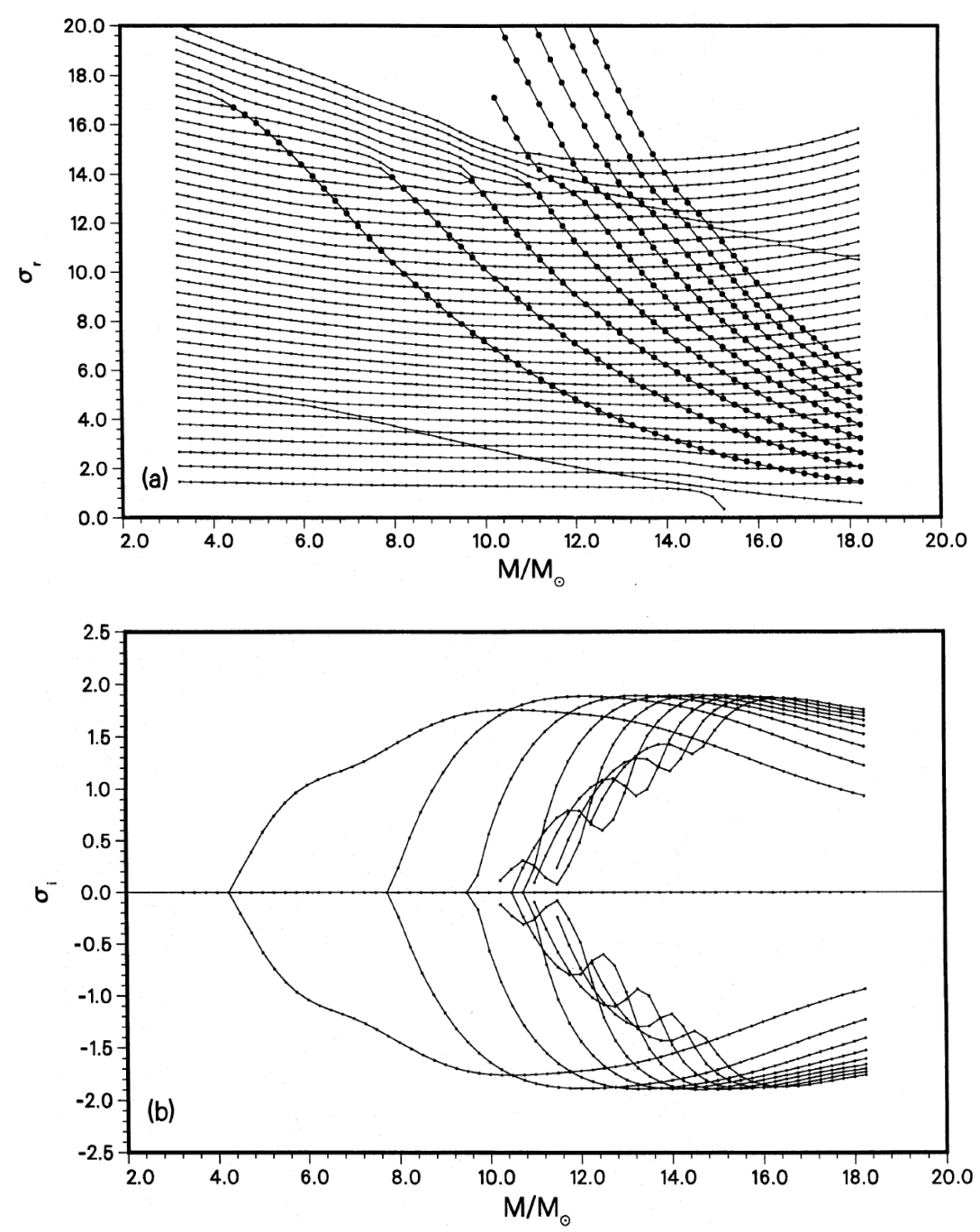

FIGURE 2.9: Same as Fig. 2.8 but within the NAR approximation adopted from Kiriakidis et al. (1996). Note the quality of the NAR approximation in particular with respect to the instabilities when comparing Figs. 2.8 and 2.9.

A prominent example for the occurrence of strange modes and associated instabilities are models for Wolf-Rayet stars (see Fig. 2.8, where the real and imaginary parts of the eigenfrequencies are given as a function of the mass of the stellar model). For these models the frequencies of ordinary damped modes only weakly depend on the stellar parameters. Contrary to ordinary modes, strange modes exhibit a sensitive dependence on stellar parameters (see Fig. 2.8, where the frequencies of strange modes decrease with mass). Moreover, the strange modes appear as almost complex conjugate pairs involving multiple dynamical instabilities.

Concerning the origin of the instabilities associated with strange modes, Glatzel (1994) claimed that the excitation is not due to the common $\kappa$ - or $\epsilon$ - mechanisms. A useful tool to identify the mechanism of instabilities is the Non Adiabatic Reversible 
(NAR) approximation introduced by Gautschy \& Glatzel (1990b). In the NAR approximation, the time derivative of the entropy perturbation is disregarded in the energy conservation equation. It implies that the heat capacity of the matter vanishes and heat can not be stored in the stellar envelope. As a consequence, luminosity perturbations vanish too. Thus any instability mechanism relying on a Carnot type heat engine cannot work within the NAR approximation. Should an instability still be present in the NAR approximation, it can neither be of thermal origin nor can it be based on a Carnot type process. For models of Wolf-Rayet stars, Kiriakidis et al. (1996) have shown that strange modes and associated instabilities are present both without approximations to the pulsation equations as well as in the NAR approximation. Fig. 2.9 is taken from Kiriakidis et al. (1996) and corresponds to the counterpart of Fig. 2.8 but within the NAR approximation. Even quantitatively the modal diagrams (Figs. 2.8 and 2.9) are very similar. Both ordinary and strange modes together with the associated instabilities appear in the same way both without and within the NAR approximation. In the NAR approximation, modes are either neutrally stable or come in complex conjugate pairs (Gautschy \& Glatzel, 1990b). Thus the damped ordinary modes in Fig. 2.8 become neutrally stable and the pairs of strange modes become exactly complex conjugate in the NAR approximation (Fig. 2.9). In the NAR approximation it is particularly obvious that strange modes form by mode pairing of ordinary modes thus providing a complex conjugate pair. This formation of strange modes from ordinary acoustic modes by the mode pairing process indicates that strange modes are of acoustic origin. The existence of strange modes in the NAR approximation proves them not to be of thermal origin. Moreover, the existence of strange mode instabilities in the NAR approximation proves them not to rely on a Carnot type process, in particular not on the classical $\kappa$ - or $\varepsilon$ - mechanisms. As a consequence, strange modes and instabilities, at least in Wolf-Rayet stars, have a mechanical origin.

Having identified mechanics as the origin of strange mode instabilities, their detailed mechanism still remains an open question. Under strictly adiabatic or isothermal conditions, the pressure perturbations $(\tilde{p})$ and the density perturbations $(\tilde{\rho})$ are proportional to each other, the coefficient of proportionality being the inverse of the square of the sound speed. As a consequence, there is no phase lag in a sound wave between pressure and density perturbations which indicate neutral stability. Considering the diffusion equation for energy transport, Glatzel (2001) shows that the density perturbation is proportional to the gradient of pressure perturbation:

$$
\tilde{\rho} \propto \frac{\partial \tilde{p}}{\partial r} .
$$

Thus, in a sound wave this kind of relation between pressure and density perturbation leads to a phase lag of $\pi / 2$ between the latter. This phase lag indicates either a damped or growing and thus unstable wave. The situation is intuitively similar to a pendulum with a phase lag between force and displacement. For more details, we refer to Glatzel (2001).

\subsection{Nonlinear Simulation}

Since in the present context, pulsation phenomenon is expressed as a homogeneous equations therefore exact pulsation amplitude can not be determined (see also, section 3.3.2.2 in Aerts et al., 2010a) 
A linear stability analysis only provides eigenfrequencies and associated eigenfunctions for the different modes of a stellar model. Final surface velocities and pulsation amplitudes cannot be determined by a linear theory. Due to nonlinear effects, pulsation periods may be affected in the nonlinear regime of the evolution of an instability and can therefore substantially differ from linearly determined periods. Therefore, in order to understand the final fate of an unstable model, following the instabilities into the nonlinear regime is inevitable. The method used to simulate the evolution of instabilities into the nonlinear regime is adopted from Grott et al. (2005).

\subsubsection{Basic assumption and equations}

According to Grott et al. (2005), the simulations of instabilities in the nonlinear regime implies the solution of the equations of mass conservation, momentum conservation, energy conservation and energy transport together with an equation of state. For this purpose, time $t$ and mass $m_{r}$ inside a radius $r$ are chosen as independent variables. For the simulation of stellar pulsations in the nonlinear regime we can restrict ourselves to the consideration of the envelope (see the discussion in section 2.1). For massive stars near the main sequence, energy transport in the envelopes is due to radiative diffusion and energy in the core is transported by convection (see e.g., Kippenhahn et al., 2012; Salaris \& Cassisi, 2006). However, envelopes of massive stars can also exhibit convection zones which are then associated with an opacity maximum. The interaction of convection and pulsation is poorly understood and still an open problem in astrophysics. Due to the lack of a reliable theory, in the present study the interaction of convection and pulsation is treated within the standard frozen-in approximation as introduced by Baker \& Kippenhahn (1965). It consists of neglecting the perturbation of the convective flux and is valid, if the contribution of convection to the total energy transport is negligible, and the timescale of pulsation is much shorter than the convective turn over timescale. In the envelopes of the models considered, the major fraction of the energy is transported by radiation diffusion and the the frozen-in approximation seems to be applicable. As an example, the ratio of the convective and the total luminosity is shown in Fig. 2.10 as a function of relative radius in the envelope of models for five representative massive main sequence stars. In Fig. 2.10 the contribution of the convective luminosity to the total luminosity never exceeds $10 \%$. For stellar models with a higher contribution of convective energy transport to the total luminosity a more sophisticated time dependent convection theory (TDC) is required (see the review by Houdek \& Dupret, 2015). Unfortunately, time dependent convection theories are still under development and the existing TDC formalisms suffer from many free parameters.

The equations describing the evolution of stellar instabilities into the nonlinear regime comprise the conditions of mass, momentum and energy conservation together with a prescription for the energy transport (see also, Glatzel et al., 1999; Grott et al., 2005):

$$
\begin{gathered}
\frac{d}{d t}\left(\frac{1}{\rho}\right)=\frac{\partial}{\partial m_{r}}\left(4 \pi r^{2} v\right) \\
\frac{d v}{d t}=-\frac{G m_{r}}{r^{2}}-4 \pi r^{2} \frac{\partial p}{\partial m_{r}}-v_{Q}
\end{gathered}
$$




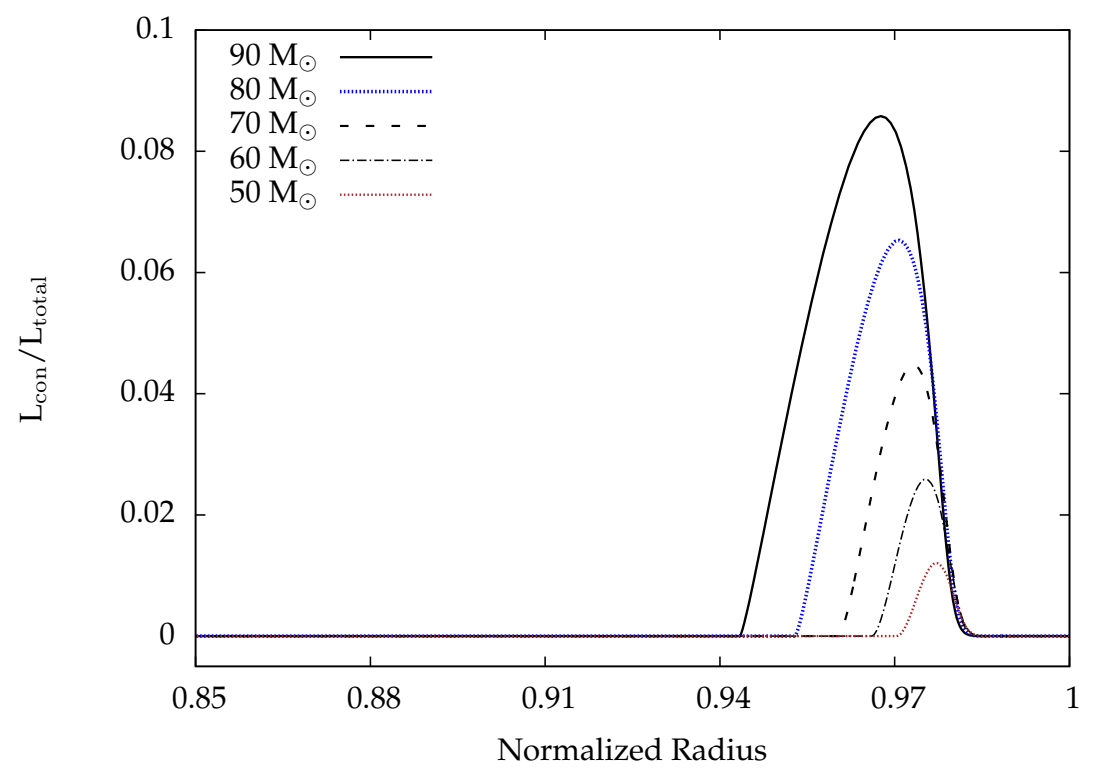

FIGURE 2.10: Ratio of convective and total luminosity $\left(\mathrm{L}_{\text {con }} / \mathrm{L}_{\text {total }}\right)$ as a function of relative radius in the envelopes of models for five massive main sequence stars with solar chemical composition.

$$
\begin{gathered}
\frac{d \epsilon}{d t}=-p \frac{\partial}{\partial m_{r}}\left(4 \pi r^{2} v\right)-\epsilon_{Q}-\frac{\partial}{\partial m_{r}}\left(4 \pi r^{2} F_{c o n}\right)-\frac{\partial}{\partial m_{r}}\left(4 \pi r^{2} F_{r a d}\right) \\
F_{r a d}=-4 \pi r^{2} \theta \frac{\partial p_{r a d}}{\partial m_{r}} .
\end{gathered}
$$

In this set of equations, time $t$ and mass $m_{r}$ (within radius $r$ ) are the independent variables. The symbols $\rho, r, v, p, p_{\text {rad }}, \epsilon$ and $G$ stand for density, radius, velocity, gas pressure, radiation pressure, specific internal energy and the gravitational constant, respectively. Viscous momentum transfer, viscous energy generation rate, radiative and convective flux are denoted by $v_{Q}, \epsilon_{Q}, F_{\text {rad }}$ and $F_{c o n}$, respectively. When acoustic instabilities are followed into the nonlinear regime, shock waves are expected to form. In order to handle shock waves in numerical calculations, an artificial viscosity is introduced in general. (see also, Noh, 1987; Von Neumann \& Richtmyer, 1950). The artificial viscous momentum transfer and the associated energy generation rate are introduced to smear out the discontinuities associated with shock waves. They should be present only in the vicinity of a shock and vanish elsewhere. In the equation for $F_{\text {rad }}, \theta=\frac{c}{\kappa}$ is the radiative diffusion coefficient expressed in terms of the speed of light $(c)$ and the opacity $\kappa$. Derivatives $\left(\frac{d}{d t}\right)$ denote Lagrangian time derivatives.

The system of equations $2.36-2.39$ has to be supplemented with prescriptions for the opacity and a thermal as well as a caloric equation of state. In this study both for the opacity and the equations of state tables have been used (see Iglesias \& Rogers, 1996; Rogers \& Iglesias, 1992; Rogers et al., 1996, for the OPAL opacity and the equation of state tables). Density $(\rho)$ and radiation pressure $\left(p_{\text {rad }}\right)$ are used as thermodynamic basis to get rid of the highly nonlinear dependence of the diffusion coefficient $(\theta)$ on temperature (see Grott et al., 2005). 


\subsubsection{Boundary conditions}

For the solution of the system of equations $2.36-2.39$ which represent a forth order system in the spatial variable $m_{r}$, four boundary conditions in space are required. As already discussed in the previous sections the pulsations considered do not affect the stellar core (see also Epstein, 1950). Therefore our considerations will be, similar to the linear stability analysis, restricted to the stellar envelopes. Two spatial boundary conditions are then imposed at the bottom of the envelope, two at its top, i.e., at the photosphere. Similar to the linear analysis, the photospheric boundary conditions are ambiguous as the photosphere of a model does not coincide with the physical boundary of a star. In order to allow shock waves to pass the outer boundary without being reflected, the following two boundary conditions have been found to be appropriate (Grott et al., 2005):

1. No heat storage at the outer boundary $\Rightarrow$

$$
\frac{\partial\left(r^{2} F\right)}{\partial m_{r}}=0
$$

2. The gradient of compression has to vanish at the outer boundary $\Rightarrow$

$$
\frac{\partial}{\partial m_{r}}\left(\frac{\partial\left(r^{2} v\right)}{\partial m_{r}}\right)=0
$$

Fig. 2.11 shows the propagation of shock waves at the outer boundary of a stellar model obtained by numerical simulations with these boundary conditions. Apparently no significant reflection of shocks at the outer boundary is found.
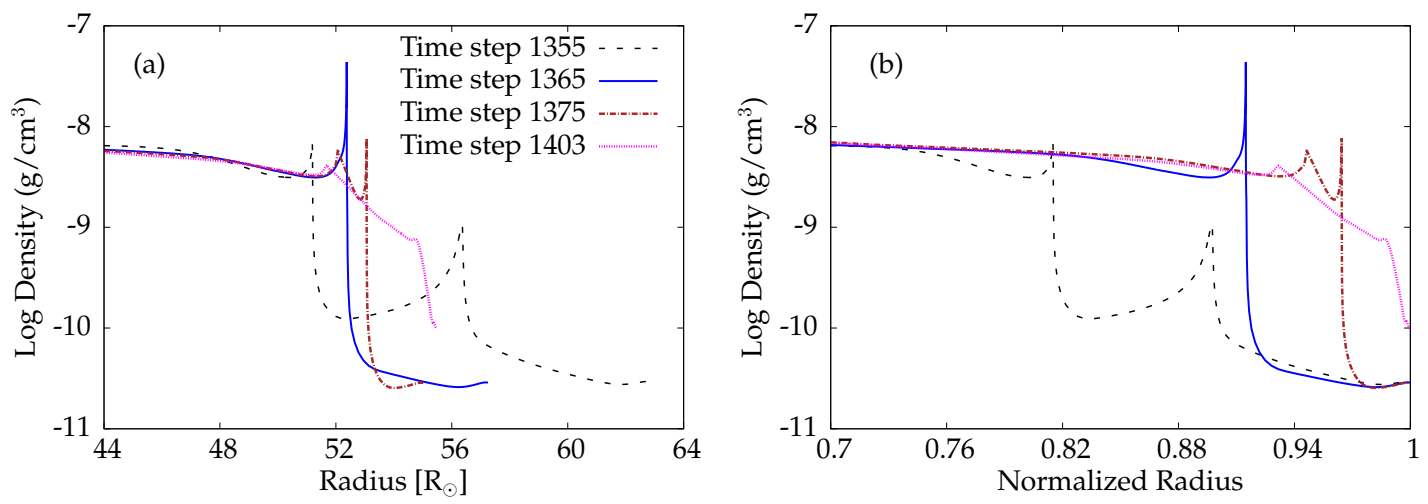

FIGURE 2.11: Propagation of shock waves near the outer boundary in a model for 55 Cygni with $19 \mathrm{M}_{\odot}$ and enhanced helium abundances. The density profile is shown as a function of radius in (a) and as a function of the radius normalized to its maximum contemporary value in (b) for various timesteps.

The bottom boundary of the envelope coincides with the outer boundary of the hydrostatic stellar core characterized by its constant mass and luminosity. Thus natural boundary conditions at the bottom of the envelope are a constant luminosity prescribed by the stellar core $\left(L=L_{\text {core }}\right)$ and vanishing velocity $(v=0)$. The radius of the inner boundary is determined by an (hydrostatic) envelope integration up to a maximum cutoff temperature of around $10^{7} \mathrm{~K}$. 


\subsubsection{Numerical scheme}

As analytical solutions for the system of Eqs. 2.36 - 2.39 are not available, a numerical scheme is needed for its solution. Concerning the energy balance of stellar pulsations, thermal and gravitational energies dominate and usually exceed the kinetic energy, which we are primarily interested in, by several orders of magnitude. Therefore the correct treatment of the energy balance is crucial for the dynamics of stellar pulsations. The problem is solved by adopting a numerical scheme which intrinsically satisfies the correct energy balance (see Grott et al., 2005). It turns out that such, with respect to the energy, fully conservative schemes necessarily have to be implicit in time. Even the artificial viscosity terms have to be written in a form which do not violate the energy balance.

Following Grott et al. (2005), the discretization of Eqs. 2.36 - 2.39 is done on a staggered grid, where vector valued quantities are defined on the nodes, while scalar, in particular thermodynamical, quantities are determined in the cells (for illustration see Fig. 2.12).

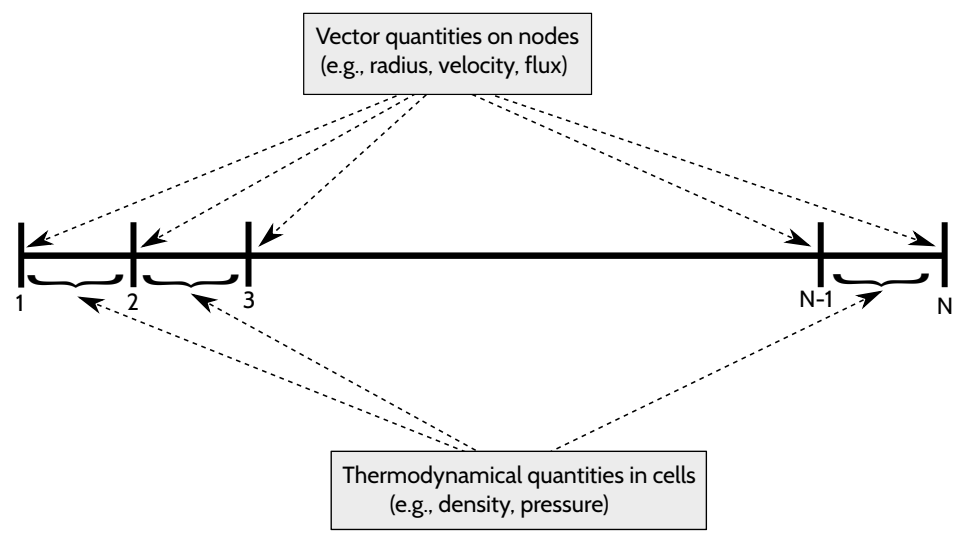

FIGURE 2.12: Definition of variables on the staggered grid with $\mathrm{N}$ nodes and N-1 cells. Vector valued quantities are defined on the nodes, scalar quantities in the cells.

The discretized form of Eqs. 2.36 - 2.39 reads in detail:

$$
\begin{gathered}
\left(\frac{1}{\hat{\rho}_{\mathrm{j}}}-\frac{1}{\rho_{\mathrm{j}}}\right) \frac{1}{\tau}=4 \pi \frac{R_{\mathrm{j}+1}^{2} v_{\mathrm{j}+1}^{(0.5)}-R_{\mathrm{j}}^{2} v_{\mathrm{j}}^{(0.5)}}{m_{\mathrm{j}}} \\
\frac{\hat{v}_{\mathrm{j}}-v_{\mathrm{j}}}{\tau}=-\frac{G m_{r, \mathrm{j}}}{\hat{r}_{\mathrm{j}} r_{\mathrm{j}}}-4 \pi R_{\mathrm{j}}^{2} \frac{p_{\mathrm{j}}^{(\alpha)}-p_{\mathrm{j}-1}^{(\alpha)}}{\bar{m}_{\mathrm{j}}}-v_{Q, \mathrm{j}} \\
\frac{\hat{\epsilon}_{\mathrm{j}}-\epsilon_{\mathrm{j}}}{\tau}=-4 \pi p_{\mathrm{j}}^{(\alpha)} \frac{R_{\mathrm{j}+1}^{2} v_{\mathrm{j}+1}^{(0.5)}-R_{\mathrm{j}}^{2} v_{\mathrm{j}}^{(0.5)}}{m_{\mathrm{j}}}-\epsilon_{Q, \mathrm{j}}-4 \pi \frac{R_{\mathrm{j}+1}^{2} F_{c o n, \mathrm{j}+1}-R_{\mathrm{j}}^{2} F_{c o n, \mathrm{j}}}{m_{\mathrm{j}}} \\
F_{r a d, \mathrm{j}}=-4 \pi r_{\mathrm{j}}^{2} \frac{p_{r a d, \mathrm{j}}-p_{r a d, \mathrm{j}-1} F_{r a d, \mathrm{j}+1}^{(\sigma)}-R_{\mathrm{j}}^{2} F_{r a d, \mathrm{j}}^{(\sigma)}}{m_{\mathrm{j}}} \sqrt{\theta_{\mathrm{j}} \theta_{\mathrm{j}-1}}
\end{gathered}
$$


In the difference equations 2.42 to $2.45, \tau$ denotes the variable integration time step. Quantities with a hat (e.g., $\hat{\rho}, \hat{v}, \hat{\epsilon})$ correspond to the next time step, quantities without hat (e.g., $\rho, v, \epsilon)$ are evaluated at the current time step. For a quantity $c_{\mathrm{j}}$ a time average with weight $\alpha$ is defined as $c_{\mathrm{j}}^{(\alpha)}=\alpha \hat{c}_{\mathrm{j}}+(1-\alpha) c_{\mathrm{j}}$. For the weights $\alpha$ and $\sigma, \alpha=\sigma=1$ has been adopted in the present study. Subscripts $\mathrm{j}$ denote the number of the node or the cell, respectively. $m_{r, \mathrm{j}}$ is the mass inside the radius $r_{\mathrm{j}}$ including the mass of the core. $m_{\mathrm{j}}$ denotes the mass withing cell $\mathrm{j}, \bar{m}_{\mathrm{j}}=1 / 2\left(m_{j+1}+m_{j}\right)$ may be regarded as the mass defined on node $\mathrm{j}$. The quantity $R_{\mathrm{j}}$ is defined to satisfy the conservativity of the scheme in the following way:

$$
R_{\mathrm{j}}^{2}=\frac{\hat{r}_{\mathrm{j}}^{2}+\hat{r}_{\mathrm{j}} r_{\mathrm{j}}+r_{\mathrm{j}}^{2}}{3} .
$$

The system of difference equations $2.42-2.45$ is closed by the equations of state $p_{\mathrm{j}}=$ $p\left(p_{r a d, \mathrm{j}} ; \rho_{\mathrm{j}}\right)$ and $\epsilon_{\mathrm{j}}=\epsilon\left(p_{\text {rad }, \mathrm{j}} ; \rho_{\mathrm{j}}\right)$ together with a prescription for the opacity $\kappa_{\mathrm{j}}=$ $\kappa\left(p_{\text {rad }, \mathrm{j}} ; \rho_{\mathrm{j}}\right)$. For more details we refer to Grott et al. (2005).

\subsubsection{Validation of the scheme}

The validation of the numerical scheme adopted is crucial concerning the reliability of the results. One problem concerns the resolution provided by the Lagrangian grid,

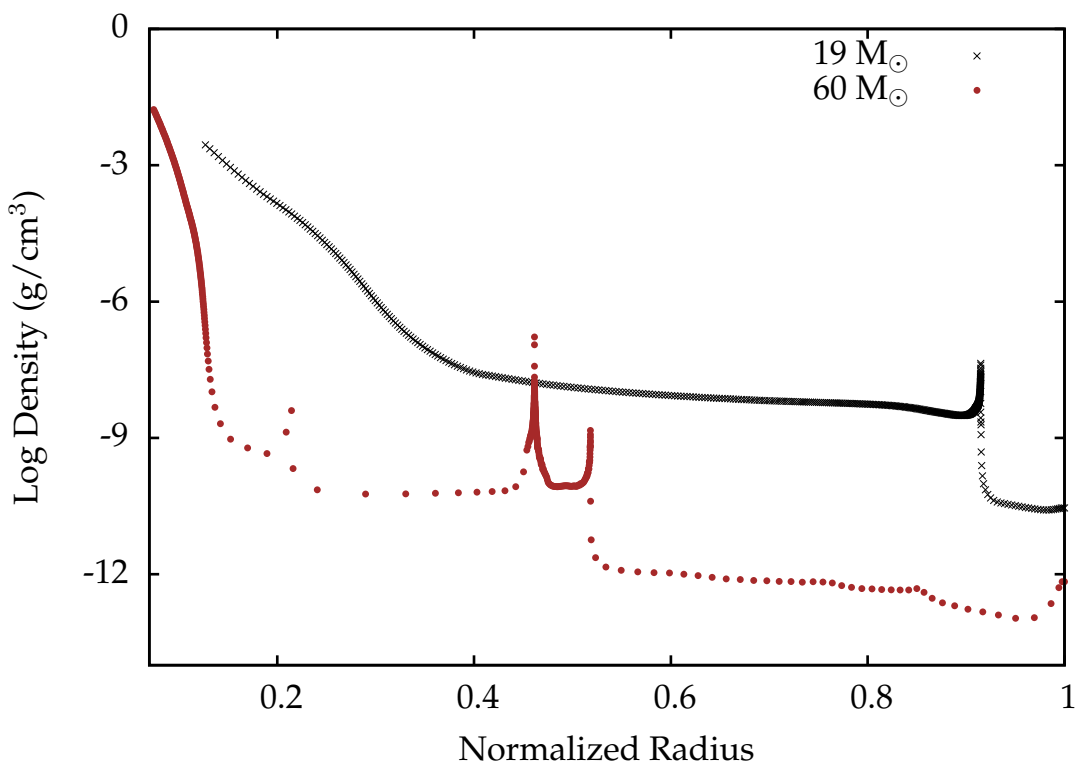

FIGURE 2.13: Snapshots of the density stratification as a function of normalized radius (normalization by the contemporary maximum radius) for two stellar models during finite amplitude pulsations. Note the different distribution of the 500 grid points used.

in particular, if shock waves are present. For illustration, the distribution of the 500 grid points used is shown in Fig. 2.13 for an instance of time during finite amplitude pulsations of two stellar models. For the less massive model, the structure of the star including the shock wave is well resolved. However, for the massive model, two of the three shock waves have attracted almost all grid points, thus being well resolved themselves but leaving only a few grid points for the third shock and the remaining part of 
the envelope. Thus a large part of the model is not sufficiently well resolved, which is a consequence of the Lagrangian grid moving together with the mass elements. To solve the problem, grid points have to be inserted in underresolved regions, i.e., the Lagrangian grid has to be reconstructed. Attempts towards a conservative grid reconstruction procedure have been done by Chernigovski et al. (2004). However, a fully satisfactory grid reconstruction approach is still not available. In the present study we shall restrict ourselves to sufficiently well resolved models obtained with a constant number of grid points.

Another problem concerns the artificial viscosity introduced to smooth the discontinuities appearing during the propagation of shocks. To determine the right amount of artificial viscosity is important for the quality of the results. For example, if the artificial viscosity is too high, it can damp the physical instabilities. On the other hand, if it is too small, numerical oscillations around the shock will occur (Gibb's phenomenon), and finally the shock waves can no longer be handled. Therefore, in order to avoid numerical artifacts, the artificial viscosity has to be chosen with care.

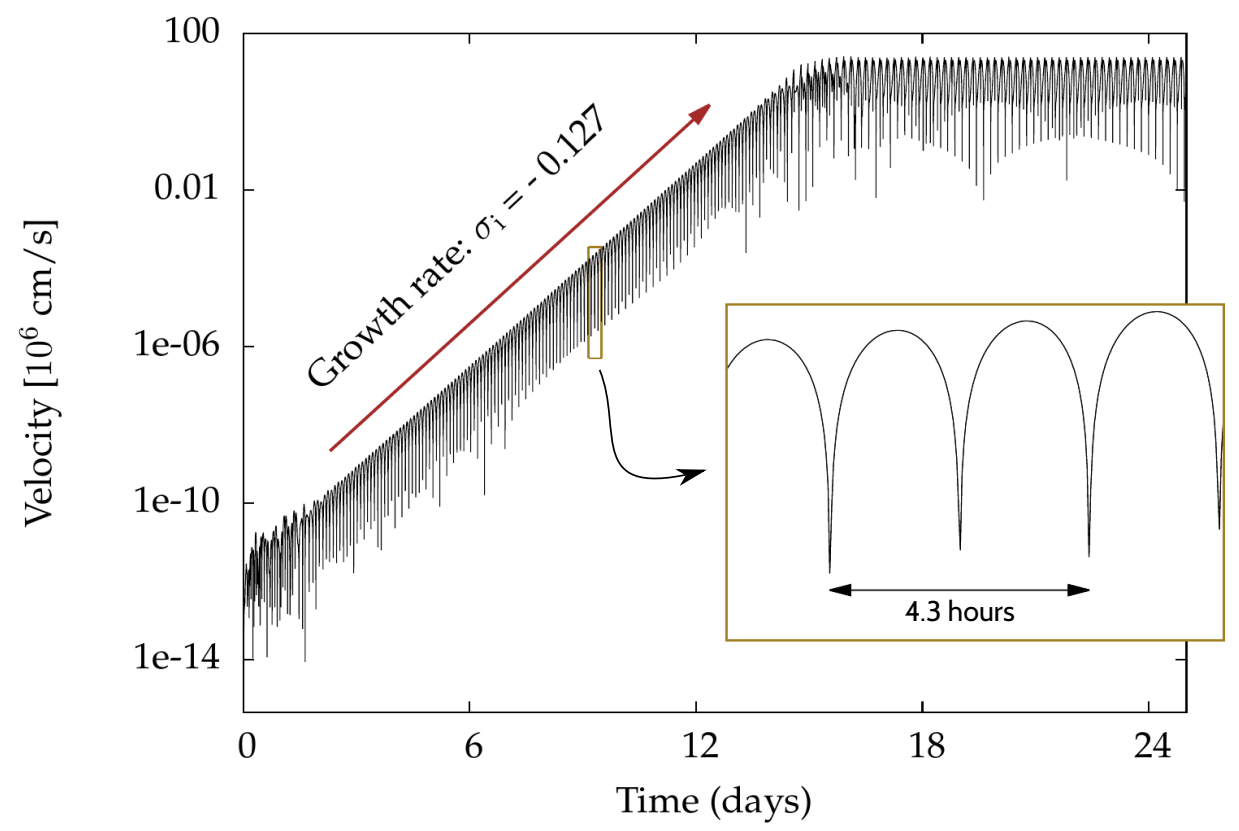

FIGURE 2.14: Simulation of the evolution of an instability for a $90 \mathrm{M}_{\odot}$ ZAMS model from hydrostatic equilibrium into the nonlinear regime. The photospheric velocity is given as a function of time. The evolution is initiated by numerical noise, undergoes the linear phase of exponential growth, and saturates in the nonlinear regime. Within the linear phase the pulsation period and the growth rate can be compared with the results of an independent linear stability analysis for validation.

A crucial test for the nonlinear simulation code is its behaviour in the initial phase of the evolution of a physical instability of an unstable model. Starting from an unstable model in hydrostatic equilibrium as an initial condition the code should pick up one or more unstable modes with correct periods and growth rates (as predetermined by an independent linear stability analysis) from numerical noise without any further external perturbation. In particular, it should exhibit the linear phase of exponential growth of the instabilities, where the periods and growth rates predicted by the linear theory can be compared with the results of the simulations which is used to validate 

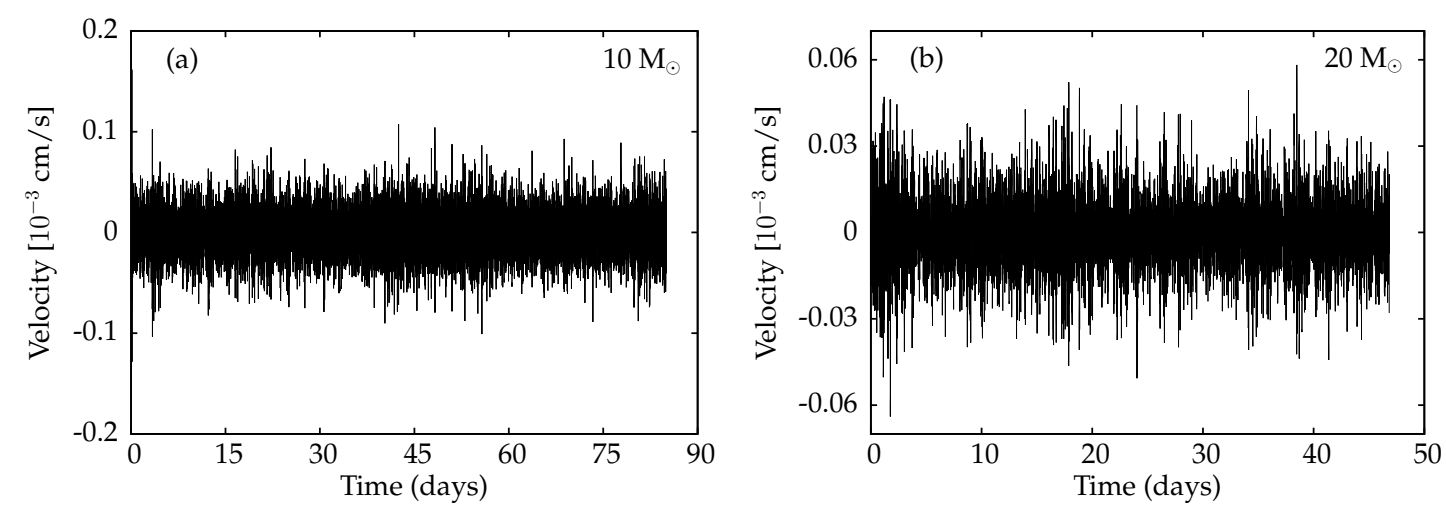

FIGURE 2.15: Photospheric velocity as a function of time for two stable ZAMS models with a mass of $10 \mathrm{M}_{\odot}$ (a) and $20 \mathrm{M}_{\odot}$ (b), respectively. The models remain in hydrostatic equilibrium and the velocities shown correspond to numerical noise.

the code. The succesfull test proves that the simulated evolution is governed by physical instabilities and not due any numerical instabilities or artifacts. As an example, Fig. 2.14 shows the photospheric velocity as a function of time of an unstable ZAMS model with a mass of $90 \mathrm{M}_{\odot}$ as obtained by a simulation. The simulation starts in hydrostatic equilibrium with a velocity amplitude of the order of $10^{-5} \mathrm{~cm} / \mathrm{s}$. After the linear phase of exponential growth it saturates in the nonlinear regime with an amplitude of approximately $50 \mathrm{~km} / \mathrm{s}$ after 16 days. An independent linear stability analysis provides the real $\left(\sigma_{\mathrm{r}}\right)$ and imaginary $\left(\sigma_{\mathrm{i}}\right)$ parts of the eigenfrequencies associated with the most unstable mode as:

- $\sigma_{\mathrm{r}}=2.193$ (corresponding to a period of 4.38 hours)

- $\sigma_{\mathrm{i}}=-0.129$

The growth rate determined from the simulation (see, Fig. 2.14) corresponds to $\sigma_{\mathrm{i}}=-$ 0.127 and the period in the linear phase of exponential growth is found to be 4.3 hours ( corresponding to $\sigma_{\mathrm{r}}=2.2$ ). Both the period and the growth rate as obtained from the simulation match the period and the growth rate determined by the linear stability analysis. Hence the final result of the simulations in the nonlinear regime have to be regarded as the consequence of the physical instabilities of the model.

Simulations of unstable models have been demonstrated to pick the correct period and growth rate corresponding to the most unstable mode from numerical noise without any external perturbation. Vice versa, the simulation scheme might be tested by considering a stable hydrostatic model. In this case the model should remain in hydrostatic equilibrium and the velocity amplitudes should stay on the numerical noise level. As an example, Fig. 2.15 shows the velocity as a function of time as obtained by simulation for two stable hydrostatic ZAMS models with solar chemical composition. As expected for a successful test, the photospheric velocity never exceeds the noise level of $10^{-4} \mathrm{~cm} / \mathrm{s}$ (see, Fig. 2.15). Thus the scheme is proven to be sensitive to physical instabilities and simultaneously does not suffer from numerical instabilities and artifacts. 


\subsection{Nonradial perturbations}

So far we have restricted ourselves to considering radial perturbations. Nonradial pulsations have been identified in a variety of stars. In this context, radial pulsations are considered as a special case of nonradial pulsations with zero harmonic degree (see also, Chapter 1 of Unno et al., 1979). These authors point out that the 'richness of nonradial oscillations ${ }^{1}$ ' is due to the pressure as well as the gravity as a restoring force. In the case of radial pulsations only the pressure can act as a restoring force. On the basis of the restoring forces, nonradial pulsations fall into two groups:

- Pressure modes (p-modes): pressure acts as restoring force

- Gravity modes (g-modes): buoyancy acts as restoring force

In general nonradial modes have a mixed character. However, in certain situations one can distinguish between pressure and gravity modes on the basis of two characteristic frequencies, the Lamb frequency $\left(L_{l}\right)$ and the Brunt-Väisälä frequency $(\mathrm{N})$. If the frequency of a mode is larger than both of the characteristic frequencies, it is a p-mode. In the opposite case (the frequency is smaller than both of the characteristic frequencies), the mode is identified as a g-mode.

In this study, we have adopted the equations governing linear nonadiabatic nonradial pulsations in the form provided by Glatzel \& Gautschy (1992). This set of equations consists of the linearized forms of mass, momentum and energy conservation, Poisson's equation for the gravitational potential, the energy transport equation and an equation of state. The angular dependence of the dependent variables is separated by an expansion in terms of spherical harmonics. Introducing the eigenfrequency similar to the radial case, the perturbation equations for nonadiabatic nonradial pulsations are reduced to a sixth order ordinary differential boundary eigenvalue problem with the harmonic degree $l$ of the perturbation as a parameter. The order of the differential system might be reduced by Cowling's approximation (see also, Cowling, 1941). It consists of neglecting the perturbations of the gravitational potential which reduces the sixth order problem to a forth order boundary eigenvalue problem. In our study, the complete set of the nonadiabatic nonradial sixth order pulsation equations are taken into account. Boundary conditions are applied at the photosphere and at the bottom of the stellar envelope. The sixth order boundary eigenvalue problem is solved similar to the radial case by using the Riccati method (see also, Gautschy \& Glatzel, 1990a).

\footnotetext{
${ }^{1}$ Here oscillations and pulsations are synonyms
} 



\section{Chapter 3}

\section{Massive main sequence stars ${ }^{1}$}

The chemical evolution of galaxies and their energy balance is governed to a large extent by massive stars (Matteucci, 2008; Nomoto et al., 2013). Moreover, they are most probably responsible for the re-ionization of the universe (see e.g., Barkana \& Loeb, 2001; Ciardi \& Ferrara, 2005; Haehnelt et al., 2001). In this respect the question, whether an upper limit for the mass of a star exists, is of fundamental importance. Two issues which can imply an upper limit have been discussed so far. Either the star formation process might not allow stars to become more massive than a critical mass or a stellar instability for stars more massive than a certain limit excludes their existence. In the present study we consider the second possibility.

The investigation of the stability of massive stars with respect to infinitesimal radial perturbations dates back to Ledoux (1941). As the cause of instability, he considered the $\varepsilon$ - mechanism associated with nuclear energy production in the stellar core and derived a critical mass of $100 \mathrm{M}_{\odot}$, above which main sequence stars are unstable with respect to this process. Later on, a refinement of this study by Schwarzschild \& Härm (1959) has provided a new stability limit of $60 \mathrm{M}_{\odot}$. That the stability limit for massive stars caused by the $\varepsilon$ - mechanism implies severe consequences for the existence and evolution of massive stars has been questioned by Ziebarth (1970). He argued that the range of $\varepsilon$ - unstable stellar models is restricted to the very vicinity of the zero age main sequence (ZAMS) and that the growth timescale of the $\varepsilon$ - instability competes with the stellar evolution timescale. Thus the star has left the instability domain by evolution before the instability has grown substantially. More recently, these arguments were confirmed by Baraffe et al. (2001) and Sonoi \& Umeda (2012) on the basis of an investigation of primordial stars providing a stability limit of $120 \mathrm{M}_{\odot}$ for these objects. Moreover, $\varepsilon$ - instability is connected with the radial fundamental mode. According to the study by Shiode et al. (2012) convective damping can possibly overcome excitation by the $\varepsilon$ - mechanism of this mode. As a consequence, we conclude that $\varepsilon$ - instability does not pose a severe problem for the existence and evolution of massive stars.

Using OPAL opacity (Iglesias \& Rogers, 1996; Rogers \& Iglesias, 1992; Rogers et al., 1996) tables, Glatzel \& Kiriakidis (1993a) have performed a linear stability analysis of ZAMS models with masses between 40 and $120 \mathrm{M}_{\odot}$. These authors identified instabilities with growth rates in the dynamical range which are associated with the occurrence of strange modes (for the definition of strange modes see, Gautschy, 1992; Gautschy \& Glatzel, 1990b; Glatzel, 2001; Saio \& Jeffery, 1988; Wood, 1976). For these modes metallicity dependent stability limits between 80 and $58 \mathrm{M}_{\odot}$ have been derived.

In connection with the $\varepsilon$ - instability, nonlinear simulations have been performed with contradictory results to determine the final fate of the unstable models (Appenzeller, 1970; Papaloizou, 1973a,b; Talbot, 1971a,b; Ziebarth, 1970). For example, Appenzeller (1970) claims the instability to lead to a pulsationally driven wind with a mass

\footnotetext{
${ }^{1}$ A modified version of this chapter has been published in MNRAS (Yadav \& Glatzel, 2017).
} 
loss rate of up to $4 \times 10^{-5} \mathrm{M}_{\odot} / \mathrm{yr}$, whereas Papaloizou (1973a,b) does not find any mass loss and estimates an upper limit for the mass loss rate of $10^{-6} \mathrm{M}_{\odot} / \mathrm{yr}$. The latter would support the arguments discussed above against $\varepsilon$ - instability as an essential effect for the structure and evolution of massive stars.

With respect to the strange mode instabilities of ZAMS models, up to now no nonlinear simulations have been performed to identify their final result. In the present study, we intend to reinvestigate the linear stability of ZAMS models with solar chemical composition and masses in the range between 50 and $150 \mathrm{M}_{\odot}$. On the basis of this analysis we shall select models for nonlinear simulations and follow the strange mode instabilities into the nonlinear regime in order to determine their final consequences. Whether the instabilities lead to a pulsationally driven wind, will be of particular interest. An estimate of the associated mass loss rate will then provide information on the implications of the instabilities on stellar structure and evolution.

In section 3.1 the stellar models considered will be discussed. Their linear stability analysis and its results are described in sections 3.2 and 3.3, respectively. In section 3.4 we comment on the nonlinear simulations in general, section 3.5 contains their results for selected stellar models. A discussion and our conclusions follow.

\subsection{Stellar models}

We restrict our studies to the upper zero age main sequence (ZAMS) with masses in the range between 50 and $150 \mathrm{M}_{\odot}$, where rotation and magnetic fields are disregarded. Solar chemical composition $(X=0.70, Y=0.28, Z=0.02)$ has been adopted for all models. The MESA code (Paxton et al., 2011, 2013, 2015) has been used to determine effective temperatures and luminosities as a function of mass along the ZAMS, where the ZAMS is defined as the stage when one percent of the hydrogen has been burnt in the center. For selected values of the mass, effective temperatures and luminosities are listed in table 3.1.

For the subsequent stability analysis we consider stellar envelope models: As long as $\varepsilon$ - instability is not of interest, the stellar core does not participate in stellar pulsations and can, together with the nuclear processes, be disregarded in the investigation. The bottom of the envelope is defined by a suitably chosen cut off temperature of the order of $10^{7} \mathrm{~K}$. On the basis of the ZAMS parameters mass, effective temperature and luminosity, envelopes have been constructed by initial value integration from the photosphere to the cut off temperature. As additional initial conditions StefanBoltzmann's law was imposed and the photospheric pressure was prescribed at the photosphere. For the opacity, the OPAL tables (Iglesias \& Rogers, 1996; Rogers \& Iglesias, 1992; Rogers et al., 1996) have been used and Schwarzschild's criterion has been adopted to determine the onset of convection. Convection is treated according to standard mixing length theory (Böhm-Vitense, 1958) with 1.5 pressure scale heights for the mixing length.

\subsection{Linear stability analysis}

For the linear stability analysis with respect to radial perturbations of the ZAMS models described, we have adopted the perturbation equations in the form given by Gautschy \& Glatzel (1990b). The Riccati method has been used to solve these equations (see also, Gautschy \& Glatzel, 1990a). Since a theory for the interaction of pulsation and convection does not yet exist, we have used the "frozen in approximation" as introduced by 
TABLE 3.1: Mass, effective temperature and luminosity on the zero age main sequence for solar chemical composition.

\begin{tabular}{lcr}
\hline $\mathrm{M}\left(M_{\odot}\right)$ & $\log \left(\mathrm{T}_{e f f}\right)$ & $\log \left(\frac{L}{L_{\odot}}\right)$ \\
\hline 50 & 4.656 & 5.571 \\
60 & 4.670 & 5.726 \\
70 & 4.679 & 5.850 \\
80 & 4.685 & 5.954 \\
90 & 4.688 & 6.042 \\
100 & 4.688 & 6.120 \\
150 & 4.672 & 6.398 \\
\hline
\end{tabular}

Baker \& Kippenhahn (1965) for the treatment of convection (see also, Li, 2000; Saio \& Wheeler, 1985). In this approximation, the Lagrangian perturbation of the convective flux is disregarded. It holds, if the pulsation timescale is much shorter than the convective turn over timescale, and if the contribution of the convective flux to the total flux is small. These conditions are met in the models considered. We note that according to Sonoi \& Shibahashi (2014) strange mode instabilities are still present in models of massive stars, if a time dependent treatment of convection is adopted.

The perturbation equations together with appropriate boundary conditions pose a boundary eigenvalue problem with complex eigenvalues and eigenfunctions. The complex eigenfrequencies $\left(\sigma=\sigma_{r}+i \sigma_{i}\right)$ discussed below are normalized with the global free fall time $\tau_{\mathrm{ff}}$ defined by

$$
\tau_{\mathrm{ff}}=\sqrt{\frac{\mathrm{R}^{3}}{3 \mathrm{GM}}},
$$

where $\mathrm{R}$ denotes the stellar radius, $\mathrm{G}$ stands for the gravitational constant, and $\mathrm{M}$ is the mass of the star. The real part of the eigenfrequency corresponds to the pulsation frequency, while the imaginary part provides information on the damping or excitation of a mode. In the normalization adopted, negative values of the imaginary part correspond to unstable modes, positive values indicate damping.

\subsection{Results of the linear stability analysis}

The results of a linear stability analysis of the stellar envelope models described are conveniently presented in the form of "modal diagrams" (see, e.g., Saio et al., 1998), where the eigenfrequencies are given as a function of stellar parameters, such as mass and effective temperature. In Fig. 3.1 real and imaginary parts of the eigenfrequencies are shown as a function of mass along the ZAMS. This modal diagram contains both ordinary and strange modes. Ordinary modes are characterized by a weak dependence on stellar parameters of the real part of the eigenfrequencies, whereas strange modes exhibit a stronger variation. Thus multiple mode crossings are generated which unfold into avoided crossings or instability bands. A weak instability is associated with the ordinary fundamental mode for masses above $116 \mathrm{M}_{\odot}$, whereas a dynamical strange mode instability sets in above $58 \mathrm{M}_{\odot}$, whose growth rate increases with mass. Only low order modes are affected by instability. Both concerning the mass range of unstable models and the growth rate, the instability of the ordinary fundamental mode is entirely negligible. Thus stability studies considering the fundamental mode only 

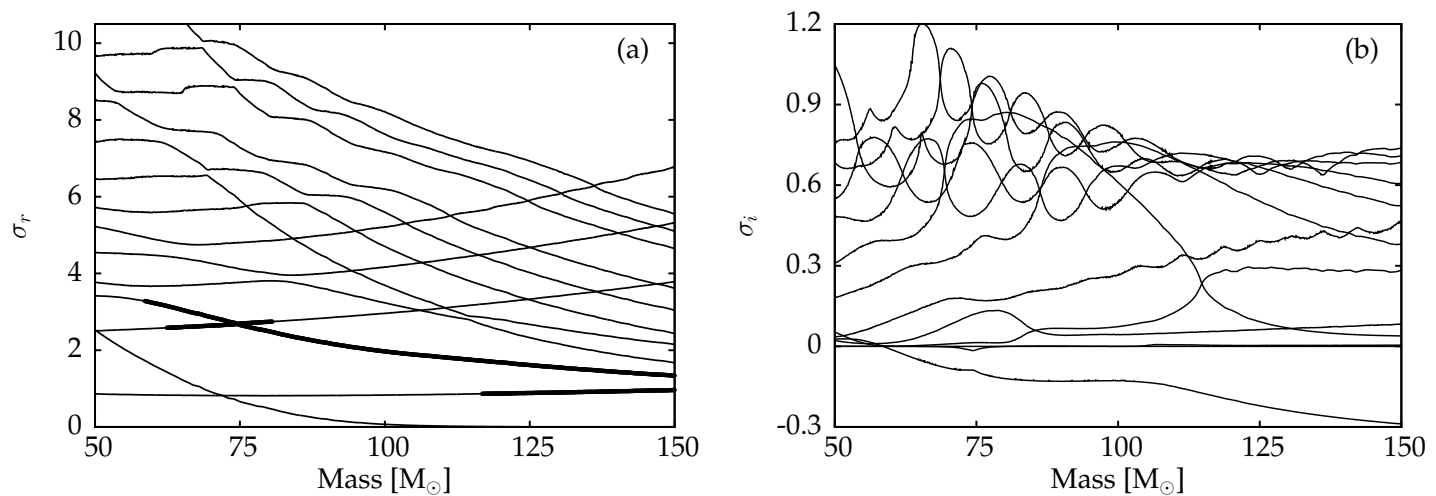

FIGURE 3.1: Real (a) and imaginary parts (b) of eigenfrequencies normalized by the global free fall time as a function of mass along a ZAMS with solar chemical composition. Unstable modes are represented by thick lines in (a) and by negative values of the imaginary part in (b).
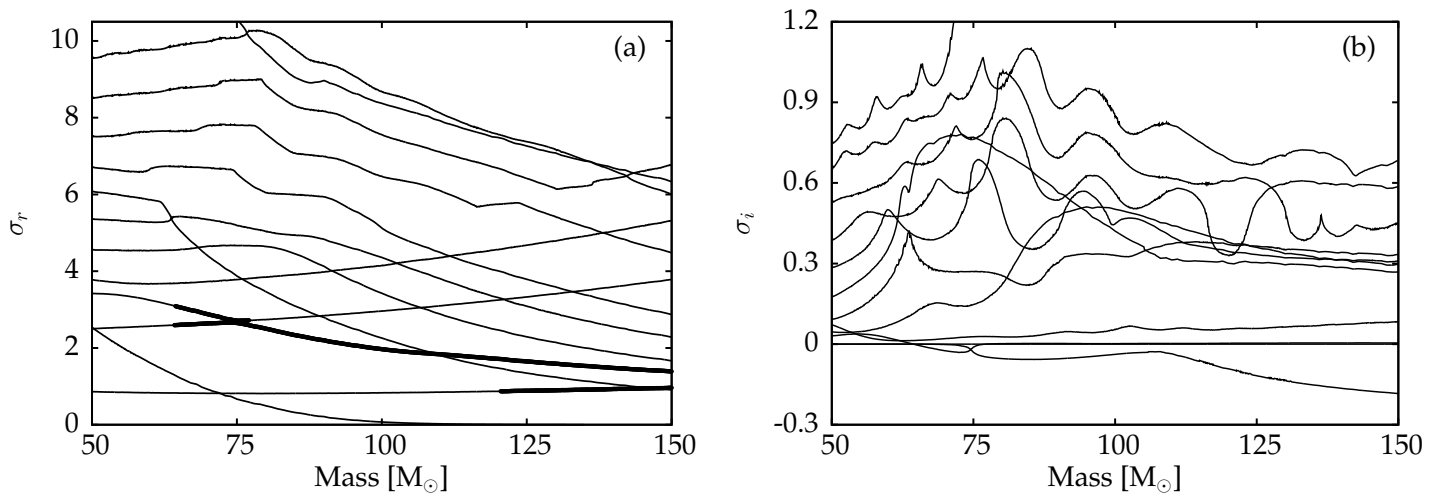

FIGURE 3.2: Same as Fig. 3.1 but for photospheric boundary conditions consistent with the previous study by Glatzel \& Kiriakidis (1993a).

(Goodman \& White, 2016) are highly questionable. Fig. 3.1 is based on photospheric boundary conditions consistent with the subsequent nonlinear simulations. In order to compare our results with previous studies by Glatzel \& Kiriakidis (1993a) the analysis has been redone using the same boundary conditions as these authors. The result is presented in Fig. 3.2. From a comparison of Figs. 3.1 and 3.2 we deduce that the basic modal structure and instabilities are not affected by the boundary conditions. Compared to Fig. 3.1 the onset of the instabilities is shifted towards slightly higher masses and the growth rates of the instabilities are reduced in Fig. 3.2. Comparing Fig. 3.2 with the previous study by Glatzel \& Kiriakidis (1993a) the basic modal structure and instabilities are identical. However, the onset of instabilities occurred at higher masses for the strange mode and at lower masses for the ordinary fundamental mode in the previous study. We suggest that updated opacities and equations of state are responsible for the small differences.

\subsection{Nonlinear simulations}

Our linear stability analysis has shown that the ZAMS models considered are violently unstable for masses above $58 \mathrm{M}_{\odot}$. To determine the final fate of the unstable models, 
the evolution of the instabilities has to be followed into the nonlinear regime. This is done using the numerical approach described in Grott et al. (2005). For the simulation of instabilities in stellar envelopes and of finite amplitude stellar pulsations a correct treatment of the energy balance of the system is crucial, since the dominant thermal and gravitational energies exceed the kinetic energy and the acoustic energy flux (being the quantities of interest) by several orders of magnitude. In the approach suggested by Grott et al. (2005) a highly accurate representation of the energy balance is achieved by use of an intrinsically conservative difference scheme. It is based on the Lagrangian description and turns out to be necessarily implicit with respect to time. For our nonlinear simulations, we have typically used 500 grid points, an iteration accuracy of $10^{-7}$ and an artificial viscosity parameter of 10 . For more details of the numerics, the reader is referred to Grott et al. (2005).

Once the acoustic energy flux at the outer boundary of a stellar model is determined by numerical simulations, the mass loss rate associated with a stellar wind possibly driven by the acoustic flux may be estimated. This is done (see Grott et al., 2005) by comparing the mean slope of the time integral of the acoustic luminosity with the kinetic luminosity $\frac{1}{2} \dot{M} v_{\infty}^{2}$ of the stellar wind, where $\dot{M}$ denotes the mass loss rate and $v_{\infty}$ stands for terminal velocity of the wind. The terminal velocity of the wind is estimated by the escape velocity from the object. Thus an estimate for the mass loss rate is obtained directly from the mean acoustic luminosity. Three models with 75, 90 and 150 $\mathrm{M}_{\odot}$ have been selected for nonlinear simulations.

\subsection{Results of nonlinear simulations for selected stellar models}

\subsection{1 $75 \mathrm{M}_{\odot}$}

According to the linear stability analysis the ZAMS model with $75 \mathrm{M}_{\odot}$ has two unstable modes with periods of 3.25 hours $\left(\sigma_{r}=2.65 ; \sigma_{i}=-0.094\right)$ and 3.20 hours $\left(\sigma_{r}=2.69\right.$; $\sigma_{i}=-0.012$ ). The evolution of the instabilities into the nonlinear regime is shown for this model in Fig. 3.3. Without any external perturbation the instability develops from numerical noise (of the order of $10^{-6} \mathrm{~cm} / \mathrm{s}$ in terms of the velocity), undergoes the linear phase of exponential growth and finally saturates in the nonlinear regime. In the linear phase, periods and growth rates may be compared with the results of the entirely independent linear stability analysis. Differences between the periods and the growth rates amount to less than one per cent. In the nonlinear regime, the velocity amplitude reaches values up to $10^{7} \mathrm{~cm} / \mathrm{s}$ thus remaining well below the escape velocity of the model. In the regime of stationary finite amplitude pulsations, the temperature at the outermost grid point varies between $19000 \mathrm{~K}$ and $31000 \mathrm{~K}$. We note that the position of the outermost grid point does not necessarily coincide with the photosphere. Therefore its temperature in general differs from the effective temperature. Together with temperature and density the variation of the bolometric magnitude exhibits a period of 0.14 day $(\approx 3.36$ hours $)$ in the finite amplitude pulsation regime. Thus compared to the linear phase periods have changed in the nonlinear regime by at most 10 per cent.

As discussed in the previous section, the correct treatment of the energy balance is essential. Fig. 3.3 illustrates that the dominant gravitational potential and internal energies exceed the kinetic energy and the time integrated acoustic flux by four orders of magnitude. This difference poses a challenging task for the numerical treatment. To prove that the scheme adopted satisfies the accuracy requirements and the simulations provide reliable results rather than numerical artifacts, the error in the energy balance is shown in Fig. 3.3 (i). It amounts to a fraction of $10^{-8}$ of the gravitational potential 

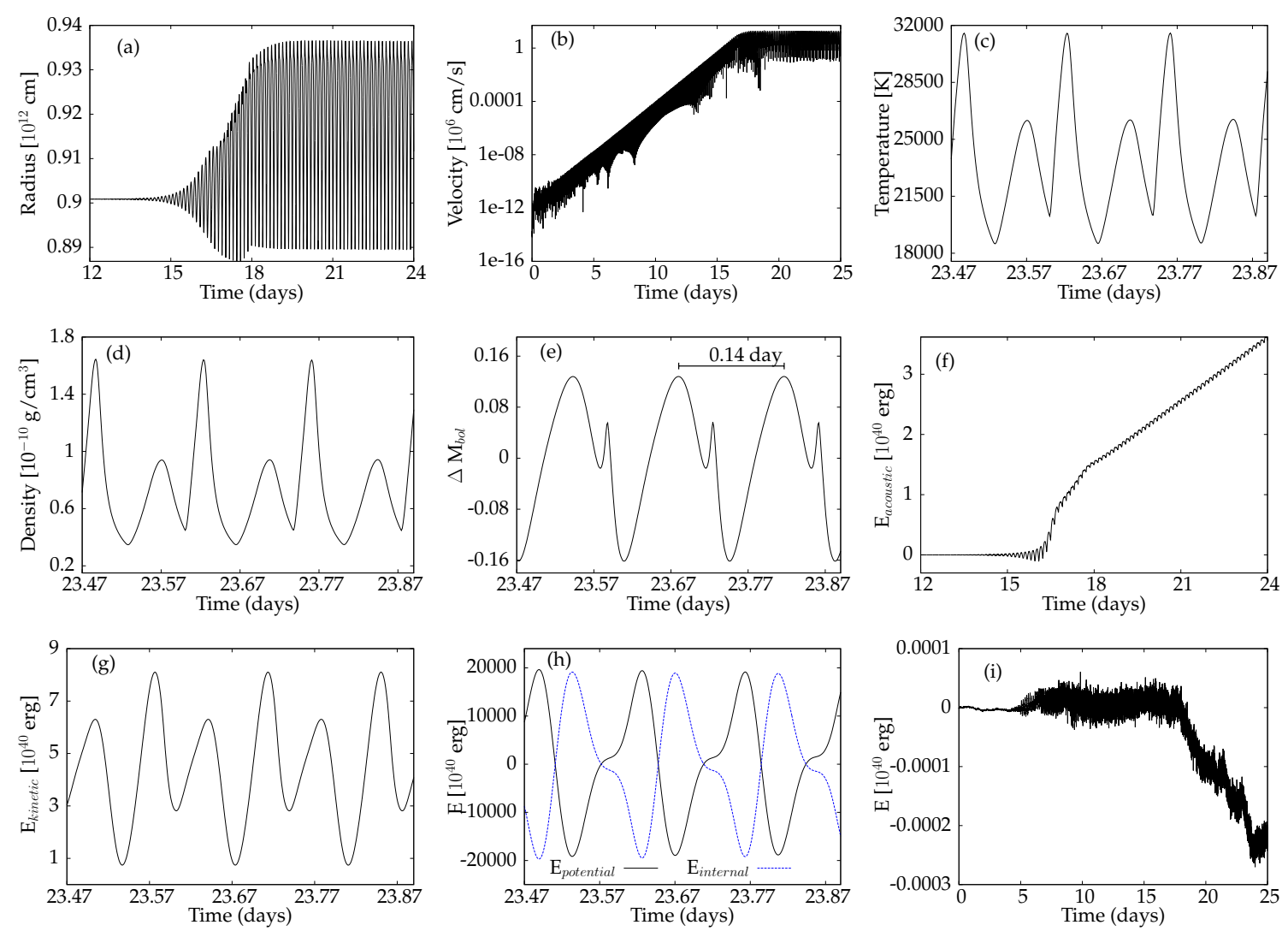

FIGURE 3.3: Evolution of instabilities and finite amplitude pulsations of a ZAMS model with $75 \mathrm{M}_{\odot}$ : Radius (a), velocity (b), temperature (c) and density (d) at the outermost grid point, the variation of the bolometric magnitude (e), the time integral of the acoustic luminosity (f), the kinetic energy $(\mathrm{g})$, the gravitational potential and internal energies $(\mathrm{h})$ and the error of the energy balance (i) as a function of time.

and the thermal energies (Fig. 3.3.h) and to a fraction of $10^{-4}$ of the kinetic energy and the time integrated acoustic flux. The time integrated acoustic luminosity is not a monotonic function (see Fig. 3.3.f) indicating that in each pulsation cycle the acoustic flux changes its sign. However, its mean over a pulsation cycle is positive implying that on average an outward acoustic flux is generated by the system. From Fig. 3.3.f we deduce that in the regime of stationary finite amplitude pulsations (after 18 days) the mean slope of the time integrated acoustic flux is constant. It corresponds to the mean acoustic luminosity of the pulsating star which is used to estimate the mass loss rate associated with a pulsationally driven wind in the way described in the previous section. Thus we obtain a mass loss rate of $0.59 \times 10^{-7} \mathrm{M}_{\odot} / \mathrm{yr}$ (see also Grott et al., 2005).

\subsection{2 $90 \mathrm{M}_{\odot}$}

For the $90 \mathrm{M}_{\odot}$ model, the linear stability analysis provides one low order unstable radial acoustic mode with a period of 4.38 hours $\left(\sigma_{r}=2.19 ; \sigma_{i}=-0.13\right)$. The results of our nonlinear simulations for this model are presented in Fig. 3.4. From Fig. 3.4 we deduce that even quantitatively they are similar to those discussed in the previous sections for the $75 \mathrm{M}_{\odot}$ model. Thus the discussion given there also refers to the $90 \mathrm{M}_{\odot}$ 

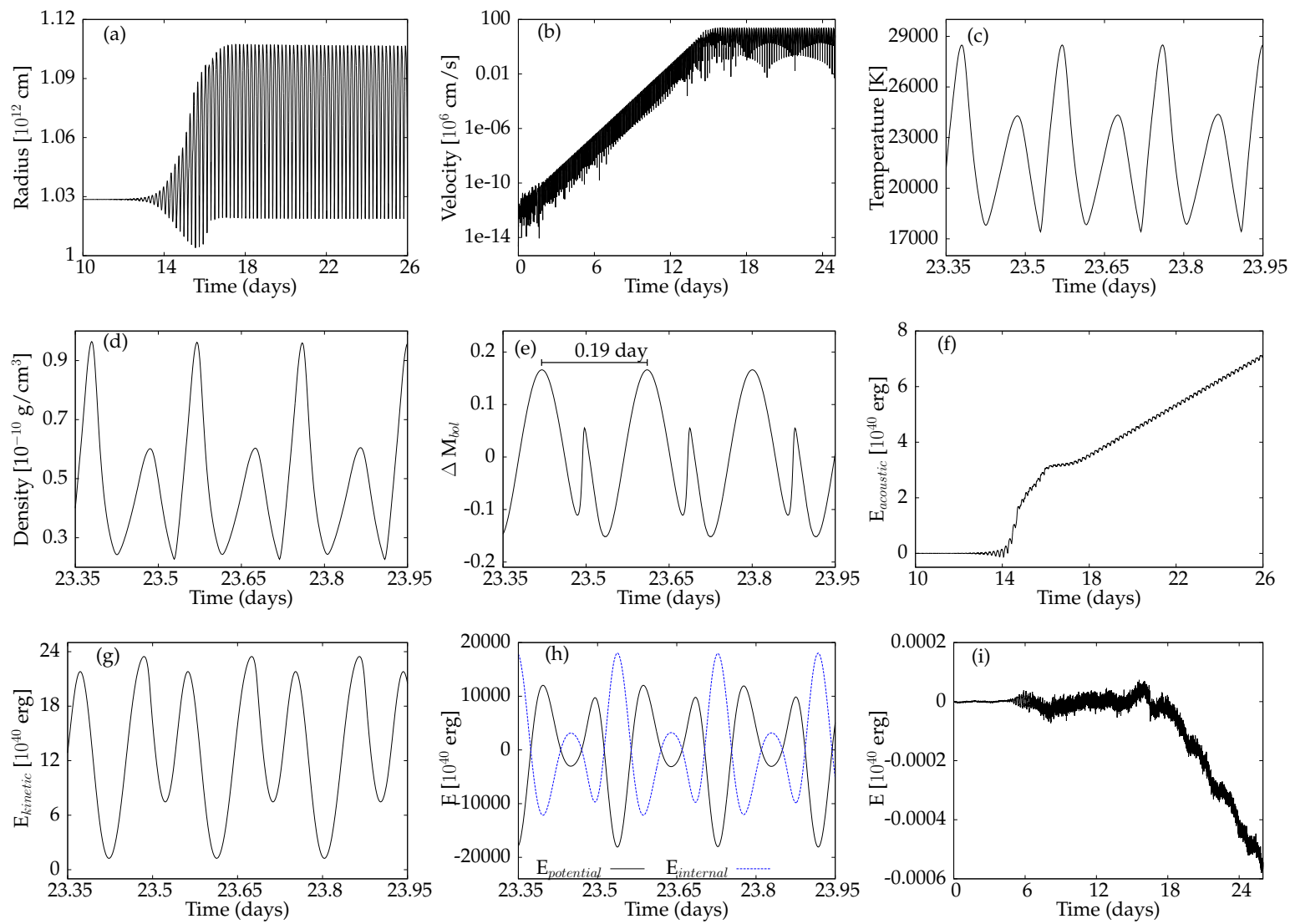

FIGURE 3.4: Same as Fig. 3.3 but for a ZAMS model with $90 \mathrm{M}_{\odot}$. 
model except for the finite amplitude pulsation period and the mass loss rate which now amount to 0.19 day (4.56 hours) and $0.69 \times 10^{-7} \mathrm{M}_{\odot} / \mathrm{yr}$, respectively.

\subsection{3 $150 \mathrm{M}_{\odot}$}

Similar to the $75 \mathrm{M}_{\odot}$ model, this model exhibits two unstable low order acoustic modes with periods of 11.5 hours $\left(\sigma_{r}=1.33, \quad \sigma_{i}=-0.288\right)$ and 15.9 hours $\left(\sigma_{r}=0.96, \quad \sigma_{i}=\right.$ $-0.17 \times 10^{-5}$ ), respectively. The results obtained by following the instabilities into the nonlinear regime are shown in Fig. 3.5. Compared to the previously discussed models with 75 and $90 \mathrm{M}_{\odot}$ they are qualitatively similar and the discussions presented there also refer to the $150 \mathrm{M}_{\odot}$ model except for the finite amplitude pulsation period and the mass loss rate which now amount to 0.99 day (23.76 hours) and $0.25 \times 10^{-7} \mathrm{M}_{\odot} / \mathrm{yr}$, respectively. Contrary to the 75 and $90 \mathrm{M}_{\odot}$ models, the finite amplitude pulsation period of 23.76 hours is substantially longer than the initial pulsation period of 11.5 hours in the linear regime of the evolution of the instability (see table 3.2). The difference is a nonlinear effect and caused by a considerable inflation of the envelope by the instability. This can be deduced from Fig. 3.5(a) where the radius is found to increase from its initial hydrostatic value of $1.65 \times 10^{12} \mathrm{~cm}$ to a mean value of $2 \times 10^{12} \mathrm{~cm}$ in the nonlinear regime. An increased radius implies a lower mean density and, according to the period-density relation, a longer period.
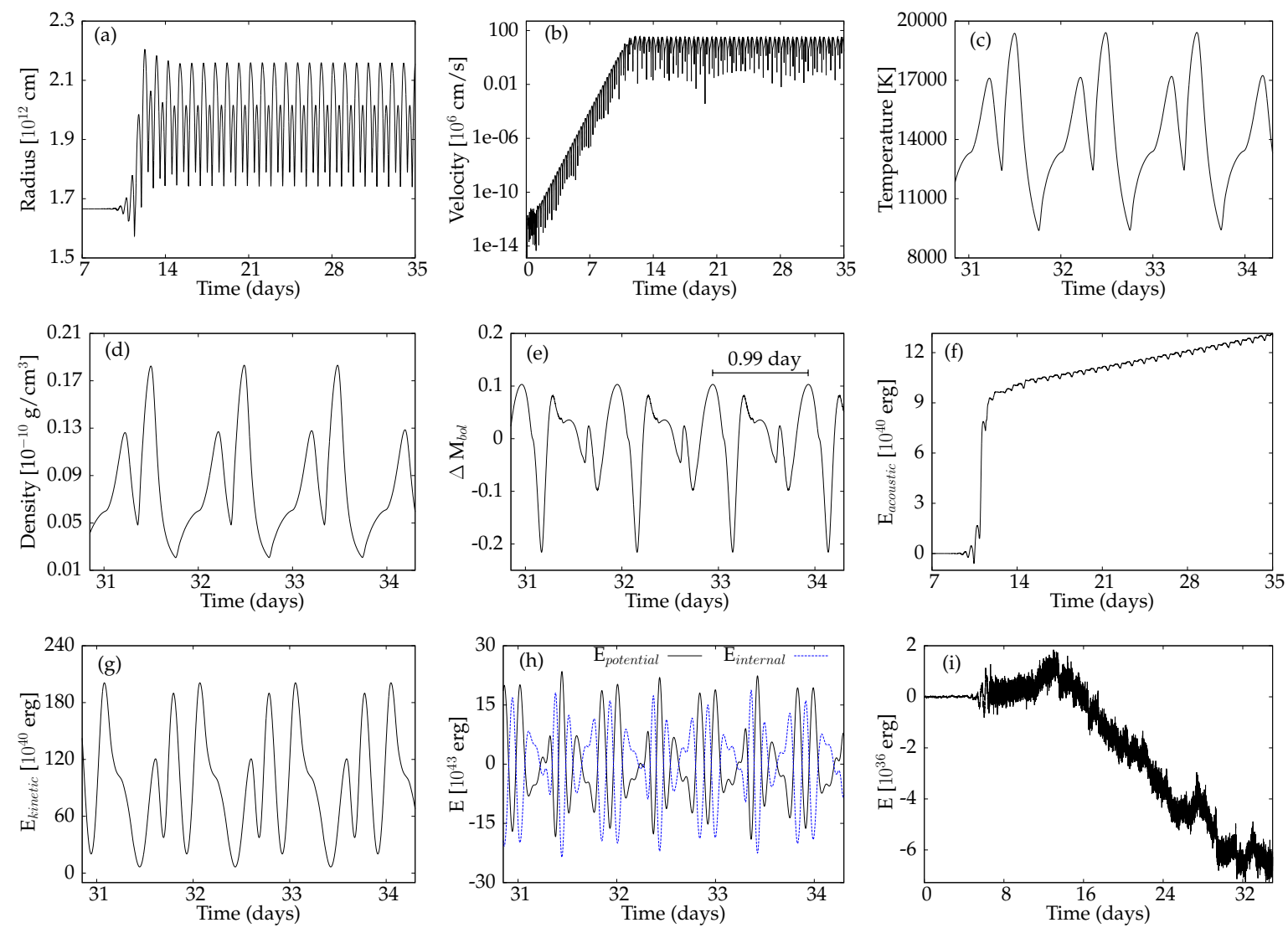

FIGURE 3.5: Same as Fig. 3.3 but for a ZAMS model with $150 \mathrm{M}_{\odot}$.

Thus, for very massive ZAMS stars the final finite amplitude pulsation periods may significantly differ from periods determined by a linear analysis. As a consequence, observed periods should not be compared to the latter but to periods determined by 
TABLE 3.2: Pulsation periods and mass loss rates for the ZAMS models selected.

\begin{tabular}{cccc}
\hline $\begin{array}{c}\text { Mass } \\
\left(\mathrm{M}_{\odot}\right)\end{array}$ & $\begin{array}{c}\text { Linear Period } \\
\text { (hours) }\end{array}$ & $\begin{array}{c}\text { Nonlinear Period } \\
\text { (hours) }\end{array}$ & $\begin{array}{c}\text { Mass Loss Rate } \\
\left(\mathrm{M}_{\odot} / \mathrm{yr}\right)\end{array}$ \\
\hline 75 & 3.25 & 3.36 & $0.59 \times 10^{-7}$ \\
90 & 4.38 & 4.56 & $0.69 \times 10^{-7}$ \\
150 & 11.50 & 23.76 & $0.25 \times 10^{-7}$ \\
\hline
\end{tabular}

nonlinear simulations. These findings are in agreement with previous investigations of models for 55 Cygni (Yadav \& Glatzel, 2016). These authors find substantial differences between linear and nonlinear periods and emphasize that only nonlinear periods should be compared with observations, in particular for strong instabilities such as strange mode instabilities.

\subsection{Discussion and conclusions}

We have examined the stability of massive zero age main sequence models with solar chemical composition in the mass range between 50 and $150 \mathrm{M}_{\odot}$ with respect to infinitesimal radial perturbations. The linear stability analysis shows that higher order modes are stable and hence not of interest in the present context. Instabilities, in particular strong strange mode instabilities, are associated with low order modes for models having masses above a critical mass of $58 \mathrm{M}_{\odot}$. The critical mass for instability of ZAMS stars has a long history and was investigated by many authors (see e.g., Fadeyev, 2011; Glatzel \& Kiriakidis, 1993a; Papaloizou, 1973a,b; Schwarzschild \& Härm, 1959; Shiode et al., 2012; Stothers, 1992; Ziebarth, 1970). It depends sensitively on chemical composition and opacity. For solar chemical composition, Fadeyev (2011) suggested that models having masses above $50 \mathrm{M}_{\odot}$ are unstable, while Glatzel \& Kiriakidis (1993a) find a critical mass of $80 \mathrm{M}_{\odot}$. In early investigations $\varepsilon$ - instability of the fundamental mode was considered to be responsible for the instability of main sequence stars. Although present, it is now considered to be of academic interest only, since its growth timescale competes with the stellar evolution timescale and simultaneously present strange mode instabilities grow on the dynamical timescale.

In this context, Goodman \& White (2016) have recently studied instabilities associated with the fundamental mode in models of very massive metal rich stars. They concluded that the growth rate of the fundamental mode is too small to limit the lifetime of very massive stars on the main sequence. These findings are in accordance with Glatzel \& Kiriakidis (1993a) who claimed that the growth rate of the fundamental mode is extremely small compared to that of other unstable modes and therefore its instability is of minor importance.

To determine the final fate of unstable models, we have followed the instabilities into the nonlinear regime by numerical simulations. Their results for three selected models having masses of 75,90 and $150 \mathrm{M}_{\odot}$, respectively, have been discussed in this chapter. The nonlinear simulations of the instabilities lead to finite amplitude pulsations with basic periods in the range between 3 hours and 1 day. Periods determined by the linear stability analysis are close to the nonlinear finite amplitude periods in less massive models $\left(<90 \mathrm{M}_{\odot}\right)$. However, the finite amplitude period of the most massive $\left(150 \mathrm{M}_{\odot}\right)$ model considered significantly differs from the linearly determined period 
due to a substantial inflation of the envelope. Therefore in such cases, pulsation periods predicted by a linear stability analysis should not be compared with observed periods. Rather nonlinear simulations need to be done and their results in terms of finite amplitude periods have to be used for comparison.

Mass loss plays an important role in the evolution of massive stars. Meanwhile there is growing evidence for a connection between mass loss and pulsations in massive stars (see e.g., Glatzel et al., 1999; Kraus et al., 2015; Neilson, 2014; Yadav \& Glatzel, 2016). On the basis of our nonlinear simulations for ZAMS models, we have estimated the mass loss rate associated with a pulsationally driven stellar wind to lie in the range between $0.25 \times 10^{-7}$ and $0.69 \times 10^{-7} \mathrm{M}_{\odot} / \mathrm{yr}$ (see table 3.2). Thus pulsationally driven winds and associated mass loss rates may have an influence on stellar evolution.

The present study has been restricted to considering radial perturbations. A linear stability analysis with respect to nonradial perturbations of the models considered here will be presented elsewhere. The simulation of the evolution of nonradial instabilities into the nonlinear regime, however, is not yet feasible. 


\section{Chapter 4}

\section{Stability analysis and nonlinear pulsations of 55 Cygni (HD 198478) $)^{1}$}

\subsection{Introduction}

Supergiants play a major role for any galaxy because these massive stars are responsible for the chemical enrichment (Matteucci, 2008; Nomoto et al., 2013). According to Puls et al. (2008), massive stars are 'critical agents' in galactic evolution. Most of the heavy elements are produced in massive stars and injected into the interstellar medium by mass loss due to stellar winds or explosions of stars. Stellar mass loss is not only crucial for galactic evolution but is also important for stellar evolution. In particular, it determines the final fate of a massive star (Smith, 2014). Apart from mass loss, pulsation is a common phenomenon in supergiants, where both radial and nonradial modes have been identified. In addition to ordinary pressure and gravity modes, the presence of strange modes (see, e.g. Gautschy, 1992; Gautschy \& Glatzel, 1990b; Saio \& Jeffery, 1988; Wood, 1976) and associated instabilities was theoretically predicted by Kiriakidis et al. (1993) and seems to be confirmed in the supergiants HD 50064 (Aerts et al., 2010b) and 55 Cygni (Kraus et al., 2015). In classical pulsators pulsations are usually excited by the $\kappa$ - mechanism. For example, slowly pulsating B stars (SPB) pulsations are driven by the $\kappa$-mechanism due to the Fe-opacity bump (Pamyatnykh, 1999). Contrary to the classical pulsators, instabilities associated with strange modes are not excited by the $\kappa$ - mechanism (Gautschy \& Glatzel, 1990b). For a model of the strange mode instability mechanism see Glatzel (1994). Maeder (1980) found a substantial increase in mass loss with the amplitude of light variations in B and A type supergiants. Glatzel et al. (1999) pointed out that strange modes instability can lead to a pulsationally driven mass loss phenomenon in massive stars. However, despite many investigations (e.g., Kambe et al., 1993; Maeder, 1980) the connection between pulsations and mass loss is still a matter of debate.

Motivated by recent observations (Kraus et al., 2015), we shall present in this paper a theoretical study of both the linear stability and the nonlinear evolution of strange mode instabilities in models for supergiants. With respect to the observations, we restrict our study to models for 55 Cygni (HD 198478) - a B-type supergiant situated in the galactic bar (Jurkić et al., 2011). This star exhibits photospheric as well as spectroscopic variability. A P-Cygni profile in the $\mathrm{H}_{\alpha}$ line indicates mass loss. Maharramov (2013) states that the $\mathrm{H}_{\alpha}$ line is variable and sometimes even completely vanishes. Maharramov (2013) also reports the inversion of the P-Cygni profile of $\mathrm{H}_{\alpha}$ and interprets it as an indication for high velocity material in a stellar wind. In a recent study, Kraus et al. (2015) points out that 55 Cygni pulsates in pressure, gravity and strange modes. The pulsation periods lie in the range between 2.7 hours and 22.5 days. These authors

\footnotetext{
${ }^{1}$ An earlier version of this chapter has been published in MNRAS (Yadav \& Glatzel, 2016).
} 
also claim that the pulsations of 55 Cygni are associated with episodes of enhanced mass loss and derive an estimate for the mass loss rate.

Thus motivated, we shall perform a linear stability analysis as well as nonlinear simulations for stellar models with parameters close to that of 55 Cygni. The construction of stellar models and their linear stability analysis is described in sections 4.2 and 4.3 , respectively. Results of the linear stability analysis are discussed in section 4.4, simulations of the evolution of instabilities into the nonlinear regime are presented for selected models in section 4.5. Our conclusions follow.

\subsection{Models}

We have constructed envelope models for masses between 14 and $40 \mathrm{M}_{\odot}$ with solar chemical composition. In order to investigate the effect of chemical composition, we have also constructed envelope models for the same mass range $\left(14-40 \mathrm{M}_{\odot}\right)$ with $X$ $=0.20, \mathrm{Y}=0.78$ and $\mathrm{Z}=0.02$. Where $\mathrm{X}, \mathrm{Y}$ and $\mathrm{Z}$ represent the mass fraction of $\mathrm{H}, \mathrm{He}$ and metals respectively. A stability analysis of enhanced He or H-deficient models is motivated by the fact that Markova \& Puls (2008) and Underhill (1969) suggested 55 Cygni to be a He-rich / H-deficient star. We adopt an effective temperature of $18800 \mathrm{~K}$ and a luminosity of $\log L / L_{\odot}=5.57$ for our models according to the recent study of 55 Cygni by Kraus et al. (2015).

The envelope models are based on the OPAL opacity tables (Iglesias \& Rogers, 1996; Rogers \& Iglesias, 1992; Rogers et al., 1996). Convection is treated according to standard mixing length theory (Böhm-Vitense, 1958) with 1.5 pressure scale heights for the mixing length. Magnetic fields and rotation are disregarded for simplicity.

\subsection{Linear stability analysis}

A linear stability analysis with respect to radial perturbations is performed on the basis of the pulsation equations expressed in the form given by Gautschy \& Glatzel (1990b). The equations are solved using the Riccati method as described by Gautschy \& Glatzel (1990a). As the interaction of pulsation with convection is still poorly understood, we have used here the 'frozen in approximation' (Baker \& Kippenhahn, 1965) for convection. It consists of neglecting the Lagrangian perturbation of the convective flux and holds if the timescale of pulsation is short compared to the timescale of convection. Moreover, if the contribution of the convective flux to the total flux is small, the effect of the details of treatment of convection is expected to be negligible. In fact, these conditions are met in the models considered.

The pulsation equations form an eigenvalue problem with complex eigenfrequencies and eigenfunctions. In our analysis, the eigenfrequencies $(\sigma)$ are normalized with the global free fall time $\tau_{\mathrm{ff}}=\sqrt{R^{3} / 3 G M}$ where $R$ is the stellar radius, $G$ stands for the gravitational constant, and $M$ denotes the stellar mass. Real parts $\left(\sigma_{\mathrm{r}}\right)$ of the eigenfrequency represent the pulsation period while imaginary parts provide information about damping or excitation. In the normalization adopted, negative imaginary parts $\left(\sigma_{\mathrm{i}}<0\right)$ correspond to unstable modes, positive values $\left(\sigma_{\mathrm{i}}>0\right)$ to damped modes. 


\subsection{Results of the linear stability analysis}

We have performed a linear stability analysis for masses between 14 and $40 \mathrm{M}_{\odot}$ both with solar and He enhanced chemical compositions. The analysis is based on two series of 261 envelope models which were constructed by keeping the effective temperature as well as the luminosity constant and varying the mass. A sufficiently small stepsize in mass (we used $0.1 \mathrm{M}_{\odot}$ ) guarantees an acceptably smooth variation of the eigenfrequencies with mass. The representation of eigenfrequencies as a function of a stellar parameter (preferably stellar mass or effective temperature) is generally denoted as a "modal diagram" (Saio et al., 1998).
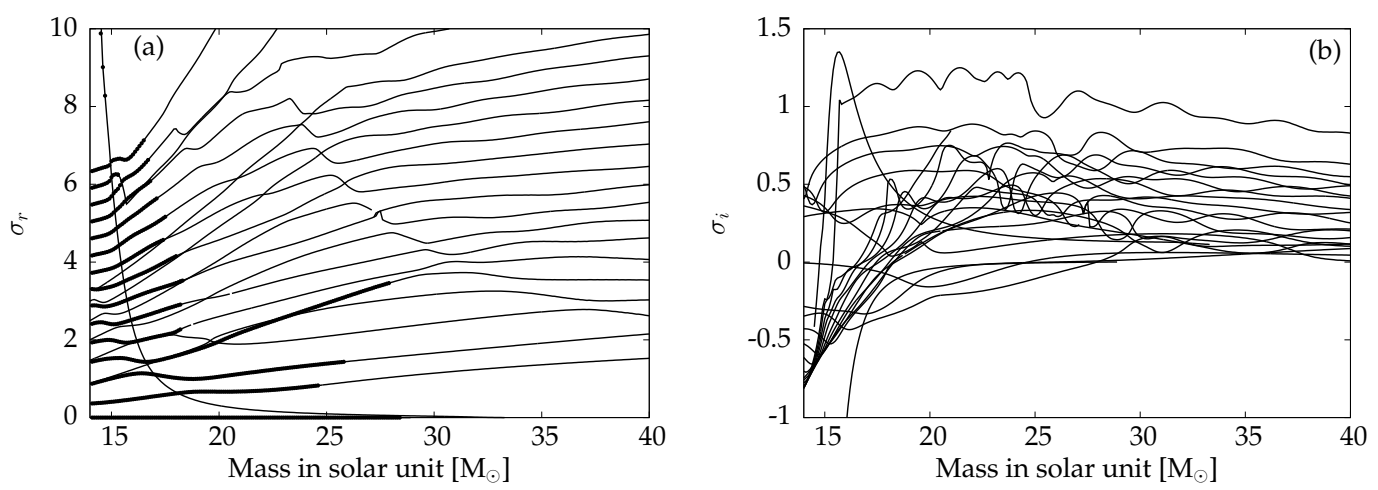

FIGURE 4.1: Real (a) and imaginary (b) parts of the eigenfrequencies normalized to free fall time as a function of mass for models of 55 Cygni with luminosity $\log L / L_{\odot}=5.57$, effective temperature $18800 \mathrm{~K}$ and solar chemical composition $(X=0.70, Y=0.28, Z=0.02)$. Thick lines in (a) and negative imaginary parts in (b) correspond to unstable modes.

Figs. 4.1 and 4.2 are the modal diagrams for solar and He enhanced compositions respectively. Figs. 4.1(a) and 4.2(a) represent the real parts of the lowest order eigenfrequencies as a function of stellar mass, where thick lines denote unstable modes. These correspond to negative imaginary parts $\left(\sigma_{\mathrm{i}}<0\right.$; see section 4.3 for details) in Figs. 4.1(b) and 4.2(b). Both with respect to the range of unstable models and the strength of instabilities, the restriction to the low order modes shown seems to be sufficient. The structure of modes indicates a variety of mode couplings in terms of avoided crossings and instability bands which is typical for the strange mode phenomenon. Thus
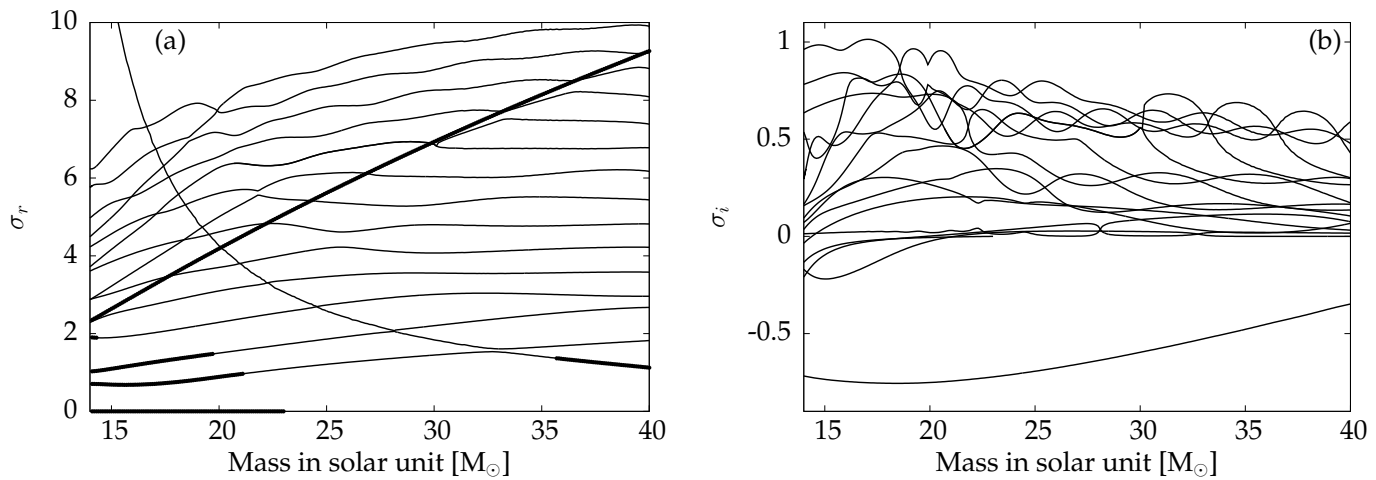

FIGURE 4.2: Same as Fig. 4.1 but for enhanced He abundance $(\mathrm{Y}=0.78$,

$$
\mathrm{X}=0.20) \text {. }
$$



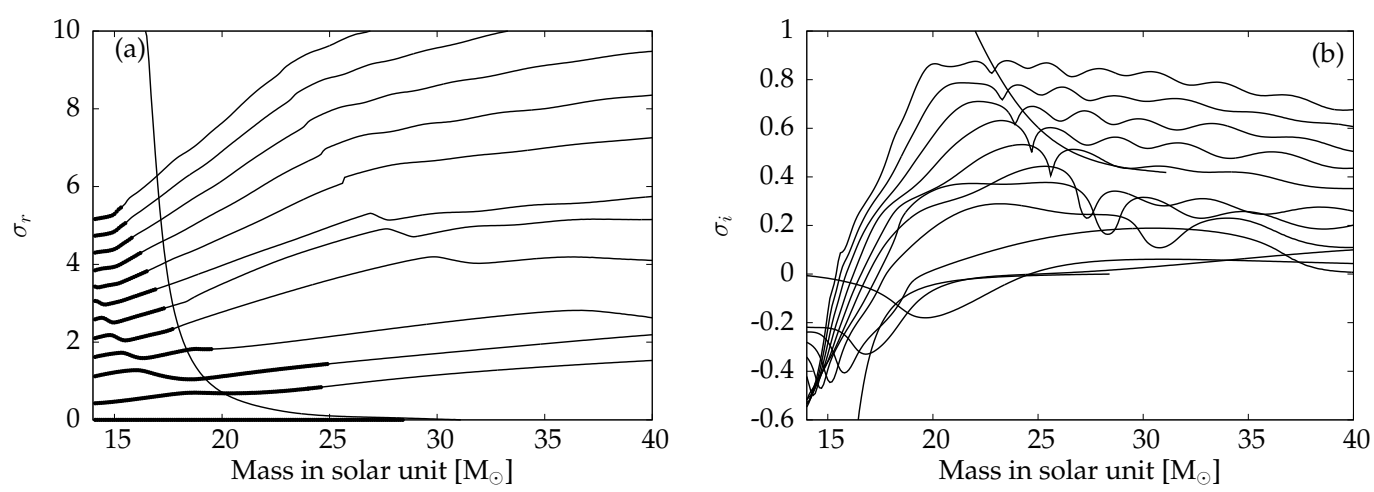

FIGURE 4.3: Same as Fig. 4.1 but for different outer boundary conditions
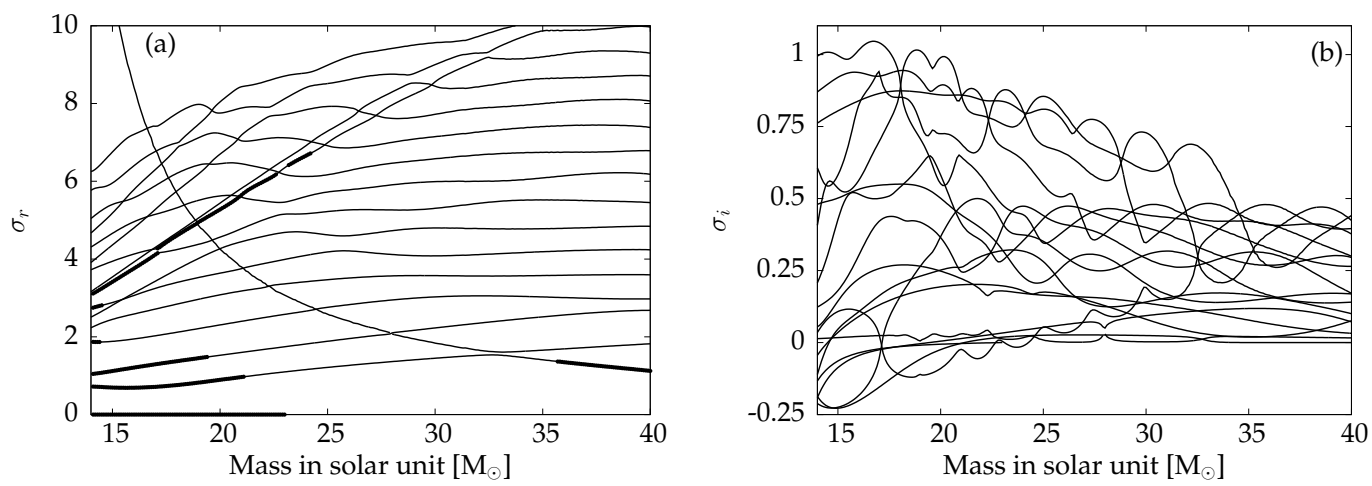

FIGURE 4.4: Same as Fig. 4.3 but for enhanced He abundance $(Y=0.78$, $\mathrm{X}=0.20)$.

we may address the instabilities found as strange mode instabilities. The strange mode phenomenon becomes more pronounced with decreasing mass. Accordingly the spacing of pulsation frequencies is still regular at high masses and becomes more and more irregular for smaller masses (or as $L / M$ increases).

From Fig. 4.1, we deduce that many modes are unstable in models having masses less than $20 \mathrm{M}_{\odot}$, but no unstable mode is found in models with masses above $28 \mathrm{M}_{\odot}$. The growth rates of unstable modes associated with the imaginary parts of the eigenfrequencies (Fig. 4.1.b) in general decrease with mass. We note the existence of a monotonically $\left(\sigma_{\mathrm{r}}=0\right)$ unstable mode (Deller, 2009; Hilker, 2009; Saio, 2011, Yadav \& Glatzel in prep.). It dominates for masses below $\approx 17 \mathrm{M}_{\odot}$, but does not determine the stability boundary at $28 \mathrm{M}_{\odot}$.

Compared to solar composition, models with enhanced He abundance (Fig. 4.2) exhibit a qualitatively similar behaviour. However, instabilities do no longer persist to higher order modes for small masses. The lowest order modes become unstable below $22 \mathrm{M}_{\odot}$. A crossing mode, whose frequency decreases with mass, now becomes unstable for masses above $35 \mathrm{M}_{\odot}$. The most unstable mode over the entire mass interval crosses the ordinary spectrum. Its frequency increases with mass. A much less unstable counterpart may be identified also in the spectrum of the solar composition models. Compared to solar composition the monotonically unstable mode has smaller growth rates.

The outer boundary conditions for the perturbation equations are ambiguous, because the outer boundary of the stellar model does not coincide with the physical 
boundary for the star. Figs. 4.1 and 4.2 are for consistency based on the same conditions which have been adopted in the subsequent nonlinear simulations: In order to avoid reflections of sound waves and shocks at the outer boundary the divergence of the heat flux and the gradient of the divergence of the velocity are required to vanish there (Grott et al., 2005). Whether the linear stability analysis depends on the boundary conditions needs to be tested. Therefore we have repeated the linear analysis with boundary conditions requiring the Lagrangian pressure perturbation to vanish and StefanBoltzmann's law to hold at the outer boundary. The results are shown in Figs. 4.3 and 4.4 for solar composition and enhanced He abundance respectively. Apart from the fact, that the most unstable mode present in Fig. 4.2 has become less unstable or even stable in Fig. 4.4, a comparison of Fig. 4.1 with 4.3 and Fig. 4.2 with 4.4 exhibits a similar behaviour. An influence of the boundary conditions on the eigenfrequency is expected, if the amplitude of the eigenfunction of the mode considered is significant at the boundary. Thus we conclude that the dominant unstable modes in the He enhanced models have their maximum amplitudes close to the outer boundary.

\subsection{Nonlinear simulations}

In order to determine the final fate of linearly unstable stellar models, the instabilities need to be followed into the nonlinear regime by numerical simulation. For selected unstable models, we have performed such simulations on the basis of the procedure described by Grott et al. (2005). Starting from an initial unstable model in hydrostatic equilibrium the instability develops from numerical noise, undergoes the linear phase of exponential growth and saturates in the nonlinear regime. For details of the numerics such as grid resolution, artificial viscosity and iteration accuracy, we refer to Grott et al. (2005). In the simulations discussed here we used 500 grid points, an iteration accuracy of $10^{-7}$, and the artificial viscosity parameter was set to 10 . As shown by Grott et al. (2005), the simulations in the nonlinear regime may provide the acoustic energy lost from the system through its outer boundary as a function of time. Being a nonmonotonic function the slope of its increasing mean corresponds to the mean acoustic luminosity of the object. Assuming that this acoustic luminosity $L_{a c}$ is the origin of a stellar wind, the mass loss rate $\dot{M}$ associated with it may be estimated by comparing the kinetic luminosity of the wind $\frac{1}{2} \dot{M} v_{\infty}^{2}$, where $v_{\infty}$ is the terminal wind velocity, to the numerically determined acoustic luminosity:

$$
\frac{1}{2} \dot{M} v_{\infty}^{2} \approx L_{a c} \Longrightarrow \dot{M} \approx \frac{2 \times L_{a c}}{v_{\infty}^{2}}
$$

For our estimates of mass loss rates we assume the terminal wind velocity to be of the order of the escape velocity of the models considered.

The correct energy balance and, associated with it, the proper treatment of the different forms of the energies is crucial for the quality and reliability of the results, which can be seen by considering the various forms of the energy involved in stellar pulsations (see Grott et al., 2005). Gravitational potential and thermal energies are typically more than two orders of magnitude bigger than the kinetic energies which control the final velocity amplitudes to be determined by the simulations. The time integrated acoustic fluxes used for the determination of mass loss rates are even smaller by three orders of magnitude than the maximum energies. Thus the relative error in the energy balance (compared to the maximum energies) has to be smaller than at least $10^{-4}$ for the velocity amplitudes and mass loss rates determined to be reliable. How relative errors 
below $10^{-7}$ can be achieved by a fully conservative numerical scheme is described in Grott et al. (2005). In order to prove the reliability of our simulations we shall provide also various energies and the error of the energy balance below.

The linear analysis discussed above has provided the mass range of unstable stellar models: for solar chemical composition models with masses below $28 \mathrm{M}_{\odot}$ are unstable, He enhanced models are unstable over the entire mass range studied (14 to $40 \mathrm{M}_{\odot}$ ). From observations the following estimates of the mass of 55 Cygni are available:

1. Kraus et al. (2015) : $34 \pm 4 \mathrm{M}_{\odot}$

2. Gies \& Lambert (1992) : $32.1 \mathrm{M}_{\odot}$

3. Searle et al. (2008) : $23 \mathrm{M}_{\odot}$

4. Markova \& Puls (2008) : $11_{-3}^{+5} \mathrm{M}_{\odot}$

According to the various observational determinations of the mass of 55 Cygni and the results of the linear stability analysis, we have chosen to consider for nonlinear simulations two models with solar composition having masses of 23 and $25 \mathrm{M}_{\odot}$. In addition to solar composition models, three models with enhanced He abundance having masses 30, 34 and $17 \mathrm{M}_{\odot}$ are also included for nonlinear simulations.

\subsubsection{Models with solar composition}

$23 \mathbf{M}_{\odot}$

The linear stability analysis of the model with a mass of $23 \mathrm{M}_{\odot}$ reveals a most unstable, dominant mode with a period of 2.4 days and a growth of 0.18 /day. With a free fall time of $\tau_{\mathrm{ff}}=83740 \mathrm{~s}$ for this model they correspond to $\sigma_{\mathrm{r}}=2.57$ and $\sigma_{\mathrm{i}}=-0.173$ in normalized form. The additional less unstable modes do not significantly influence the result of the simulations. The results of our simulation of the evolution of this instability from hydrostatic equilibrium into the nonlinear regime is shown in Fig. 4.5. The simulation starts from numerical noise at a level of $10^{-4} \mathrm{~cm} / \mathrm{s}$ in terms of the velocity of the outermost grid point (see Fig. 4.5.b ). The code then picks up the most unstable mode with the independently previously determined linear period and growth rate (linear phase of exponential growth). Once the amplitude of the velocity has reached $10^{4} \mathrm{~cm} / \mathrm{s}$, deviations from linear behaviour appear, and at a level of $77 \mathrm{~km} / \mathrm{s}$ nonlinear saturation is established. It is characterized by a stationary finite amplitude pulsation with a period of 7.5 days (see Fig. 4.5.d for the variation of the bolometric luminosity). The increase of the pulsation period from 2.4 days in the linear phase to 7.5 days in the final nonlinear regime is essentially caused by the nonlinear inflation of the mean stellar radius (see Fig. 4.5.a). Associated with the increase of the radius is a significant decrease of the surface temperature (temperature of the outermost grid point) of the model (see Fig. 4.5.c ). Gravitational potential, internal and kinetic energies as a function of time (stationary initial values are subtracted) are shown in Figs. 4.5(h) and (e). These figures exhibit the behaviour typical for stellar pulsations: being of the same order of magnitude potential and internal energies exceed the kinetic energy by at least two orders of magnitude. The acoustic energy lost by the system (equivalent to the time integrated acoustic luminosity) as a function of time is displayed in Fig. 4.5(f). It is smaller than the kinetic energy by one order of magnitude and increases non monotonically with time. The non monotonic run means that within one pulsation cycle we have phases of incoming and outgoing acoustic fluxes, where the time integral over 

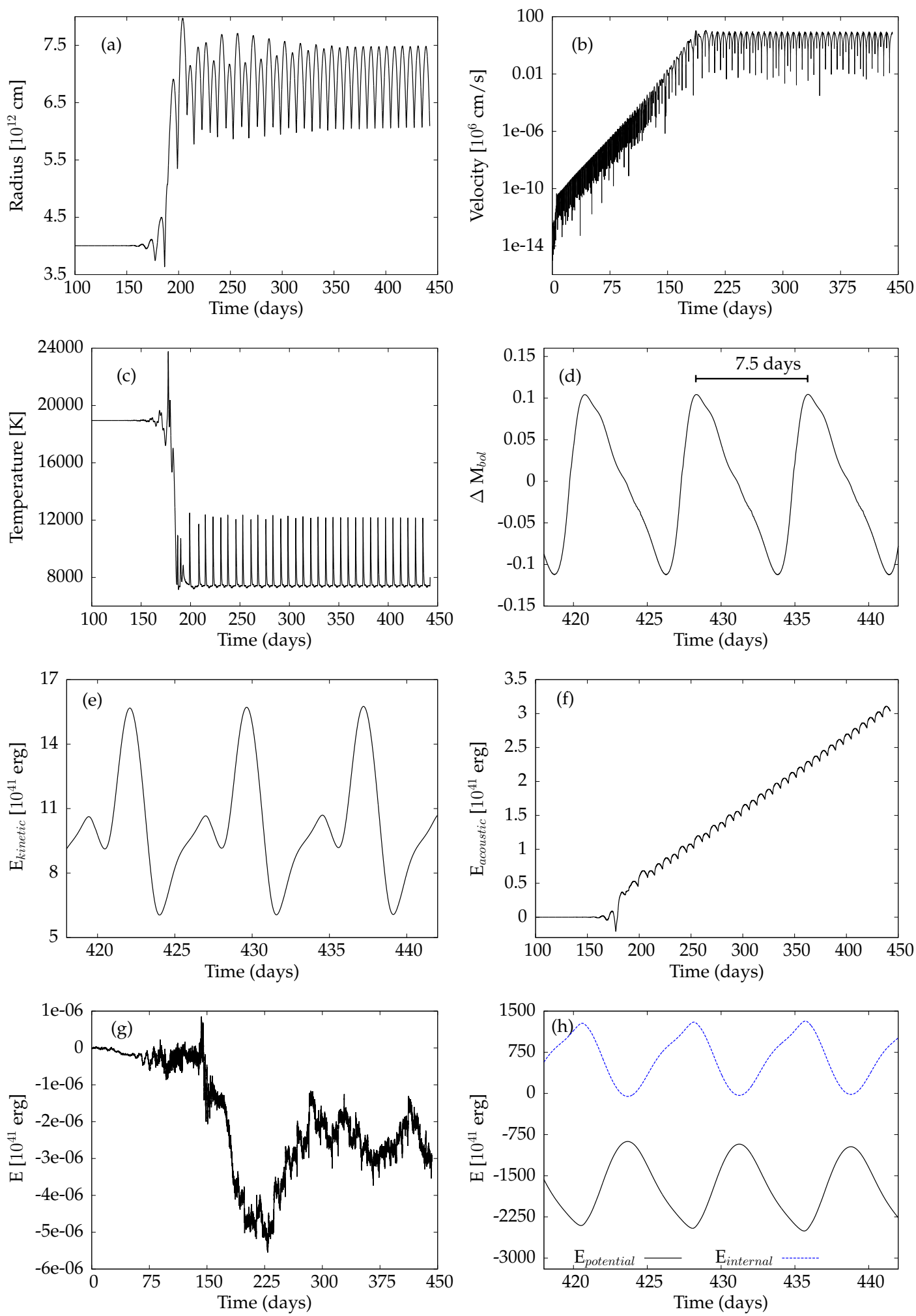

FIGURE 4.5: The evolution of a $23 \mathrm{M}_{\odot}$ model with solar chemical composition. The following quantities are given as a function of time: Radius (a), velocity (b) and temperature (c) of the outermost grid point, variation of the bolometric magnitude (d) and kinetic (e), internal and gravitational potential $(\mathrm{h})$ energies for a selected time interval, the timeintegrated acoustic luminosity (f) and the error in the energy balance (g). 
the outgoing flux exceeds the integral over the incoming flux. The mean slope of the function shown in Fig. 4.5(f) then corresponds to the mean acoustic luminosity of the system. As described above on the basis of this mean acoustic luminosity the mass loss of the model is estimated to amount to $2.6 \times 10^{-7} \mathrm{M}_{\odot} / \mathrm{yr}$. Finally the error in the energy balance as a function of time is given in Fig. 4.5(g). It is still six orders of magnitude smaller than the smallest contribution to the energy balance (the time integrated acoustic luminosity) which guarantees a meaningful interpretation of the results of the simulation.

$25 \mathbf{M}_{\odot}$

Similar to the $23 \mathrm{M}_{\odot}$ model, the linear stability analysis of the model with a mass of 25 $\mathrm{M}_{\odot}$ reveals a most unstable, dominant mode with a period of 1.98 days and a growth rate of $0.12 /$ day. With a free fall time of $\tau_{\mathrm{ff}}=80330 \mathrm{~s}$ for this model they correspond to $\sigma_{\mathrm{r}}=2.94$ and $\sigma_{\mathrm{i}}=-0.108$ in normalized form. The result of our simulation of the evolution of this instability from hydrostatic equilibrium into the nonlinear regime is qualitatively similar to the previous simulation of the $23 \mathrm{M}_{\odot}$ model and shown in Fig. 4.6 to be compared with its counterpart Fig. 4.5. The final nonlinear velocity amplitude reaches $108 \mathrm{~km} / \mathrm{s}$ which amounts to $27 \%$ of the escape velocity $(406 \mathrm{~km} / \mathrm{s})$ from the object. The nonlinear inflation of the envelope increases the pulsation period to 7.8 days in the nonlinear regime. For the mass loss rate we deduce $1.4 \times 10^{-7} \mathrm{M}_{\odot} / \mathrm{yr}$ from Fig. 4.6(f).

\subsubsection{Models with enhanced He abundance}

The linear stability analysis for He enhanced abundance $(X=0.20 ; Y=0.78)$ has shown that all models in the mass range from 14 to $40 \mathrm{M}_{\odot}$ are unstable. With respect to the observationally determined masses we have considered the evolution of instabilities for 30 and $34 \mathrm{M}_{\odot}$ models and a low mass model having $17 \mathrm{M}_{\odot}$.

The results for the 30 and $34 \mathrm{M}_{\odot}$ models are almost identical (see Figs. 4.7 and 4.8). Therefore we restrict ourselves to the discussion of the $34 \mathrm{M}_{\odot}$ model. The evolution of radius, velocity, temperature, density and pressure at the surface of the model as well as the variation of the bolometric luminosity as a function of time is shown in Fig. 4.7. Starting from hydrostatic equilibrium and passing the linear phase of exponential growth saturation in the nonlinear regime does not lead to stationary finite amplitude pulsations. Rather the amplitudes of the perturbations vary on a time scale high compared with the pulsation period and the (linear) growth time scale. Finally the amplitudes of the perturbations decrease rapidly and a new static state in hydrostatic equilibrium is established. Thus the instability of the initial model rearranges the structure of the envelope and leads to a new, obviously stable, hydrostatic configuration with increased radius and temperature and decreased density. As a consequence, the absence of finite amplitude pulsations indicates that 55 Cygni cannot be explained on the basis of models with masses above $30 \mathrm{M}_{\odot}$ even with enhanced He abundance.

The evolution of the instability of the $17 \mathrm{M}_{\odot}$ model with enhanced He abundance from hydrostatic equilibrium into the nonlinear regime is shown in Fig. 4.9. The linear domain of exponential growth is dominated by the most unstable mode (see Fig. 4.9.b). Similar to the models with solar chemical composition the envelope is inflated in the nonlinear regime which leads to an increased pulsation timescale (see 4.9.a and b). Associated with the inflation of the envelope is a decrease of surface temperature and density (see Fig. 4.9.c and d). The nonlinear finite amplitude pulsation is irregular and 

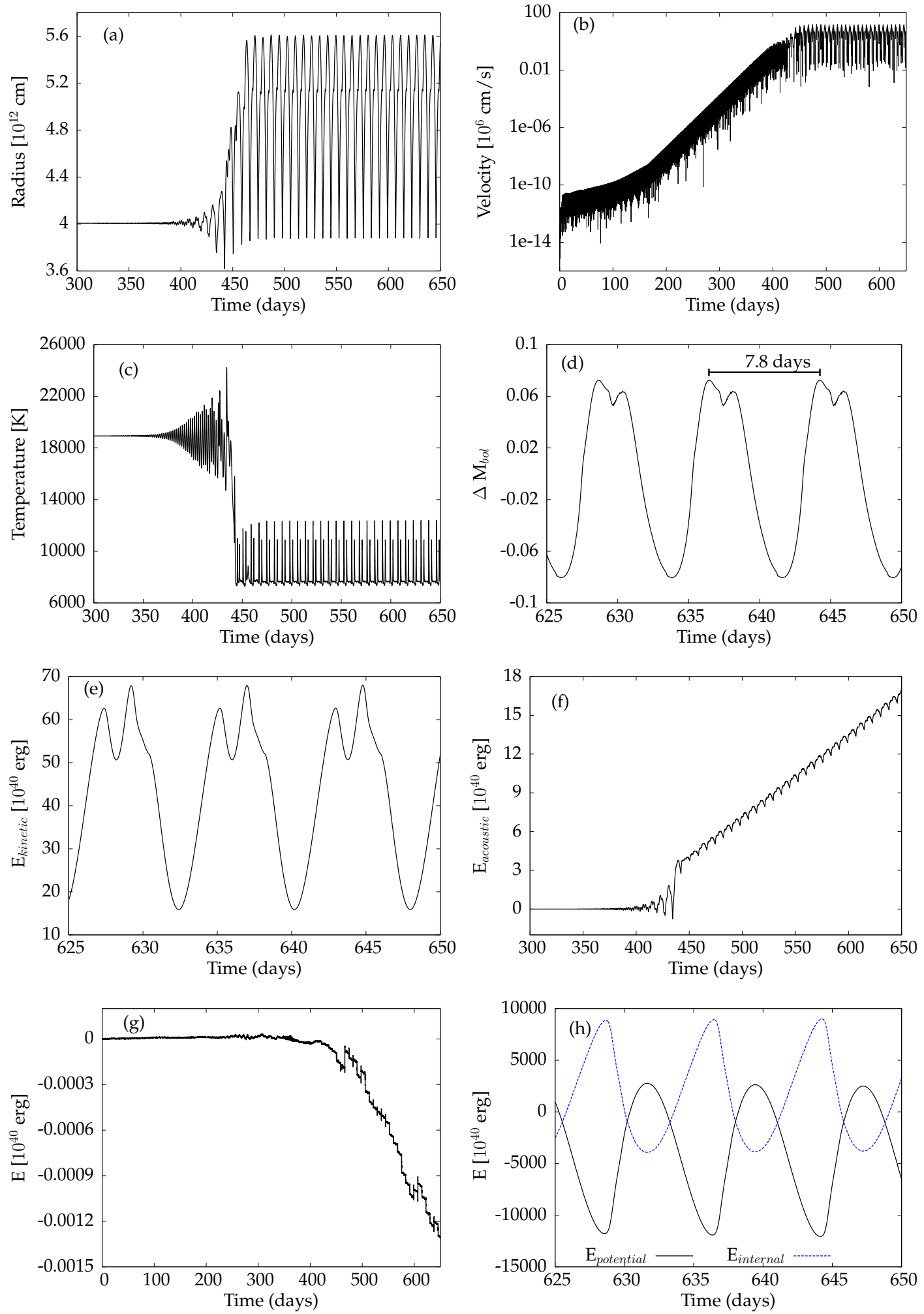

FigURE 4.6: Same as Fig. 4.5 but for a model with $25 \mathrm{M}_{\odot}$. 

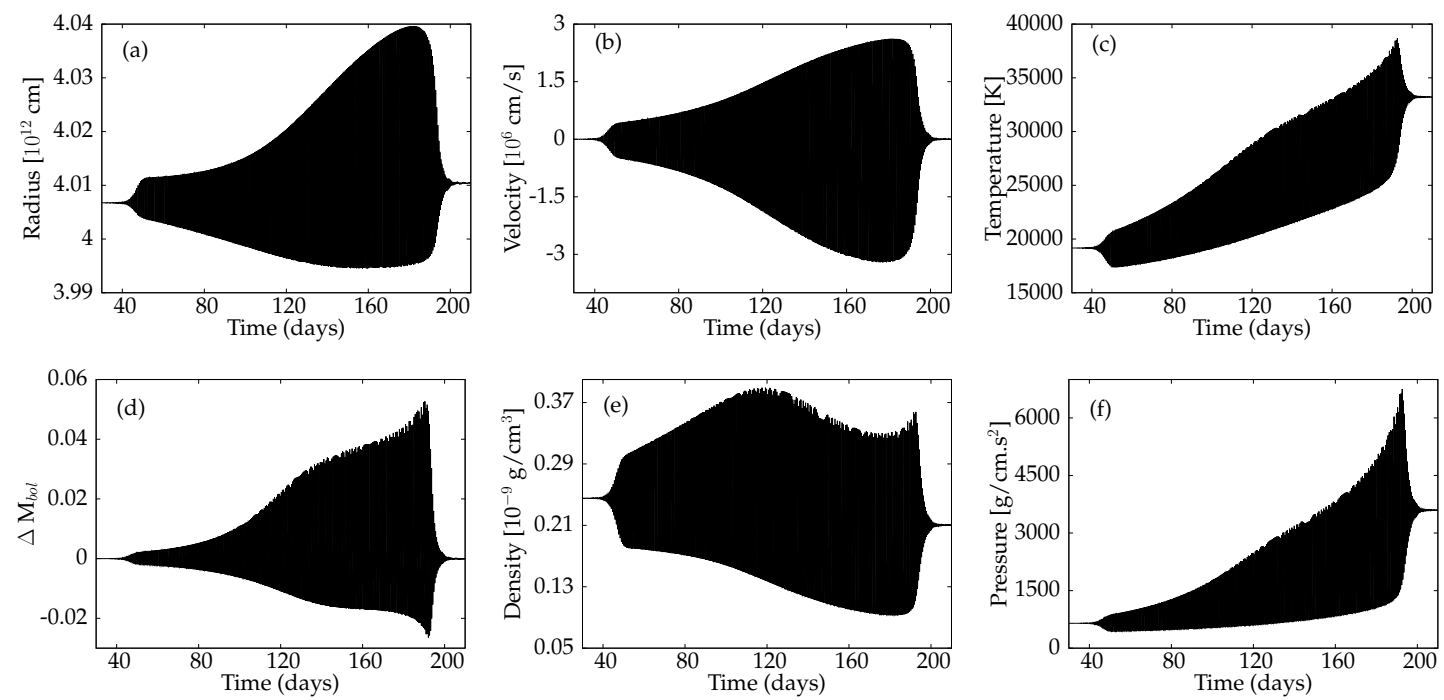

FIGURE 4.7: The evolution of a $34 \mathrm{M}_{\odot}$ model with enhanced He abundance $(X=0.20, Y=0.78)$. The following quantities are given as a function of time: Radius (a), velocity (b) and temperature (c), density (e) and pressure (f) of the outermost grid point, the variation of the bolometric magnitude in $(d)$.
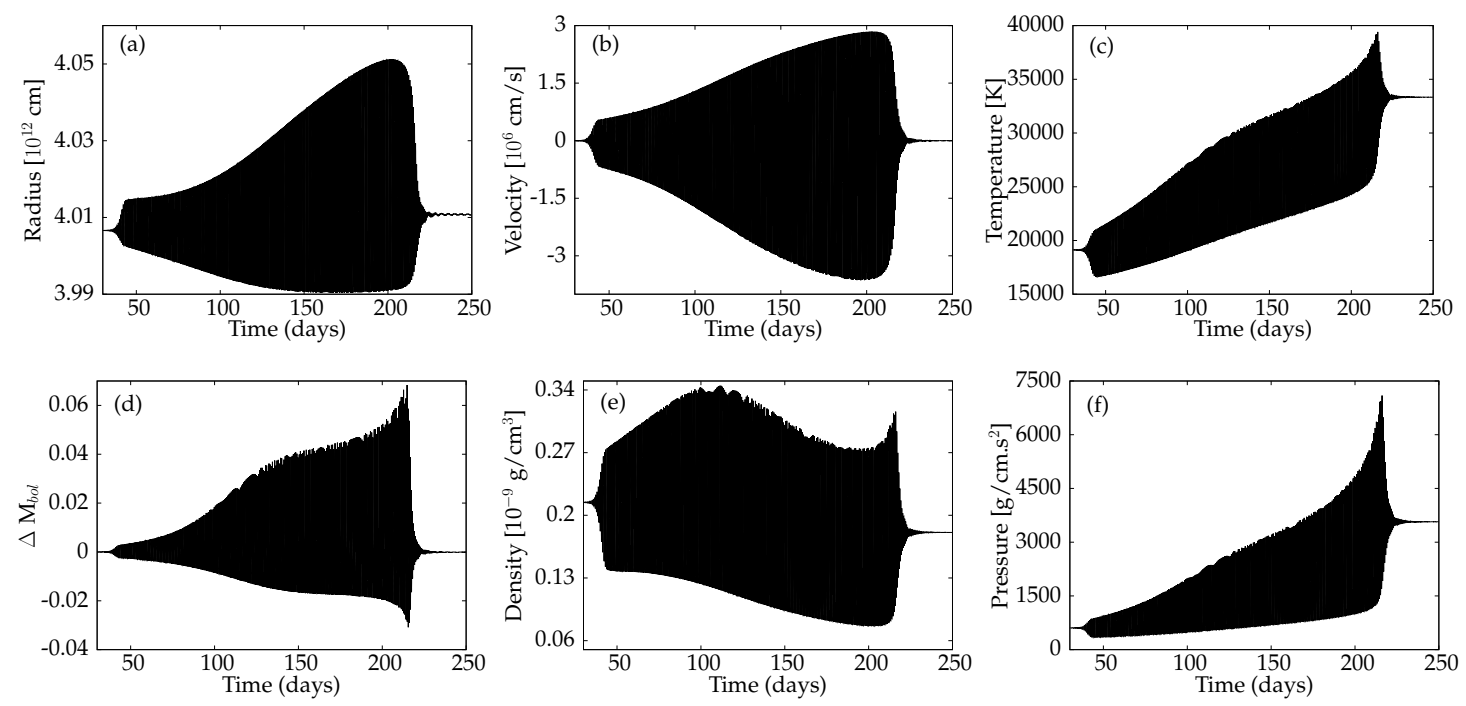

FIGURE 4.8: Same as Fig. 4.7 but for $30 \mathrm{M}_{\odot}$ 

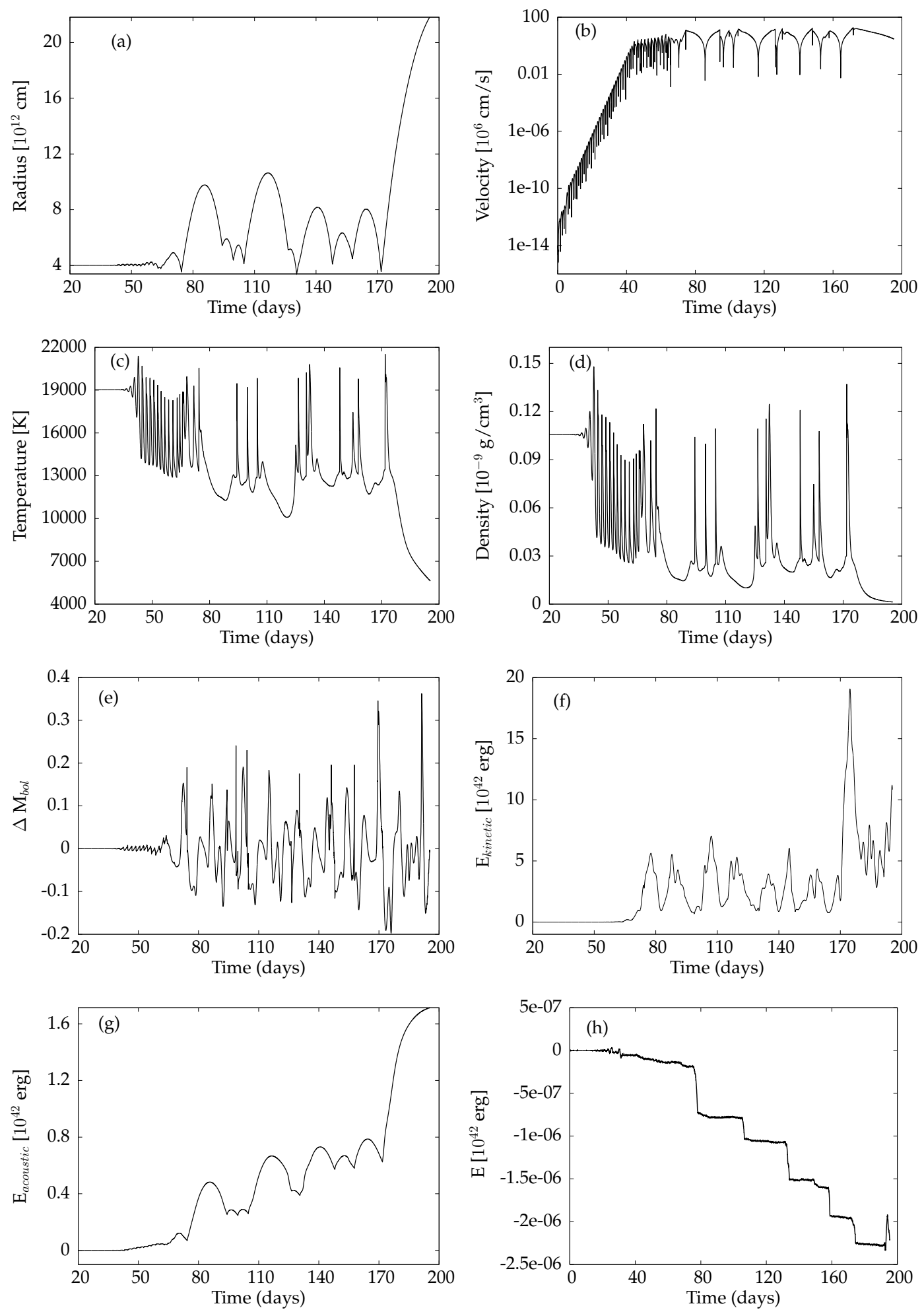

FIGURE 4.9: The evolution of a $17 \mathrm{M}_{\odot}$ model with enhanced He abundance $(X=0.20, Y=0.78)$. The following quantities are given as a function of time: Radius (a), velocity (b), temperature (c) and density (d) at the outermost grid point, the variation of the bolometric magnitude (e), the kinetic energy (f), the time-integrated acoustic luminosity (g) and the error in the energy balance (h). Note that after 170 days the velocity of the outermost grid point exceeds the escape velocity providing direct evidence for mass loss. 
does not exhibit a well defined pulsation period (see Fig. 4.9.c, $\mathrm{d}$ and e). Both the kinetic energy and the time integrated acoustic luminosity (Fig. 4.9.f and g) being smaller than the internal and gravitational energies by two orders of magnitude exceed the error of the energy balance (Fig. 4.9.h) by at least six orders of magnitude. During the final pulsation cycle the surface velocity reaches $159 \mathrm{~km} / \mathrm{s}$ and thus exceeds the escape velocity from the object $(144 \mathrm{~km} / \mathrm{s})$. Due to the associated huge increase in radius and strong decrease in temperature and density (see Fig. 4.9 a, c and d) the simulation had to be stopped because for the range of temperatures and densities reached data for the opacity and the equation of state were no longer available. The fact that the pulsation velocity has exceeded the escape velocity is taken as direct evidence for mass loss. Note that a similar event has been described previously by Glatzel et al. (1999).

\subsection{Discussion and conclusions}

We have performed both a linear stability analysis with respect to radial perturbations and simulations into the nonlinear regime of strange mode instabilities identified in the linear spectrum for sequences of stellar models with solar chemical composition and with enhanced He abundance. Models with enhanced He abundance are linearly unstable over a mass range from 14 to $40 \mathrm{M}_{\odot}$, whereas the maximum mass for instability for solar chemical composition lies around $28 \mathrm{M}_{\odot}$. Nonlinear simulations of models with enhanced He abundance and masses above $30 \mathrm{M}_{\odot}$ indicate that the instability in these cases leads to a new hydrostatic non-variable equilibrium. Should the observed variability of 55 Cygni be due to an instability, this result excludes a high mass for 55 Cygni such as $34 \pm 4 \mathrm{M}_{\odot}$ (Kraus et al., 2015) or $32.1 \mathrm{M}_{\odot}$ (Gies \& Lambert, 1992) and favours low masses such as $23 \mathrm{M}_{\odot}$ (Searle et al., 2008) or even $11 \mathrm{M}_{\odot}$ (Markova \& Puls, 2008).

Nonlinear simulations for models having 23 and $25 \mathrm{M}_{\odot}$ with solar chemical composition and for $17 \mathrm{M}_{\odot}$ with He enhanced abundance exhibit finite amplitude pulsations with a strong indication for mass loss as the final result of the instability. For the 23 and $25 \mathrm{M}_{\odot}$ models the final nonlinear periods of 7.5 and 7.8 days lie within the range of periodicities between 2.7 hours to 22.5 days as derived from observations by Kraus et al. (2015) which is consistent with the periods of 18 and 4.88 days determined by Rufener \& Bartholdi (1982) and Koen \& Eyer (2002) respectively. We emphasize that for the models studied the final nonlinear periods substantially differ from the linear periods since the envelope is inflated in the nonlinear regime of the evolution of the instability. As a consequence, observed periods for these stars should never be compared to linearly determined periods but rather to the period of the final nonlinear finite amplitude pulsation. Some authors (Lefèvre et al., 2009; Percy \& Welch, 1983; van Genderen, 1989) claim that there is no clear evidence for periodic pulsations in 55 Cygni. These findings would be consistent with a very low mass of the object below $17 \mathrm{M}_{\odot}$, where our simulations show an irregular variability rather than strictly periodic pulsations.

From observations of 55 Cygni the following mass loss rates have been determined:

- Kraus et al. (2015) : $2.5 \times 10^{-7} \mathrm{M}_{\odot} / \mathrm{yr}$

- Crowther et al. (2006) : $2.5 \times 10^{-7} \mathrm{M}_{\odot} / \mathrm{yr}$

- Searle et al. (2008) : $5 \times 10^{-7} \mathrm{M}_{\odot} / \mathrm{yr}$

- Markova \& Puls (2008) : (1.17 to 4.07$) \times 10^{-7} \mathrm{M}_{\odot} / \mathrm{yr}$ 
- Barlow \& Cohen (1977) : $5.7 \times 10^{-7} \mathrm{M}_{\odot} / \mathrm{yr}$

These mass loss rates may be compared with the mass loss rates estimated on the basis of our simulations for the 23 and $25 \mathrm{M}_{\odot}$ models with solar abundances and obtained as $2.6 \times 10^{-7} \mathrm{M}_{\odot} / \mathrm{yr}$ and $1.4 \times 10^{-7} \mathrm{M}_{\odot} / \mathrm{yr}$, respectively. The coincidence with the observed values is remarkable. For comparison, mass loss rates for the 23 and $25 \mathrm{M}_{\odot}$ models based on the wind-momentum luminosity relation (see, e.g., Lamers \& Cassinelli, 1999) amount to $1.9 \times 10^{-6} \mathrm{M}_{\odot} / \mathrm{yr}$ and $1.8 \times 10^{-6} \mathrm{M}_{\odot} / \mathrm{yr}$, respectively, being higher than the acoustically driven mass loss rates by one order of magnitude. Although a mass loss rate could not be determined, we found direct evidence for mass loss, if the mass is reduced to $17 \mathrm{M}_{\odot}$. In this model the velocity of the surface of the model has exceeded the escape velocity. In general an increase of the mass loss rate with decreasing mass (at constant luminosity) is expected, since the strange mode instability becomes stronger with increasing luminosity to mass ratio.

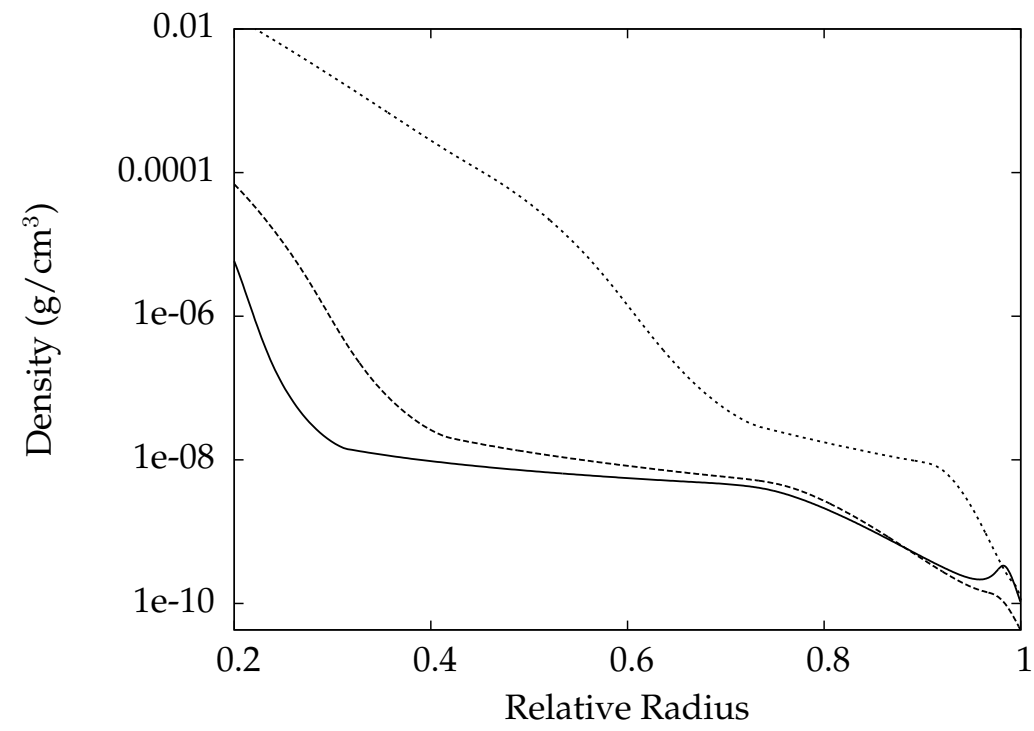

FIGURE 4.10: Density as a function of relative radius for the $25 \mathrm{M}_{\odot}$ model (dashed line) and the $17 \mathrm{M}_{\odot}$ model (full line) of 55 Cygni and for a model of the LBV AG Car (dotted line).

The envelope structure in terms of the density stratification for two of our models (with $25 \mathrm{M}_{\odot}$ and $17 \mathrm{M}_{\odot}$ ) of 55 Cygni is compared with that of a model for a luminous blue variable (LBV) in Fig. 4.10. For the LBV model we have chosen parameters close that of AG Car $\left(\log L / L_{\odot}=5.9, \log \mathrm{T}_{\text {eff }}=4.4, \mathrm{M}=60 \mathrm{M}_{\odot}\right.$, solar chemical composition, see also Fig. 2 in Kiriakidis et al., 1993). All three models suffer from strange mode instabilities, but only the $17 \mathrm{M}_{\odot}$ model exhibits a density inversion, which shows that the density inversion is not the primary origin of strange mode instabilities. Rather dominant radiation pressure is responsible for it, as demonstrated by Glatzel (1994). However, if the fraction of radiation pressure increases, simultaneously with the increase of the growth rate of strange mode instabilities, density inversions are likely, but do not necessarily occur. Vice versa, density inversions are found even when the fraction of radiation pressure is small. For a detailed discussion of the origin of density inversions we refer to Glatzel \& Kiriakidis (1993b). The high fraction of radiation pressure both in the models for 55 Cygni and in models for LBVs suggests that similar 
results as those found in this paper for 55 Cygni models (finite amplitude pulsations, envelope inflation, mass loss) are also expected for models of LBVs.

The present study has been restricted to spherically symmetric hydrostatic stellar models and to radial perturbations of them. With respect to a linear stability analysis we also expect nonradial modes to be unstable. As shown in the present paper for the stellar models studied the predictions of the linear approximation (e.g., concerning the pulsation periods) substantially differ from nonlinear behaviour. Thus a nonlinear study is inevitable. However, so far a nonlinear treatment of nonradial modes is not available. Therefore the consideration of nonradial modes has been entirely disregarded in the present study. A linear nonradial stability analysis of models for 55 Cygni will be presented elsewhere. A final cautionary remark concerns the influence of rotation and magnetic fields on the stability, pulsations and mass loss of models for 55 Cygni. Studies in this direction taking nonlinear effects fully into account are not yet feasible. Therefore the present study is meant as a first preliminary step towards an understanding of the variability and mass loss of 55 Cygni. 


\section{Chapter 5}

\section{Monotonically unstable modes in main sequence stars}

\subsection{Introduction}

In general, the eigenfrequencies determined by a linear non adiabatic stability analysis of stellar models have non vanishing real and imaginary parts. A special kind of eigenfrequencies with zero real parts and negative imaginary parts have been identified by Deller (2009); Hilker (2009) and Saio (2011). Saio (2011) found that this type of monotonically unstable radial modes appears in models for massive stars with solar chemical composition having masses above $60 \mathrm{M}_{\odot}$. Their relative maximum of the kinetic energy derived from their eigenfunctions is found at the bottom of the convection zone associated with the Fe-opacity bump. Although the origin of this type of modes is not completely understood, Saio (2011) suggested their origin to be due to the radiative luminosity approaching the Eddington luminosity in the stellar envelope. He also emphasizes that the appearance of the monotonically unstable mode roughly coincides with the Humphreys Davidson limit (Humphreys \& Davidson, 1979) and suggests that it may induce optically thick winds in massive stars.

In this study, we shall systematically investigate the behaviour and characteristics of monotonically unstable modes in zero age main sequence (ZAMS) models. This will be done both considering radial and nonradial perturbations for the linear stability analysis. Whether monotonically unstable modes can also be identified in the nonradial spectrum, will be of particular interest. A study of monotonically unstable modes is motivated by the fact that their growth rates may be higher than those of low order p-modes or strange modes (see Saio, 2011).

The construction of stellar models is discussed in section 5.2, the stability analysis is described in section 5.3, and the results are presented in section 5.4. Our conclusions follow (section 5.5).

\subsection{Stellar Models}

The MESA code, version: 6208, (Paxton et al., 2011, 2013, 2015) has been used to determine luminosities and effective temperatures as a function of mass along the ZAMS, where the ZAMS is defined as the stage when one per cent of the hydrogen has been burnt in the center. Zero age main sequences have been calculated for the following three chemical compositions:

- $\mathrm{Z}=0.03, \mathrm{Y}=0.28$ and $\mathrm{X}=0.69$

- $\mathrm{Z}=0.02, \mathrm{Y}=0.28$ and $\mathrm{X}=0.70$ 


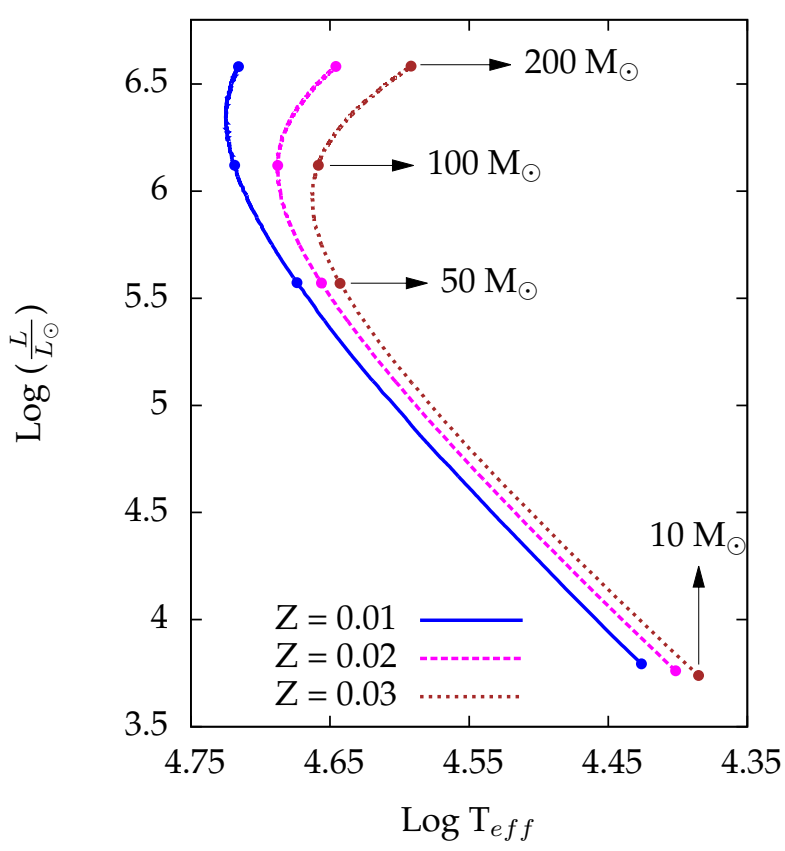

FIGURE 5.1: Zero age main sequences in the HRD for three different metallicities.

- $\mathrm{Z}=0.01, \mathrm{Y}=0.28$ and $\mathrm{X}=0.71$

They are shown in an HRD in Fig. 5.1. On the basis of these results, ZAMS envelope models have been constructed in the mass range between 10 and $160 \mathrm{M}_{\odot}$. In these calculations, nuclear reactions and nuclear energy generation is disregarded, convection is treated according to standard mixing length theory (Böhm-Vitense, 1958) with a mixing length of 1.5 pressure scale heights, OPAL tables have been used for the opacity, and the equations of stellar structure have been integrated up to some conveniently chosen cutoff temperature. Disregarding nuclear reactions implies that their influence on stellar stability, i.e., that the $\varepsilon$-mechanism is excluded in our study. This approach is justified as previous studies (see, e.g., Glatzel \& Kiriakidis, 1993a) prove that the $\varepsilon$-mechanism does not severely affect the stability of stars. It is restricted to the fundamental mode only with growth rates of the order of the nuclear (stellar evolution) timescale. Moreover, $\varepsilon$-instability occurs only close to the ZAMS. Thus $\varepsilon$-instability competes with stellar evolution and perturbations will grow at most by one order of magnitude before the star has left the domain of $\varepsilon$-instability. Therefore the $\varepsilon$-mechanism is to be regarded to be only of academic interest.

According to standard textbooks on stellar structure and evolution, massive main sequence stars have convective cores and radiative envelopes (Iben, 2013; Kippenhahn et al., 2012; Salaris \& Cassisi, 2006). However, in the envelopes of massive stars small convection zones may appear (Glatzel et al., 1993; Stothers \& Chin, 1993) which are due to opacity maxima (Rogers \& Iglesias, 1992). Fig. 5.2 illustrates the presence of convection zones in the envelopes of selected ZAMS models. In the $10 \mathrm{M}_{\odot}$ model two convection zones are present while for 50 and $100 \mathrm{M}_{\odot}$ only one convection zone can be identified. Possible consequences of these convection zones in hot massive stars are discussed in detail by Cantiello et al. (2009). 

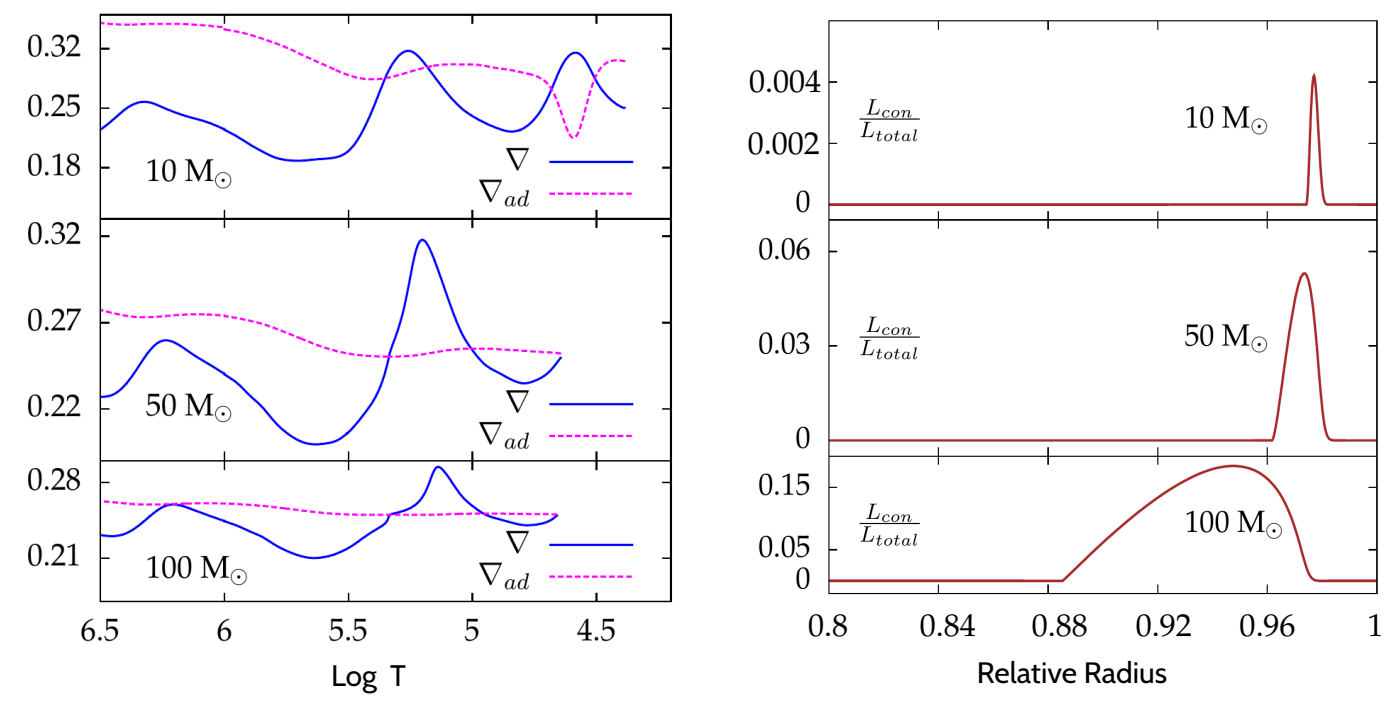

FIGURE 5.2: Convection zones $\left(\nabla>\nabla_{a d}\right)$ of three ZAMS models with metallicity $\mathrm{Z}=0.03$ in terms of $\nabla$ and $\nabla_{a d}$ as a function of temperature (left panel). In the right panel, the corresponding ratios of the convective luminosity to the total luminosity are given as a function of relative radius.

\subsection{Stability Analysis}

For the models discussed in the previous section, we have performed a radial as well as a non-radial non-adiabatic linear stability analysis. The set of pulsation equations is taken in the form described by Glatzel \& Gautschy (1992). With six boundary conditions (three on the top and three at the bottom of the envelopes) these pulsation equations form a boundary eigenvalue problem. It is solved using the Ricatti method (Gautschy \& Glatzel, 1990a) as a nonlinear initial value problem with prescribed accuracy. The solution of this eigenvalue problem provides an infinite set of complex eigenfrequencies and corresponding eigenfunctions. The real part of the eigenfrequency $\left(\sigma_{r}\right)$ corresponds to the pulsation period while the imaginary part $\left(\sigma_{i}\right)$ provides information on the stability. $\sigma_{i}>0$ corresponds to damping and $\sigma_{i}<0$ indicates excitation and instability. The eigenfrequencies discussed below are normalized by the global free fall time $\tau_{\mathrm{ff}}\left(\right.$ with $\left.\tau_{\mathrm{ff}}^{-1}=\sqrt{\frac{3 G M}{R^{3}}}\right)$.

Convection and convection zones may have an important influence on stellar stability and pulsations (Dupret et al., 2005; Grigahcène et al., 2005). However, a theory for the interaction of convection and pulsation is still missing. In our investigation, it is described by adopting the 'frozen-in-approximation' (Baker \& Kippenhahn, 1965). It consists of neglecting the Lagrangian perturbation of the convective flux and holds, if the convection timescale is much longer than the pulsation timescale. It is usually applied, if the fraction of energy transported by convection is small compared to the total energy flux. Fig 5.2 shows the contribution of the convective luminosity to the total luminosity for three representative ZAMS models. For the models with 10, 50 and $100 \mathrm{M}_{\odot}$ the fraction of convective luminosity amounts to $<0.5 \%,<6 \%$ and $<18 \%$ of the total luminosity, respectively. This indicates that the convective luminosity does not play a significant role here and that the frozen-in-approximation may be assumed to hold. 

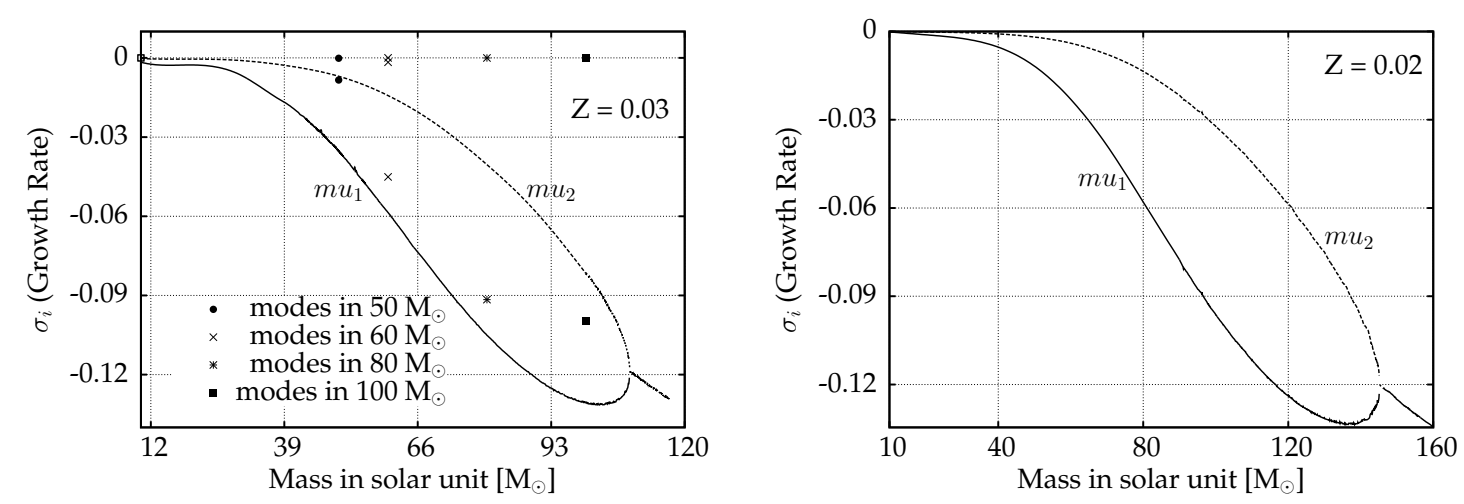

FIGURE 5.3: Growth rates (normalized by the global free fall time) of unstable nonradial modes with $l=2$ as a function of mass for ZAMS models with metallicity $Z=0.03$ (left panel) and $Z=0.02$ (right panel). $\mathrm{mu}_{1}$ and $\mathrm{mu}_{2}$ denote two for low masses monotonically unstable modes which merge to form an oscillatory unstable mode for high masses.

\subsection{Results}

\subsubsection{Radial and non-radial monotonically unstable modes}

Monotonically unstable modes have been identified in ZAMS models both for radial and non-radial perturbations. With respect to radial perturbations we found only one mode to be present in very massive $\left(>147 \mathrm{M}_{\odot}\right.$ for $\left.\mathrm{Z}=0.03\right)$ stellar models. For $l=$ 2 , at least two monotonically unstable modes have been identified by our non-radial analysis: Two highly unstable monotonic modes appear as a pair in the modal diagram shown in Fig. 5.3 both for high metallicity $(Z=0.03$, left panel) and solar metallicity $(Z$ $=0.02$, right panel). For comparison, growth rates of representative unstable low order acoustic modes are added in Fig. 5.3. The monotonically unstable modes merge (at $\mathrm{M}=109 \mathrm{M}_{\odot}$ for $\mathrm{Z}=0.03$ and at $\mathrm{M}=147 \mathrm{M}_{\odot}$ for $\mathrm{Z}=0.02$ ) thus forming an oscillatory unstable mode for higher masses. The merging point shifts towards higher masses with decreasing metallicity (see Fig. 5.3).

\subsubsection{Dependence on harmonic degree $(l)$ of monotonically unstable modes}

The dependence on harmonic degree of the monotonically unstable modes has been investigated systematically. The results are shown in Fig. 5.4, where the real and imaginary parts of the eigenfrequencies are given as a function of the harmonic degree for three ZAMS models with metallicity $\mathrm{Z}=0.03$. The growth rates increase with the harmonic degree up to a maximum value and decrease for even higher $l$. According to Fig. 5.4, the monotonically unstable modes pair at a mass dependent value of the harmonic degree $\left(\right.$ at $l \approx 450$ for $10 \mathrm{M}_{\odot}$, at $l \approx 170$ for $50 \mathrm{M}_{\odot}$ and at $l \approx 3$ for $\left.100 \mathrm{M}_{\odot}\right)$ thus forming an oscillatory strongly unstable mode for very high values of $l$.

\subsubsection{Distribution of kinetic energies of monotonically unstable modes}

The distribution of the kinetic energy of modes, in particular the monotonically unstable modes, can be derived from the corresponding eigenfunctions. It is shown in Figs. 5.5 and 5.6 for three ZAMS models with metallicity $Z=0.03$. As the work integral is not defined for non oscillatory modes, the distribution of the kinetic energy might provide 

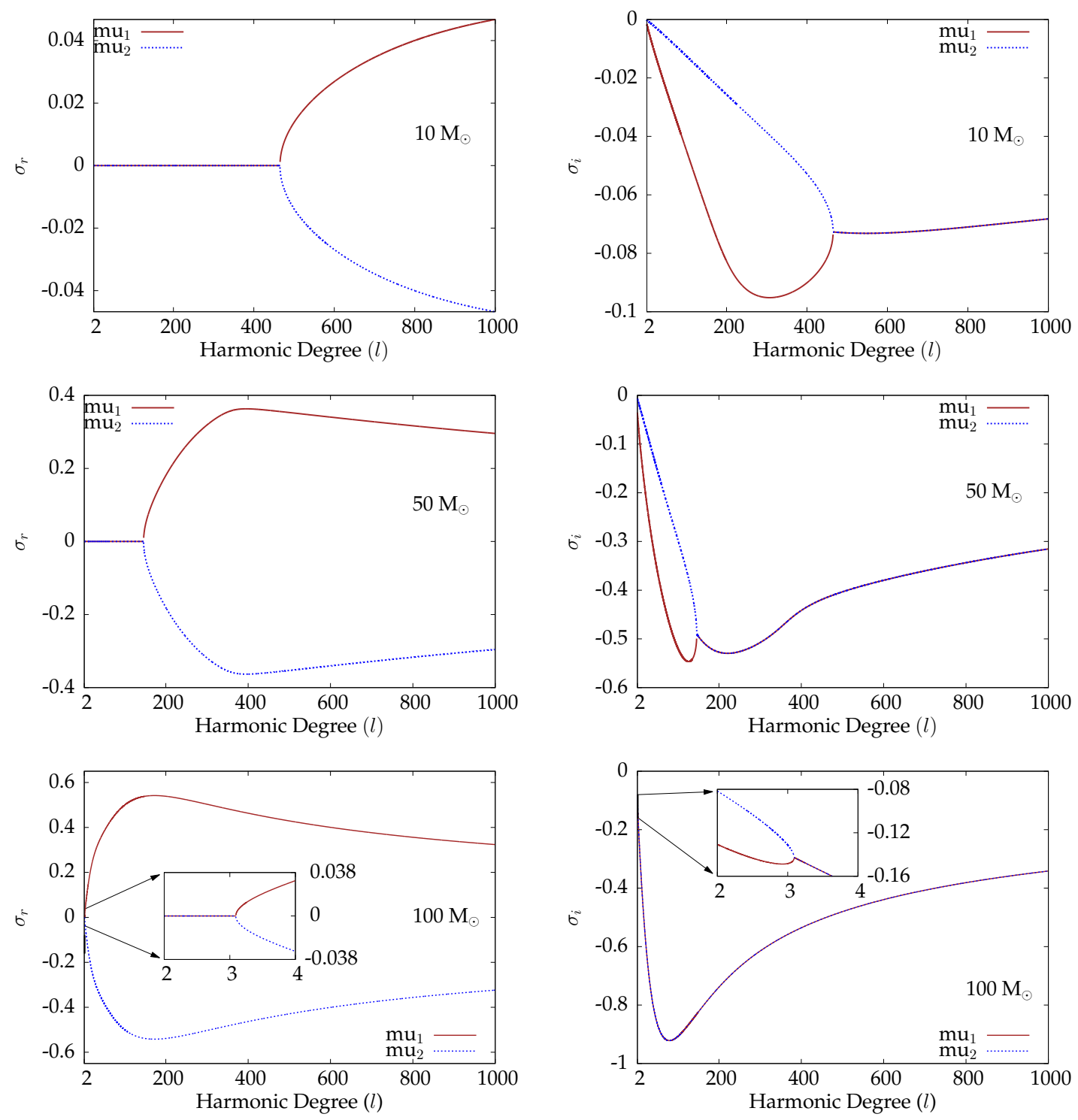

FIGURE 5.4: Real and imaginary parts of the eigenfrequencies (normalized by the global free fall time) of unstable modes associated with the monotonically unstable modes $\mathrm{mu}_{1}$ and $\mathrm{mu}_{2}$ as a function of harmonic degree $(l)$ for three ZAMS models with metallicity $Z=0.03$. Note the mode pairing of the monotonically unstable modes at a certain mass dependent value of the harmonic degree providing an unstable oscillatory mode for high $l$. 

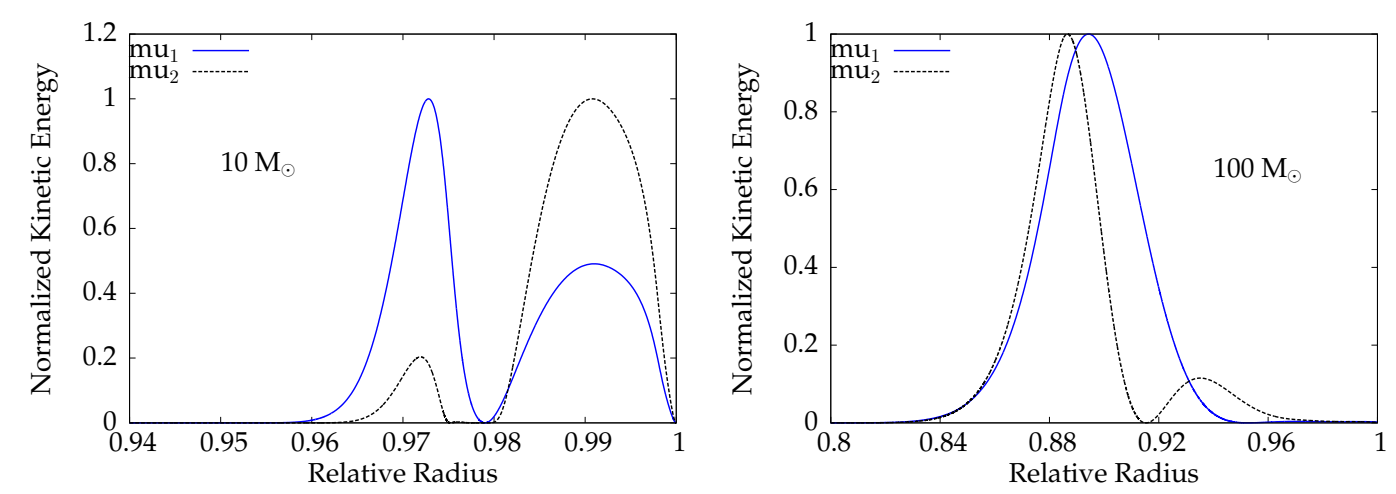

FIGURE 5.5: Normalized kinetic energy as a function of relative radius of the two monotonically unstable modes $\mathrm{mu}_{1}$ and $\mathrm{mu}_{2}$ with $l=2$ for two ZAMS models having the metallicity $\mathrm{Z}=0.03$.

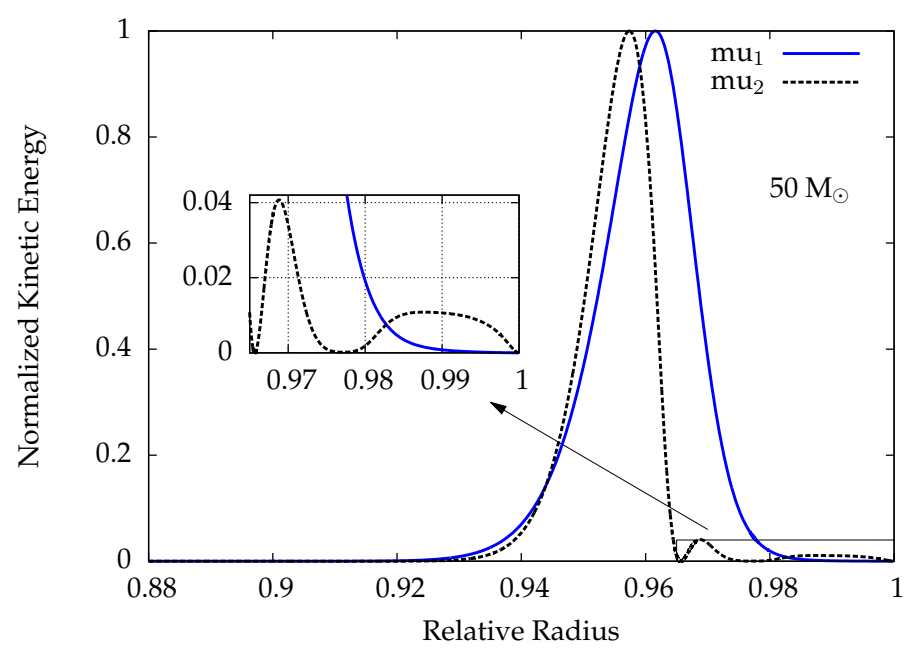

FIGURE 5.6: Same as Fig. 5.5 but for a ZAMS model with $50 \mathrm{M}_{\odot}$.

some information on the excitation of the monotonically unstable modes and on their domain of existence. Figs. 5.5 and 5.6 show that a maximum of the kinetic energy is found at the bottom of the convection zone associated with the Fe-opacity bump. For the low mass model with $10 \mathrm{M}_{\odot}$, a second maximum appears between the Fe-opacity convection zone and He II convection zone. This is for the mode $\mathrm{mu}_{2}$, contrary to mode $\mathrm{mu}_{1}$, the position of the absolute maximum of the kinetic energy. Secondary, less pronounced maxima may appear close to the photosphere (see Figs. 5.5 and 5.6).

\subsection{Discussion and conclusions}

The monotonically unstable modes identified in this study are present both for radial as well as non-radial perturbations. Hence their existence is independent of the geometry of the perturbations. The present analysis is based on ZAMS models but we suspect that the monotonically unstable modes are also present in later stages of stellar evolution. Their growth rates are higher for non-radial perturbations compared to radial perturbations and their presence in models with $10 \mathrm{M}_{\odot}$ suggests that they can affect $\beta$ - Cepheid type stars close to the main sequence. The distribution of the kinetic 


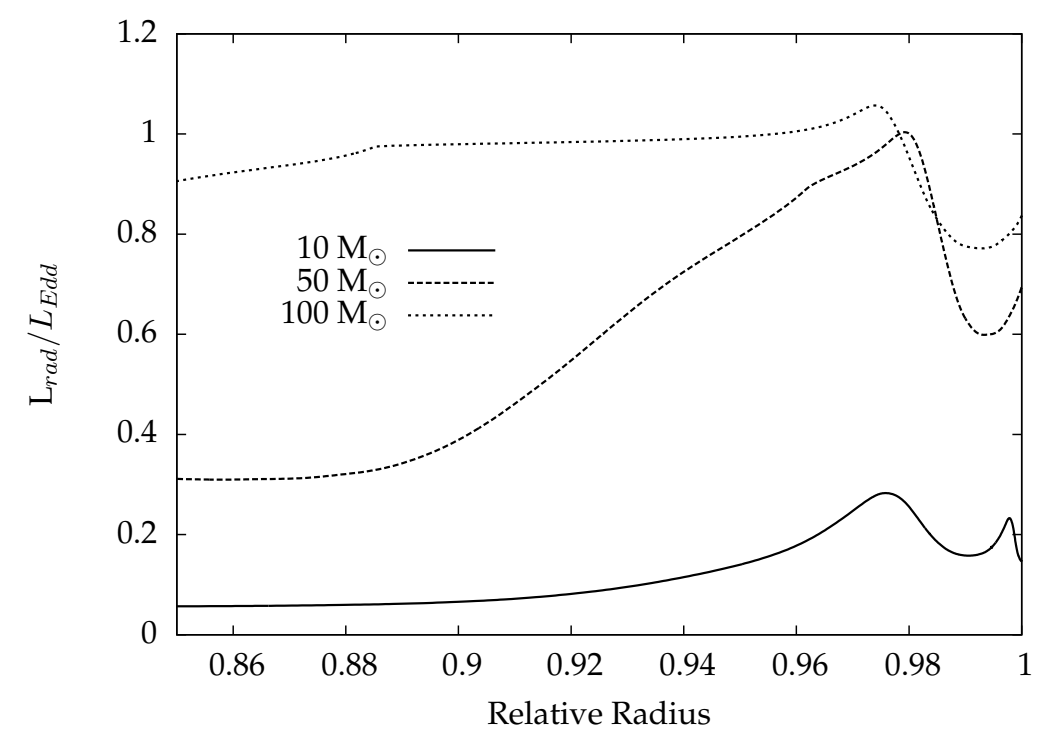

FIGURE 5.7: Ratio of the radiative and the Eddington luminosity as a function of relative radius for three ZAMS models with metallicity $\mathrm{Z}=$ 0.03 .

energy for these modes exhibits a local maximum at the bottom of the Fe-opacity convection zone. A second local maximum of the kinetic energy is found for $10 \mathrm{M}_{\odot}$ models close to the stellar surface. Our findings concerning the kinetic energy of the monotonically unstable modes for the massive ZAMS models are similar to those of Saio (2011). However, in the models studied by Saio (2011), the bottom of the Fe-opacity convection zone is situated deeper within the stellar envelope and the models studied by him have a more pronounced core envelope structure.

The dependence on the harmonic degree $l$ of the monotonically unstable modes has been studied by following them up to $l=1000$ where an interesting phenomenon has been discovered. When increasing $l$, two monotonically unstable modes merge at a critical value of $l\left(l \approx 480\right.$ for $10 \mathrm{M}_{\odot}, l \approx 150$ for $50 \mathrm{M}_{\odot}$ and $l \approx 3$ for $100 \mathrm{M}_{\odot}$, see Fig. 5.4) and form an unstable oscillatory mode with a frequency in the g-mode range $\left(\sigma_{r}<1\right)$.

Saio (2011) suggested that the origin of his radial monotonically unstable modes is due to the radiative luminosity being close to the Eddington luminosity $\left(\mathrm{L}_{r a d} / \mathrm{L}_{E d d} \approx\right.$ 1 ) in a large fraction of the stellar envelope. The latter is also true for radial monotonically unstable modes in our main sequence models. However, $\mathrm{L}_{r a d} / \mathrm{L}_{E d d} \approx 1$ is not a necessary condition for the occurrence of non-radial monotonically unstable modes: Fig. 5.7 shows the ratio of the radiative to the Eddington luminosity $\left(\mathrm{L}_{\text {rad }} / \mathrm{L}_{\text {Edd }}\right)$ as a function of the normalized radius in the envelopes of ZAMS models with 10, 50 and $100 \mathrm{M}_{\odot}$. Although for the entire envelope of the $10 \mathrm{M}_{\odot}$ model we have $\mathrm{L}_{r a d} / \mathrm{L}_{E d d}<$ 0.3 nonradial monotonically unstable modes can be identified here. Thus the nonradial monotonically unstable modes cannot be related to the existence of the HD limit (Humphreys \& Davidson, 1979). In this respect we note that Saio (2011) claims that the occurrence of the radial monotonically unstable modes studied by him coincides with the HD limit and leads to optically thick winds. We emphasize that this conclusion is premature, since the final result of an instability can only be determined by nonlinear calculations and never by a linear analysis. 



\section{Chapter 6}

\section{Summary and future work}

\subsection{Summary}

Linear stability analyses have been performed for models of massive main sequence stars and models of the B-type supergiant 55 Cygni (HD 198478). The instabilities identified have been followed into the nonlinear regime with the help of a time implicit fully conservative scheme. The final velocity amplitudes are in any case comparable with the escape velocity of the corresponding models. For selected models pulsation velocities exceed the escape velocity which is taken as direct evidence for mass loss. The nonlinear simulations provide an estimate for the mass loss rate which appears to be consistent with observations. Saio (2011) reported on the presence of monotonically unstable modes in models of massive stars. The properties of these modes have been investigated systematically for main sequence models and their existence even in the nonradial spectrum has been established. The three main issues of this thesis may be summarized in more detail as follows:

\subsubsection{Main sequence stars}

Since pulsations have been observed in many massive stars, we have performed a systematic study of radial pulsations and instabilities in models for massive main sequence stars with solar chemical composition both in the linear as well as in the nonlinear regime. The investigation is based on a sequence of envelope models for upper main sequence stars in the mass range between 50 to $150 \mathrm{M}_{\odot}$. These models are tested for linear stability. The linear stability analysis shows that models having masses above $58 \mathrm{M}_{\odot}$ are unstable. To determine the final fate of the unstable envelopes we have performed nonlinear simulations for selected models. These nonlinear simulations show that the instabilities lead to periodic pulsations with periods between 3 hours and 1 day for the models considered. The final finite amplitude pulsations generate an acoustic flux which can cause a pulsationally driven stellar wind. On the basis of our simulations we were able to estimate the associated mass loss rate which was found to lie in the range of $0.25-0.7 \times 10^{-7} \mathrm{M}_{\odot} / \mathrm{yr}$.

\subsubsection{Cygni (HD 198478)}

55 Cygni is a variable supergiant. Recent observational studies revealed that this star pulsates in pressure, gravity and strange modes. The pulsations seem to be associated with episodes of mass loss. In this thesis we have presented a theoretical study of stellar models with parameters close to that of 55 Cygni. A linear nonadiabatic stability analysis with respect to radial perturbations was performed and the evolution of instabilities into the nonlinear regime was followed by numerical simulation. Our study indicates that the mass of 55 Cygni lies below $28 \mathrm{M}_{\odot}$. As the final consequence of the 
instabilities the nonlinear simulations revealed finite amplitude pulsations with periods consistent with the observations. The nonlinear results also indicate a connection between pulsations and mass loss and allow for an estimate of the mean mass loss rate. It is consistent with the observed values.

\subsubsection{Monotonically unstable modes}

We have performed an extensive linear stability analysis of models for main sequence stars with masses in the range between 10 and $160 \mathrm{M}_{\odot}$. A set of non oscillatory, monotonically unstable modes (with purely imaginary eigenfrequencies) has been identified both for radial and nonradial perturbations. Some of these modes grow on the dynamical timescale and therefore may severely affect the stellar structure. The growth rate sensitively depends on the mass of the model and on the harmonic degree $(l)$ of the perturbation. Maximum growth rates are found between $l \approx 1$ and 300 depending on the mass of the model. At high masses two monotonically unstable modes may pair to form an oscillatory unstable mode. Considering the eigenfunctions of these modes their kinetic energy in general exhibits a maximum value at the bottom of the convection zone associated with the Fe-opacity bump. A secondary maximum appears close to the stellar surface in some models. Whether these modes and their growth rates are affected by the treatment of convection still needs to be investigated.

\subsection{Future work}

Observations using space and ground telescopes are crucial to test theoretical predictions for the pulsations of stars. Recent observational studies (see, e.g., Kraus et al., 2015) suggest that the supergiant 55 Cygni pulsates in radial as well as in nonradial modes. As a part of this thesis, radial perturbations have been considered and a radial linear stability analysis has been performed together with nonlinear simulations of the evolution of instabilities for a set of models for 55 Cygni. A nonradial linear stability analysis is still missing and needs to be done in order to understand the overall variability of 55 Cygni. This will enable us to compare the observed periods with the periods predicted by both the radial and nonradial stability analysis at least in the linear regime. Unfortunately, nonlinear nonradial simulations are not feasible since appropriate numerical tools are not yet available. Accordingly we intend to perform a nonradial linear stability analysis for models of 55 Cygni in the near future.

The B-type supergiant HD 50064 was monitored by CoRoT (a French-European space telescope) as well as by ground based telescopes. These observations indicate that the star HD 50064 pulsates with a period of 37 days and the pulsation is clearly found to be connected with its variable mass loss (Aerts et al., 2010b). Aerts et al. (2010b) have identified the dominant period found in HD 50064 with a strange mode. These findings are consistent with the general trend that stellar models with high luminosity to mass ratios ( $>10^{3}$ in solar units) exhibit strange mode instabilities. Moreover, the instabilities associated with these strange modes tend to lead to mass loss, as also confirmed in this thesis. Due to its high luminosity to mass ratio, the observed star HD 50064 is a very good candidate for strange mode instabilities and to study the connection between pulsation and mass loss. In fact, preliminary nonlinear simulations show that the final surface velocity amplitude can easily attain a value close to $(\approx 30 \%)$ the escape velocity for models of HD 50064. As an example, the results of the simulation for a model having $\mathrm{T}_{\text {eff }}=13500 \mathrm{~K}, \log \left(\mathrm{L} / \mathrm{L}_{\odot}\right)=6.1$ and a mass of $55 \mathrm{M}_{\odot}$ are displayed 

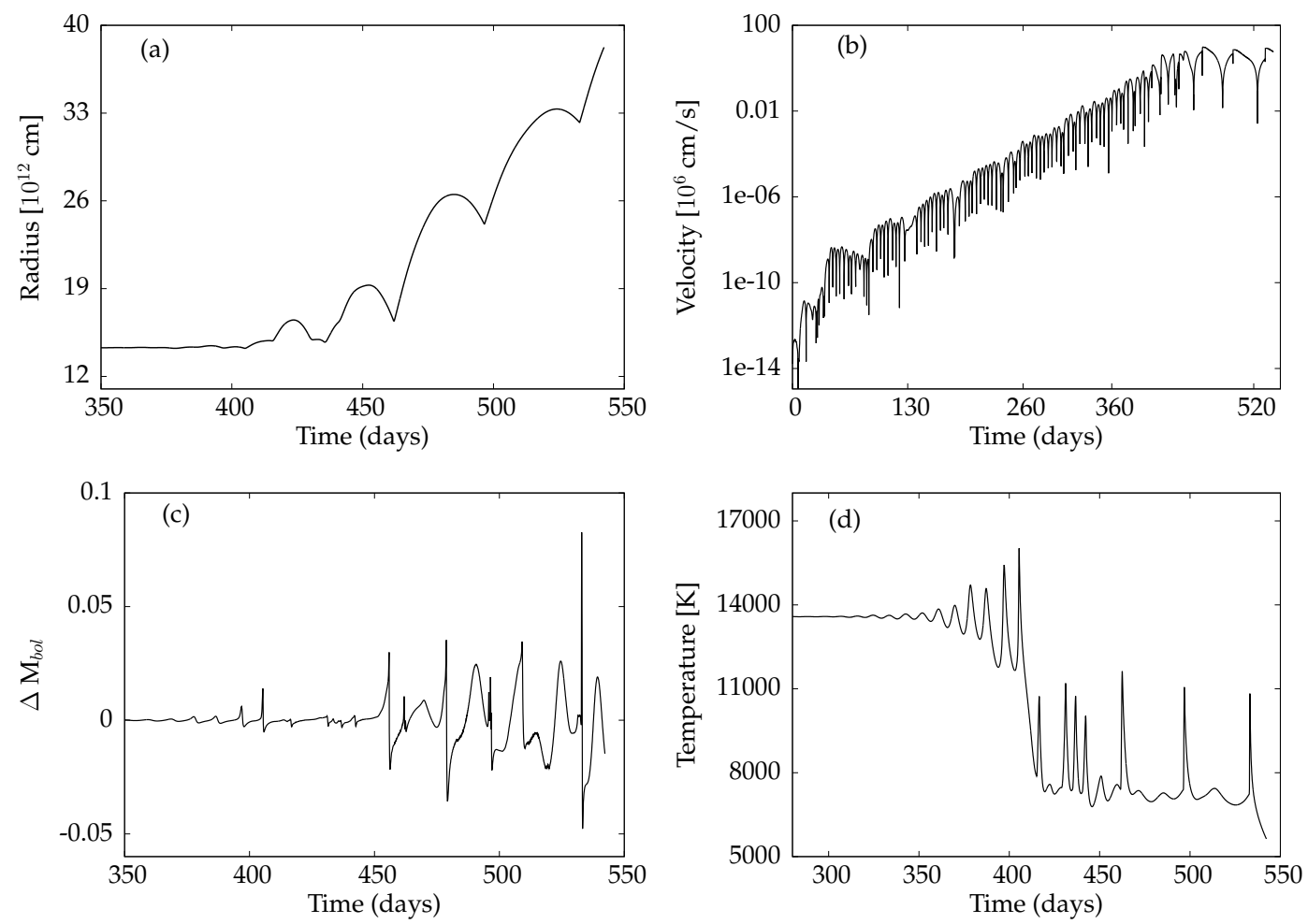

FIGURE 6.1: Nonlinear evolution of the instability of a chemically peculiar (enhanced helium) model for HD 50064 having $\mathrm{T}_{\text {eff }}=13500 \mathrm{~K}, \log$ $\left(\mathrm{L} / \mathrm{L}_{\odot}\right)=6.1$ and $\mathrm{M}=55 \mathrm{M}_{\odot}$. The radius (a), the surface velocity $(\mathrm{b})$, the surface temperature (d) and the variation of the bolometric luminosity

(c) is given as a function of time.

in Fig. 6.1, where the variation of radius, surface velocity, bolometric luminosity and surface temperature is given as a function of time. Note that in the nonlinear regime the pulsation period increases due to an increased mean radius of the model. The intended thorough stability analysis followed by nonlinear simulations for models of HD 50064 is expected to provide further insights into the variability and mass loss of this star.

Apart from B-type supergiants, photometric and spectral variabilities have also been discovered in O-type supergiants (see, e.g., Howarth \& Stevens, 2014; Markova et al., 2005; Prinja et al., 2006). The origin of these variabilities is not yet fully understood. Therefore a linear stability analysis followed by nonlinear simulations for models of O-type supergiants is expected to enhance our understanding of pulsation related variabilities in these stars and is intended for near future. Similar to the B-type supergiants, nonlinear simulations will provide the acoustic fluxes associated with the final finite amplitude pulsations and thus an estimate for the pulsationally driven mass loss. Preliminary results of a stability analysis and nonlinear simulations of instabilities for models of $\zeta$ Puppis (HD 66811) - an O-type supergiant - are displayed in Fig. 6.2 and Fig. 6.3 respectively. The stability analysis for models having enhanced He abundances ( $\mathrm{Y}=0.58, \mathrm{Z}=0.02$, consistent with the observations) shows that models less massive than $97 \mathrm{M}_{\odot}$ are unstable with respect to radial perturbations. Following the instabilities into the nonlinear regime at least one pulsation period of 1.4 days can be identified in the variation of bolometric luminosity for a model having $44 \mathrm{M}_{\odot}$. It is close to the observational period of 1.78 days suggested by Howarth \& Stevens (2014) 

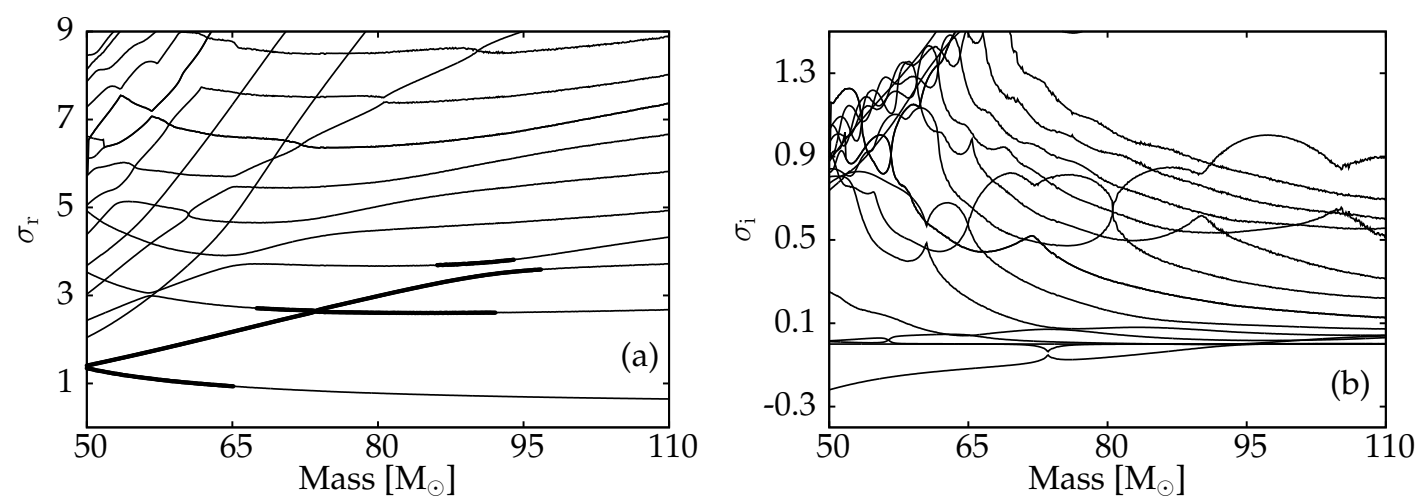

FIGURE 6.2: Modal diagram for models of $\zeta$ Pup with enhanced He abundance $(Y=0.58$ and $Z=0.02)$. Real (a) and imaginary (b) parts of the eigenfrequencies normalized by the inverse of the global free fall time are given as a function of mass. Negative imaginary parts in (b) and thick lines in (a) correspond to unstable modes.
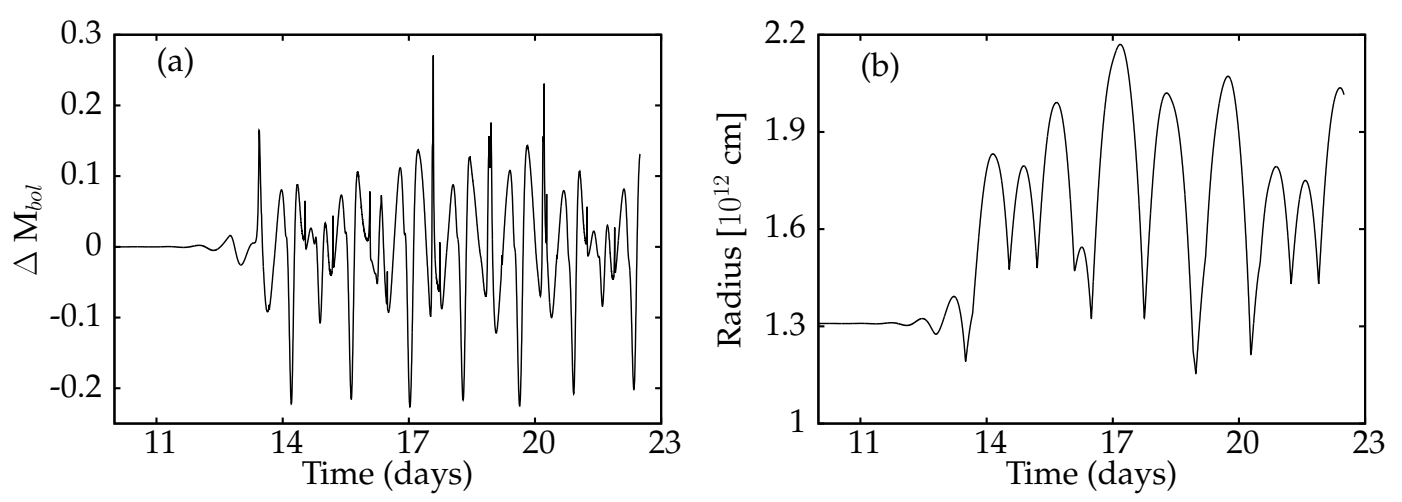

FIGURE 6.3: Nonlinear evolution of instabilities of a model for $\zeta$ Pup with $\mathrm{M}=44 \mathrm{M}_{\odot}$. The variation of the bolometric luminosity (a) and the radius (b) is given as a function of time. Note the finite amplitude period of $\approx 1.4$ days in $(\mathrm{a})$.

and confirmed by recent BRITE - satellite observations (Hubrig et al., 2016). Observations with BRITE - satellites (Baade et al., 2016; Pigulski et al., 2016; Weiss et al., 2014, 2016) provide a unique opportunity to test predictions of pulsation theory for massive stars thus contributing to our understanding of variabilities and mass loss in $\mathrm{O}$ - and B-type supergiants.

Another problem to be addressed in the future concerns the loss of resolution and accuracy caused by mass loss. Together with the mass, grid points are lost from the system since the numerical scheme adopted is based on the Lagrangian approach. To avoid resolution and accuracy problems the Lagrangian grid needs to be reconstructed. Simultaneously the conservativity of the scheme has to be preserved. This task is not trivial and has not been solved so far. Finally, it would be highly desirable to be able to follow the evolution of nonradial instabilities into the nonlinear regime. However, an appropriate 3D numerical scheme satisfying the necessary conservativity requirements is not yet available and the development of a 3D conservative scheme would be an ambitious project for the future. A conservative formulation of gravity in 3D (Glatzel \& Chernigovski, 2016) may be regarded as a first step in this direction. 


\section{Bibliography}

Aerts C., Christensen-Dalsgaard J., Kurtz D. W., 2010a, Asteroseismology

Aerts C., et al., 2010b, A\&A, 513, L11

Appenzeller I., 1970, A\&A, 9, 216

Baade D., et al., 2016, A\&A, 588, A56

Baker N., Kippenhahn R., 1962, ZAp, 54, 114

Baker N., Kippenhahn R., 1965, ApJ, 142, 868

Baraffe I., Heger A., Woosley S. E., 2001, ApJ, 550, 890

Barkana R., Loeb A., 2001, Phys. Rep., 349, 125

Barlow M. J., Cohen M., 1977, ApJ, 213, 737

Böhm-Vitense E., 1958, ZAp, 46, 108

Cantiello M., et al., 2009, A\&A, 499, 279

Cassisi S., Potekhin A. Y., Pietrinferni A., Catelan M., Salaris M., 2007, ApJ, 661, 1094

Castor J. I., 1971, ApJ, 166, 109

Chernigovski S., Grott M., Glatzel W., 2004, MNRAS, 348, 192

Christensen-Dalsgaard J., 2014, Lecture Notes on Stellar Oscillations

Ciardi B., Ferrara A., 2005, Space Sci. Rev., 116, 625

Cowling T. G., 1941, MNRAS, 101, 367

Cox J. P., 1955, ApJ, 122, 286

Cox J. P., 1980, Theory of stellar pulsation

Cox J. P., Wheeler J. C., Hansen C. J., King D. S., Cox A. N., Hodson S. W., 1980, Space Sci. Rev., 27, 529

Crowther P. A., Lennon D. J., Walborn N. R., 2006, A\&A, 446, 279

Crowther P. A., Schnurr O., Hirschi R., Yusof N., Parker R. J., Goodwin S. P., Kassim H. A., 2010, MNRAS, 408, 731

Crowther P. A., et al., 2016, MNRAS, 458, 624

Cucchiara A., et al., 2011, ApJ, 736, 7

Dantona F., Mazzitelli I., 1985, ApJ, 296, 502 
Davidson K., 1987, ApJ, 317, 760

Deller J., 2009, Bachelor thesis, Institute for Astrophysics, University of Goettingen

Dupret M.-A., Grigahcène A., Garrido R., Gabriel M., Scuflaire R., 2005, A\&A, 435, 927

Dziembowski W. A., Pamiatnykh A. A., 1993, MNRAS, 262, 204

Eddington A. S., 1926, The Internal Constitution of the Stars

Epstein I., 1950, ApJ, 112, 6

Fadeyev Y. A., 2011, Astronomy Letters, 37, 11

Figer D. F., 2005, Nature, 434, 192

Gautschy A., 1992, A\&A, 260, 175

Gautschy A., 1993, MNRAS, 265, 340

Gautschy A., 1997, Vistas in Astronomy, 41, 95

Gautschy A., Glatzel W., 1990a, MNRAS, 245, 154

Gautschy A., Glatzel W., 1990b, MNRAS, 245, 597

Gies D. R., Lambert D. L., 1992, ApJ, 387, 673

Glatzel W., 1994, MNRAS, 271, 66

Glatzel W., 1998, in Bradley P. A., Guzik J. A., eds, Astronomical Society of the Pacific Conference Series Vol. 135, A Half Century of Stellar Pulsation Interpretation. p. 89

Glatzel W., 2001, in Schielicke R. E., ed., Reviews in Modern Astronomy Vol. 14, Reviews in Modern Astronomy. p. 245

Glatzel W., 2009, Communications in Asteroseismology, 158, 252

Glatzel W., Chernigovski S., 2016, MNRAS, 457, 1190

Glatzel W., Gautschy A., 1992, MNRAS, 256, 209

Glatzel W., Kaltschmidt H. O., 2002, MNRAS, 337, 743

Glatzel W., Kiriakidis M., 1993a, MNRAS, 262, 85

Glatzel W., Kiriakidis M., 1993b, MNRAS, 263, 375

Glatzel W., Mehren S., 1996, MNRAS, 283, 339

Glatzel W., Kiriakidis M., Fricke K. J., 1993, MNRAS, 262, L7

Glatzel W., Kiriakidis M., Chernigovskij S., Fricke K. J., 1999, MNRAS, 303, 116

Goodman J., White C. J., 2016, MNRAS, 456, 525

Goodricke J., Bayer J., 1786, Philosophical Transactions of the Royal Society of London Series I, 76, 48

Grigahcène A., Dupret M.-A., Gabriel M., Garrido R., Scuflaire R., 2005, A\&A, 434, 1055 
Grott M., 2003, PhD thesis, Institute for Astrophysics, University of Goettingen

Grott M., Chernigovski S., Glatzel W., 2005, MNRAS, 360, 1532

Haehnelt M. G., Madau P., Kudritzki R., Haardt F., 2001, ApJ, 549, L151

Hilker M., 2009, Bachelor thesis, Institute for Astrophysics, University of Goettingen

Houdek G., Dupret M.-A., 2015, Living Reviews in Solar Physics, 12

Howarth I. D., Stevens I. R., 2014, MNRAS, 445, 2878

Hubrig S., Kholtygin A., Ilyin I., Schöller M., Oskinova L. M., 2016, preprint, (arXiv:1604.01068)

Humphreys R. M., Davidson K., 1979, ApJ, 232, 409

Humphreys R. M., Davidson K., 1984, Science, 223, 243

Iben Jr. I., 2013, Stellar Evolution Physics, Volume 1: Physical Processes in Stellar Interiors

Iglesias C. A., Rogers F. J., 1996, ApJ, 464, 943

Jeffery C. S., Saio H., 2016, MNRAS, 458, 1352

Jurkić T., Sarta Deković M., Dominis Prester D., Kotnik-Karuza D., 2011, Ap\&SS, 335, 113

Kambe E., Ando H., Hirata R., 1993, A\&A, 273, 435

Kippenhahn R., Weigert A., Weiss A., 2012, Stellar Structure and Evolution, doi:10.1007/978-3-642-30304-3.

Kiriakidis M., El Eid M. F., Glatzel W., 1992, MNRAS, 255, 1P

Kiriakidis M., Fricke K. J., Glatzel W., 1993, MNRAS, 264, 50

Kiriakidis M., Glatzel W., Fricke K. J., 1996, MNRAS, 281, 406

Koen C., Eyer L., 2002, MNRAS, 331, 45

Kraus M., et al., 2015, A\&A, 581, A75

Lamers H. J. G. L. M., 1986, in De Loore C. W. H., Willis A. J., Laskarides P., eds, IAU Symposium Vol. 116, Luminous Stars and Associations in Galaxies. pp 157-178

Lamers H. J. G. L. M., Cassinelli J. P., 1999, Introduction to Stellar Winds

Leavitt H. S., 1908, Annals of Harvard College Observatory, 60, 87

Ledoux P., 1941, ApJ, 94, 537

Lefèvre L., Marchenko S. V., Moffat A. F. J., Acker A., 2009, A\&A, 507, 1141

Li Y., 2000, ApJ, 538, 346

Maeder A., 1980, A\&A, 90, 311 
Maharramov Y. M., 2013, Astronomy Reports, 57, 303

Markova N., Puls J., 2008, A\&A, 478, 823

Markova N., Puls J., Scuderi S., Markov H., 2005, A\&A, 440, 1133

Matteucci F., ed. 2008, 391 IAU Symposium Vol. 250

Mendoza C., et al., 2007, MNRAS, 378, 1031

Neilson H. R., 2014, in Guzik J. A., Chaplin W. J., Handler G., Pigulski A., eds, IAU Symposium Vol. 301, IAU Symposium. pp 205-212 (arXiv:1309.4115), doi:10.1017/S1743921313014336

Noh W. F., 1987, Journal of Computational Physics, 72, 78

Nomoto K., Kobayashi C., Tominaga N., 2013, ARA\&A, 51, 457

Pamyatnykh A. A., 1999, Acta Astron., 49, 119

Papaloizou J. C. B., 1973a, MNRAS, 162, 143

Papaloizou J. C. B., 1973b, MNRAS, 162, 169

Paxton B., Bildsten L., Dotter A., Herwig F., Lesaffre P., Timmes F., 2011, ApJS, 192, 3

Paxton B., et al., 2013, ApJS, 208, 4

Paxton B., et al., 2015, ApJS, 220, 15

Percy J. R., Welch D. L., 1983, PASP, 95, 491

Pigulski A., et al., 2016, A\&A, 588, A55

Prinja R. K., Markova N., Scuderi S., Markov H., 2006, A\&A, 457, 987

Puls J., Vink J. S., Najarro F., 2008, A\&A Rev., 16, 209

Ritter A., 1879, Wiedemanns Annalen VIII, p. 173

Rogers F. J., Iglesias C. A., 1992, ApJS, 79, 507

Rogers F. J., Swenson F. J., Iglesias C. A., 1996, ApJ, 456, 902

Rufener F., Bartholdi P., 1982, A\&AS, 48, 503

Saio H., 2011, MNRAS, 412, 1814

Saio H., Jeffery C. S., 1988, ApJ, 328, 714

Saio H., Wheeler J. C., 1985, ApJ, 295, 38

Saio H., Wheeler J. C., Cox J. P., 1984, ApJ, 281, 318

Saio H., Baker N. H., Gautschy A., 1998, MNRAS, 294, 622

Salaris M., Cassisi S., 2006, Evolution of Stars and Stellar Populations

Schwarzschild M., Härm R., 1959, ApJ, 129, 637 
Scott M. R., 1973, Journal of Computational Physics, 12, 334

Searle S. C., Prinja R. K., Massa D., Ryans R., 2008, A\&A, 481, 777

Shapley H., 1914, ApJ, 40, 448

Shiode J. H., Quataert E., Arras P., 2012, MNRAS, 423, 3397

Smith N., 2014, ARA\&A, 52, 487

Smolec R., 2009, PhD thesis, Nicolaus Copernicus Astronomical Center, Warsaw, Poland

Sonoi T., Shibahashi H., 2014, PASJ, 66, 69

Sonoi T., Umeda H., 2012, MNRAS, 421, L34

Stothers R. B., 1992, ApJ, 392, 706

Stothers R. B., Chin C.-W., 1993, ApJ, 412, 294

Talbot Jr. R. J., 1971a, ApJ, 163, 17

Talbot Jr. R. J., 1971b, ApJ, 165, 121

Tanvir N. R., et al., 2009, Nature, 461, 1254

Underhill A. B., 1969, A\&A, 1, 494

Unno W., Osaki Y., Ando H., Shibahashi H., 1979, Nonradial oscillations of stars

Vink J. S., de Koter A., Lamers H. J. G. L. M., 2001, A\&A, 369, 574

Von Neumann J., Richtmyer R. D., 1950, Journal of Applied Physics, 21, 232

Weidner C., Kroupa P., 2004, MNRAS, 348, 187

Weiss W. W., et al., 2014, PASP, 126, 573

Weiss W. W., et al., 2016, A\&A, 588, A54

Wood P. R., 1976, MNRAS, 174, 531

Wood P. R., Olivier E. A., 2014, MNRAS, 440, 2576

Yadav A. P., Glatzel W., 2016, MNRAS, 457, 4330

Yadav A. P., Glatzel W., 2017, MNRAS, 465, 234

Zhevakin S. A., 1963, ARA\&A, 1, 367

Ziebarth K., 1970, ApJ, 162, 947

de Jager C., 1980, Geophysics and Astrophysics Monographs, 19

de Jager C., 1984, A\&A, 138, 246

van Genderen A. M., 1989, A\&A, 208, 135 



\title{
Curriculum vitae
}

\author{
Name : Abhay Pratap Yadav \\ Date of Birth : $\quad 01^{\text {st }}$ September 1989 \\ Place of Birth : Ghazipur (India) \\ Nationality : Indian
}

\section{Education}

10/2013 - 07/2016 I PhD studies at Institute for Astrophysics,

University of Goettingen, Germany

Thesis title: "On the stability of massive stars"

09/2011 - 09/2013 I AstroMundus Joint Master of Science Degree in Astrophysics,

Coordinated by University of Innsbruck, Austria

Thesis title 'Study of chromospheric activity of Solar-type stars'

07/2008 - 06/2010 I Master of Science in Physics

Rani Durgavati Vishwavidyalaya Jabalpur, India

07/2005 - 06/2008 I Bachelor of Science with Physics, Mathematics and Chemistry

Rani Durgavati Vishwavidyalaya Jabalpur, India

\section{Publications}

1. Yadav A.P. and Glatzel W., "Stability analysis, non-linear pulsations and mass loss of models for 55 Cygni (HD 198478)", MNRAS, 457, 4330-4339, 2016.

2. Yadav A.P. and Glatzel W., "Strange mode instability driven finite amplitude pulsations and mass-loss in models of massive zero-age main-sequence stars", MNRAS, 465, 234-241, 2017.

\section{Conference Contributions}

- Annual Meeting of German Astronomical Society, Kiel, September 14-18, 2015

Poster: "Instabilities and pulsations in models of 55 Cygni" 